\title{
Cepheid Calibrations from the Hubble Space Telescopeof the Luminosity of Two Recent Type la Supernovae and a Redetermination of the Hubble Constant
}

\section{Citation}

Riess, Adam G., Weidong Li, Peter B. Stetson, Alexei V. Filippenko, Saurabh Jha, Robert P. Kirshner, Peter M. Challis, Peter M. Garnavich, and Ryan Chornock. 2005. “Cepheid Calibrations from the Hubble Space Telescopeof the Luminosity of Two Recent Type la Supernovae and a Redetermination of the Hubble Constant." The Astrophysical Journal 627 (2): 579-607. https:// doi.org/10.1086/430497.

\section{Permanent link}

http://nrs.harvard.edu/urn-3:HUL.InstRepos:41399914

\section{Terms of Use}

This article was downloaded from Harvard University's DASH repository, and is made available under the terms and conditions applicable to Other Posted Material, as set forth at http:// nrs.harvard.edu/urn-3:HUL.InstRepos:dash.current.terms-of-use\#LAA

\section{Share Your Story}

The Harvard community has made this article openly available.

Please share how this access benefits you. Submit a story.

Accessibility 


\title{
CEPHEID CALIBRATIONS FROM THE HUBBLE SPACE TELESCOPE OF THE LUMINOSITY OF TWO RECENT TYPE Ia SUPERNOVAE AND A REDETERMINATION OF THE HUBBLE CONSTANT ${ }^{1}$
}

\author{
Adam G. Riess, ${ }^{2}$ Weidong Li, ${ }^{3}$ Peter B. Stetson, ${ }^{4}$ Alexei V. Filippenko, ${ }^{3}$ Saurabh Jha, ${ }^{3}$ \\ Robert P. Kirshner, ${ }^{5}$ Peter M. Challis, ${ }^{5}$ Peter M. Garnavich, ${ }^{6}$ and Ryan Chornock ${ }^{3}$ \\ Received 2005 January 28; accepted 2005 March 9
}

\begin{abstract}
We report observations of two nearby Type Ia supernovae (SNe Ia) for which observations of Cepheid variables in the host galaxies have been obtained with the Hubble Space Telescope: SN 1994ae in NGC 3370 and SN $1998 \mathrm{aq}$ in NGC 3982. For NCG 3370, we used the Advanced Camera for Surveys to observe 64 Cepheids that yield a distance of $29 \mathrm{Mpc}$, the farthest direct measurement of Cepheids. We have measured emission lines from H II regions in both host galaxies that provide metallicity-dependent corrections to their period-luminosity relations. These two SNe Ia double the sample of "ideal" luminosity calibrators: objects with well-observed and wellcalibrated light curves of typical shape and with low reddening. By comparing them to all similarly well-measured $\mathrm{SNe}$ Ia in the Hubble flow, we find that $H_{0}=73 \pm 4$ (statistical) \pm 5 (systematic) $\mathrm{km} \mathrm{s}^{-1} \mathrm{Mpc}^{-1}$. A detailed analysis demonstrates that most of the past disagreement over the value of $H_{0}$ as determined from SNe Ia is abated by the replacement of past, problematic data by more accurate and precise, modern data.
\end{abstract}

Subject headings: cosmology: observations — distance scale — galaxies: distances and redshifts supernovae: general

Online material: machine-readable tables

\section{INTRODUCTION}

Type Ia supernovae (SNe Ia) are the most precise distance indicators known for measuring the expansion rate of the universe. Their extreme and relatively uniform luminosities provide the means to measure accurate and precise distances across a significant fraction of the visible universe (for a review see Leibundgut 2001) and gave the first evidence of its accelerating expansion (Riess et al. 1998; Perlmutter et al. 1999). Methods that utilize the relationship between SN Ia light-curve shape and luminosity (Phillips 1993; Hamuy et al. 1995, 1996a; Riess et al. 1995, 1996a; Perlmutter et al. 1997) and supernova color to measure absorption by dust (Riess et al. 1996b; Phillips et al. 1999) yield distances with relative precision approaching $7 \%$ when applied to modern photometry. The tightest Hubble diagram is provided by SNe Ia (see Fig. 1). The $\sim 90$ high-quality SN Ia light curves currently published in the range $0.01<z<0.1$ establish the relative expansion rate to an unprecedented uncertainty of less than $1 \%$. One-quarter of these $\mathrm{SNe}$ Ia are from the Calán/Tololo survey (Hamuy et al. 1996b), and the rest are from the CfA Survey I (Riess et al. 1999a) and the CfA Survey II (Jha et al. 2005).

\footnotetext{
1 Based on observations with the NASA/ESA Hubble Space Telescope, obtained at the Space Telescope Science Institute, which is operated by AURA, Inc., under NASA contract NAS 5-26555.

2 Space Telescope Science Institute, 3700 San Martin Drive, Baltimore, MD 21218; ariess@stsci.edu.

3 Department of Astronomy, University of California, 601 Campbell Hall, Berkeley, CA 94720-3411; weidong@astro.berkeley.edu, alex@astro.berkeley .edu, sjha@astro.berkeley.edu,rchornock@astro.berkeley.edu.

4 Herzberg Institute of Astrophysics, National Research Council, 5071 West Saanich Road, Victoria, BC V9E 2E7, Canada; peter.stetson@nrc-cnrc.gc.ca.

5 Harvard-Smithsonian Center for Astrophysics, 60 Garden Street, Cambridge, MA 02138; kirshner@cfa.harvard.edu.

6 Department of Physics, University of Notre Dame, 225 Nieuwland Science Hall, Notre Dame, IN 46556-5670; pgarnavi@miranda.phys.nd.edu.
}

A calibration of the peak luminosity of SNe Ia, while not necessary for past measurements of changes in the expansion rate, is needed to fix the distance scale of the universe by determining the Hubble constant. Many other quantities of interest in cosmology and astrophysics directly depend on the value of the Hubble constant, including the expansion age of the universe, the size of the visible universe, the closure density, and the baryon density, to name a few. An accurate determination of $H_{0}$ also enables the extraction of the mass density and energy density from the measurement of the distance to the last scattering surface, for example, by the Wilkinson Microwave Anisotropy Probe (Bennett et al. 2003; Spergel et al. 2003).

An accurate and precise measurement of the Hubble constant was one of the original motivations for the construction of the Hubble Space Telescope (HST), and two teams ambitiously tackled the challenge of determining $H_{0}$ by analyzing $H S T$ observations of Cepheid variables (beginning even before the replacement of WF/PC with WFPC2).

The Type Ia Supernova HST Calibration Program, led by A. Saha, G. Tammann, and A. Sandage (hereafter STS; see Saha et al. 1997, 2001 and references therein), has produced calibrations of the luminosity of eight individual SNe Ia using $H S T$ observations of Cepheid variables in their host galaxies. However, the limited volume within which $H S T$ with WFPC2 can resolve Cepheids $(R \approx 20 \mathrm{Mpc})$ severely limits the quality of supernova data to calibrate. Half of the SNe Ia calibrated by STS were discovered before the advent of CCDs (see Table 1). The light curves of SN 1895B, SN 1937C, SN 1960F, and SN 1974G were obtained with photographic emulsions and show internal dispersions of $\sim 0.2 \mathrm{mag}$. An accurate accounting of the background light is difficult to achieve and frequently unreliable. Inaccurate subtraction of background light from such data can result in systematic errors in peak brightness and lightcurve shape, as described by Boisseau \& Wheeler (1991). In addition, the nonstellar spectrum of SNe Ia makes it difficult 


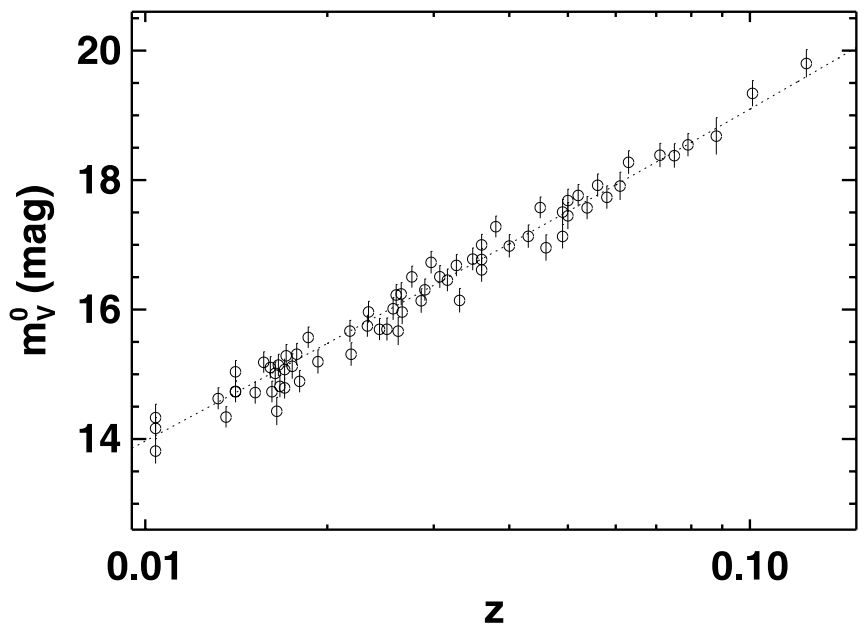

FIG. 1.-MLCS2k2 SN Ia Hubble diagram using the Gold sample from Riess et al. (2004). Parameter $m_{V}^{0}$ (rest) is the peak apparent magnitude scaled to the luminosity of the fiducial $(\Delta=0) \mathrm{SN}$ Ia, corrected for reddening, and transformed to the rest-frame $V$ band. Conversion from apparent magnitudes to distance requires knowledge of the distance scale or peak luminosity of the fiducial SN Ia. Overplotted is the best fit for a flat cosmology $\left(\Omega_{M}=0.29\right.$, $\left.\Omega_{\Lambda}=0.71\right)$.

to accurately convert photographic magnitudes to standard passbands.

Dramatic progress in the cosmological utility of $\mathrm{SNe}$ Ia has been made possible by improvements in the quality of their photometric data in the last two decades. It is time for the pursuit of Hubble's constant via SNe Ia to overcome the problems inherent in predigital data and benefit from superior calibrations.

Additional liens against past calibrators include objects with high reddening leading to large and thus more uncertain extinction (e.g., $A_{V} \geq 1 \mathrm{mag}$ for SN 1989B and SN 1998bu) and objects with atypical light-curve shapes leading to relatively large and thus more method-dependent luminosity corrections (e.g., SN 1991T and SN 1999by). The use of such objects risks contaminating the fiducial SN Ia luminosity determined from the small sample of calibrators. (This danger is not an issue for the Hubble-flow set of SNe Ia, which is large enough to overcome a few inaccurate objects.)
Only 2 of the 11 calibrated SNe Ia (SN 1981B and SN 1990N) are ideal. These have been observed before maximum light, through low interstellar extinction $\left(A_{V}<0.5 \mathrm{mag}\right)$, with the same instruments (CCDs and the Johnson/Cousins passbands) as the SNe Ia in the Hubble flow from the Calán/Tololo and CfA Surveys, and have typical light-curve shapes. Interestingly, the average magnitude of these two SNe Ia is 0.14 mag fainter than the average of the other 9 less optimal calibrators (Saha et al. 2001). Clearly the best course of action will be to increase the small sample of such calibrators.

Another impediment to an accurate resolution of the Hubble constant has been disagreement over the analysis of Cepheid data. A subset of the HST Key Project (hereafter referred to as "SKP") has reached significantly different conclusions about the value of $H_{0}$ from precisely the same data utilized by the STS group (Gibson et al. 2000). By reanalyzing the Cepheid data of STS, the SKP concludes that each of the SN Ia calibrators is intrinsically $0.2-0.3 \mathrm{mag}$ fainter (even when both groups utilize the same 18.50 mag distance modulus of the Large Magellanic Cloud [LMC] and agree on the measured fluxes of the HST Cepheids). We believe that an independent look at these differences is warranted. We find that the origin of at least some of the differences in interpretation rests in pernicious anomalies in WFPC2 that can now be circumvented by the new Advanced Camera for Surveys (ACS) on HST.

The other improvement offered by ACS is that HST can now well measure the light curves of Cepheids in an economical amount of observing time at a distance of up to $\sim 30 \mathrm{Mpc}$. This increased range provides an opportunity to add to the small calibrated sample of ideal SNe Ia. In $\S 2$ we present observations of Cepheid variables in NGC 3370 obtained with ACS on HST in a 55 day campaign during Cycle 11. NGC 3370 was the host of SN 1994ae, a spectroscopically normal (Filippenko 1997; Branch et al. 1993) SN Ia observed through UBVRI filters with modern CCDs beginning 12 days before maximum brightness (Riess et al. 1999b). The SN has $\lesssim 0.25$ mag of visual extinction from its host, reducing uncertainties from unknown extinction laws. In $\S 3$ we present a careful recalibration of the light curve using additional photometric calibrations of the field and galaxy template subtraction to remove contamination of the SN photometry. We also present the first photometric record of SN 1998aq, a similarly well-observed prototypical SN Ia with low reddening.

TABLE 1

SN Ia Light Curves with hST Cepheid Calibration

\begin{tabular}{|c|c|c|c|c|c|}
\hline SN Ia & $\begin{array}{c}\text { CCD } \\
\text { Photometry? }\end{array}$ & $\begin{array}{c}\text { Low } \\
\text { Reddening? }^{\text {a }}\end{array}$ & $\begin{array}{l}\text { Observed before } \\
\text { Maximum? }\end{array}$ & Normal? & Ideal? \\
\hline $1895 \mathrm{~B}^{\mathrm{b}}$ & No & Unknown & No & Unknown & No \\
\hline $1937 C^{\mathrm{b}}$ & No & Yes & Yes & Yes & No \\
\hline $1960 \mathrm{~F}^{\mathrm{b}}$ & No & No & Yes & $?$ & No \\
\hline $1972 E^{\mathrm{b}} \ldots \ldots \ldots \ldots \ldots \ldots$ & No & Yes & No & Yes & No \\
\hline 1974G.............................. & No & No & Yes & $?$ & No \\
\hline 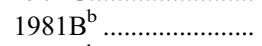 & Yes & Yes & Yes & Yes & Yes \\
\hline $1989 \mathrm{~B}^{\mathrm{b}}$. & Yes & No & Yes & Yes & No \\
\hline $1990 \mathrm{~N}^{\mathrm{b}}$ & Yes & Yes & Yes & Yes & Yes \\
\hline $1991 \mathrm{~T}^{\mathrm{b}} \ldots \ldots \ldots \ldots \ldots \ldots \ldots \ldots$ & Yes & Yes? & Yes & No & No \\
\hline $1994 \mathrm{ae}^{\mathrm{c}}$ & Yes & Yes & Yes & Yes & Yes \\
\hline $1998 \mathrm{aq}^{\mathrm{c}}$ & Yes & Yes & Yes & Yes & Yes \\
\hline 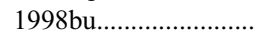 & Yes & No & Yes & Yes & No \\
\hline 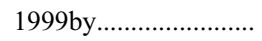 & Yes & Yes & Yes & No & No \\
\hline
\end{tabular}

\footnotetext{
${ }^{\text {a }} A_{V}<0.5$ mag.

b Calibrated by the STS collaboration.

${ }^{c}$ Calibration first presented in this paper.
} 


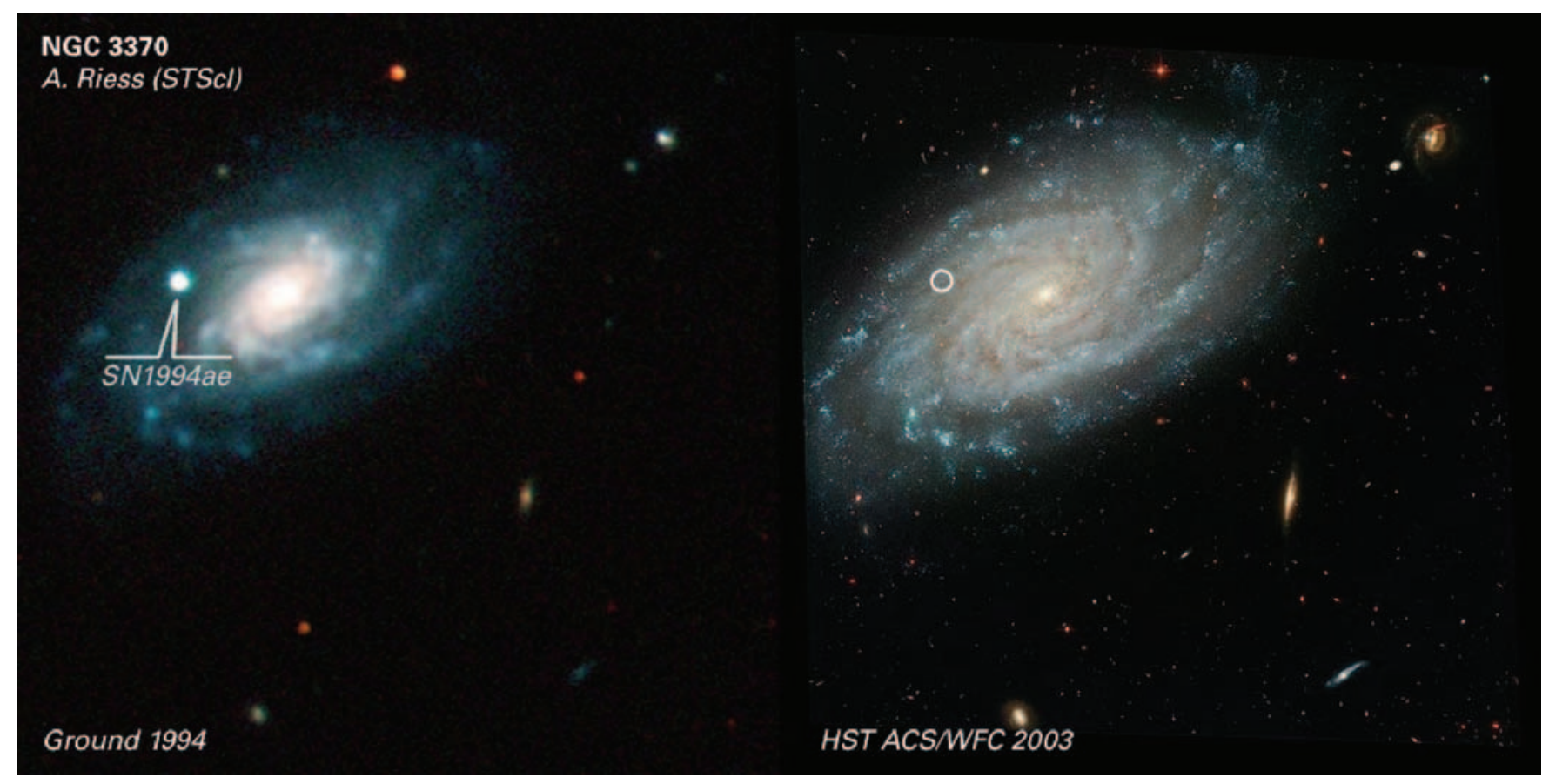

FIG. 2.-Color images of a $3^{\prime} \times 3^{\prime}$ region of the field of NGC 3370 and the SN Ia 1994ae. The left panel was produced from $B$-, $V$-, and $I$-band images obtained at the FLWO $1.2 \mathrm{~m}$ telescope in 1994 December, when SN 1994ae was bright but well past maximum. The right panel is the same region produced from a stack of F435W, F555W, and F814W images obtained with ACS on HST in 2003

Combined with the recent Cepheid calibration of its host, NGC 3982 (Saha et al. 2001; Stetson \& Gibson 2001), we calibrate its luminosity. These two SNe Ia double the sample of ideal SNe Ia whose properties and photometric records can provide the most reliable absolute calibrations for the SN Ia Hubble diagram. In $\S 4$ we present estimates of $H_{0}$, and we discuss the analysis in $\S 5$.

\section{THE CEPHEID-CALIBRATED DISTANCE TO NGC 3370}

\subsection{Observations}

NGC 3370 is a relatively small $(3 . ' 0 \times 2.5)$, nearly face-on Sc galaxy (see Fig. 2), identified as a member of a loose group (the Lyon Group LGG 219; Garcia 1993) that includes four other spirals and one irregular. Its heliocentric recession velocity is $1280 \mathrm{~km} \mathrm{~s}^{-1}$ as measured from systemic $21 \mathrm{~cm} \mathrm{H}$ I (de Vaucouleurs et al. 1991). Based on the peak brightness of SN 1994ae ( $V=$ $13.1 \mathrm{mag}$; Riess et al. 1999b), the low reddening at maximum ( $B-V=0.1 \mathrm{mag})$, and previous calibrations of the peak luminosity $\left(M_{V}=-19.4\right.$ mag; Jha et al. 1999; Saha et al. 2001), we anticipated a distance modulus of $\sim 32.4$ mag (distance about $30 \mathrm{Mpc}$ ) when proposing for HST observations of Cepheids in this host.

Given the late-type morphology, low inclination, and estimated distance, NGC 3370 appeared to be an excellent candidate for hosting Cepheid variables whose light curves could be measured in an economical amount of time with ACS. We used exposure times of 2 orbits ( $4800 \mathrm{~s}$ ) per epoch, which was expected to give a signal-to-noise ratio $(\mathrm{S} / \mathrm{N})$ of $10-25$ for Cepheids of 20 days $<P<60$ days $(27.4 \mathrm{mag}<$ F555W $<26.1 \mathrm{mag}$ ), similar to what was obtained by the SKP and STS in most of their programs. In all, we observed for 12 epochs in $\mathrm{F} 555 \mathrm{~W}$ with power-law interval spacing over a 53 day span to reduce aliasing (Freedman et al. 1994) and five epochs in F814W to aid in the identification of Cepheids and to measure the mean dust extinction from their colors. The exposure information is contained in Table 2.
The telescope orientation and position were fixed throughout the observations to simplify the data reduction (but small shifts of $1-2$ pixels are routine for visits that use the same guide stars throughout). Imaging at each epoch was obtained at four dither positions (each of $1200 \mathrm{~s}$ duration): two positions with a small (noninteger) shift of a few pixels in the $x$ - and $y$-directions repeated after a large shift of 60 pixels in the $y$-direction to cover the ACS chip gap. (The reason for filling in the gap was to produce an aesthetically pleasing image suitable for a Hubble Heritage Program press release.) The orientation and position were chosen to contain a few external (to NGC 3370) bright sources in the ACS field that were also visible in the original monitoring data of SN 1994ae (Riess et al. 1999b) from the $1.2 \mathrm{~m}$ telescope at the Fred Lawrence Whipple Observatory (FLWO), as seen in Figure 2. (A similar view of SN 1998aq and HST images of its host are shown in Fig. 3.) Matching these sources provides the means to register the HST images to the groundbased SN follow-up data and allows us to search for a possible light echo or late-time visibility of SN 1994ae with high astrometric precision (although ultimately neither was detected to the limit of the frames, F555W $\approx 29.0 \mathrm{mag}$ ).

After acquisition, all images were processed using up-todate reference files and the CALACS pipeline in the STSDAS package in IRAF. ${ }^{7}$ This procedure includes "standard" rectifications for the camera gain, overscan, spatial bias, dark current, and flat fielding. Due to the significant geometric distortion of the ACS WFC (the cost of minimizing reflections), we applied the "drizzle" algorithm (Fruchter \& Hook 1997) in the Multidrizzle software package (A. Koekemoer et al. 2005, in preparation). Because ACS WFC images are undersampled at wavelengths shortward of 11,000 $\AA$, a better sampled and more precise point-spread function (PSF) can be obtained by "drizzling"

${ }^{7}$ IRAF is distributed by the National Optical Astronomy Observatory, which is operated by the Association of Universities for Research in Astronomy, Inc., under cooperative agreement with the National Science Foundation. 
TABLE 2

HST OBSERVATIONS OF NGC 3370

\begin{tabular}{|c|c|c|c|c|}
\hline Epoch & $\begin{array}{l}\text { HST Archive } \\
\text { Root Name }\end{array}$ & UT Date & $\mathrm{MJD}^{\mathrm{a}}$ at Start & $\begin{array}{c}\text { Exposure Time } \\
\text { (s) }\end{array}$ \\
\hline \multicolumn{5}{|c|}{ F555W } \\
\hline $1 \ldots \ldots \ldots$ & $\mathrm{j} 8 \mathrm{~d} 211 \mathrm{dzq}$ & 2003 Mar 31 & 52729.51097 & 4800.0 \\
\hline $2 \ldots \ldots \ldots$ & $\mathrm{j} 8 \mathrm{~d} 212 \mathrm{pdq}$ & 2003 Apr 08 & 52737.58257 & 4800 \\
\hline $3 \ldots \ldots$ & j8d213giq & 2003 Apr 16 & 52745.38616 & 4800 \\
\hline $4 \ldots$. & $\mathrm{j} 8 \mathrm{~d} 214 \mathrm{f} 5 \mathrm{q}$ & 2003 Apr 21 & 52750.92149 & 4800 \\
\hline $5 \ldots \ldots \ldots$ & j8d215neq & 2003 Apr 27 & 52756.12557 & 4800 \\
\hline $6 \ldots \ldots \ldots$ & $\mathrm{j} 8 \mathrm{~d} 216 \mathrm{p} 4 \mathrm{q}$ & 2003 Apr 29 & 52758.05614 & 4800 \\
\hline $7 \ldots \ldots \ldots$ & $\mathrm{j} 8 \mathrm{~d} 217 \mathrm{a} 6 \mathrm{q}$ & 2003 May 01 & 52760.12780 & 4800 \\
\hline $8 \ldots \ldots \ldots$ & $\mathrm{j} 8 \mathrm{~d} 218 \mathrm{~m} 8 \mathrm{q}$ & 2003 May 03 & 52762.05838 & 4800 \\
\hline $9 \ldots \ldots \ldots$ & $\mathrm{j} 8 \mathrm{~d} 219 \mathrm{v} 7 \mathrm{q}$ & 2003 May 07 & 52766.13153 & 4800 \\
\hline $10 \ldots \ldots \ldots$ & $\mathrm{j} 8 \mathrm{~d} 21 \mathrm{amgq}$ & 2003 May 09 & 52768.41565 & 4800 \\
\hline $11 \ldots \ldots \ldots$ & $\mathrm{j} 8 \mathrm{~d} 21 \mathrm{bbqq}$ & 2003 May 15 & 52774.40382 & 4800 \\
\hline $12 \ldots \ldots \ldots$ & j8d21capq & 2003 May 22 & 52781.41830 & 4800 \\
\hline \multicolumn{5}{|c|}{ F814W } \\
\hline $1 \ldots \ldots \ldots$ & $\mathrm{j} 8 \mathrm{~d} 211$ etq & 2003 Mar 31 & 52729.64275 & 4800 \\
\hline $3 \ldots \ldots \ldots$ & j8d213gzq & 2003 Apr 16 & 52745.51695 & 4800 \\
\hline $7 \ldots \ldots \ldots$ & j8d217avq & 2003 May 01 & 52760.33845 & 4800 \\
\hline $10 \ldots \ldots \ldots$ & j8d21amyq & 2003 May 09 & 52768.54504 & 4800 \\
\hline $12 \ldots \ldots \ldots$ & j8d21cauq & 2003 May 22 & 52781.46974 & 4800 \\
\hline
\end{tabular}

${ }^{\text {a }}$ MJD is the Julian Date minus 2,400,000.

(i.e., resampling and combining) the images at a pixel scale finer than the physical detector pixels of $0 . " 05$ pixel $^{-1}$. However, full resampling can only be realized with well-dithered images. The relative size of the dither was measured for each frame using source catalogs. The images were subsequently resampled to $0^{\prime \prime} 033$ pixel $^{-1}$.
ACS PSFs were constructed in the usual way from uncrowded frames of 47 Tucanae (NGC 104) obtained by an ACS calibration program (GO 9648). Since only one (drizzled) image of 47 Tuc was used to define the PSF for each filter, the normal DAOPHOT "PSF" command was used, rather than the more powerful program MULTIPSF (Stetson 1993). Approximately 250 stars well distributed across the 47 Tuc field were used to define PSFs with quadratic spatial variation for the three filters. The derived PSFs did a good job of subtracting the stars from the images, so we infer that the spatial variations in the (geometrically rectified) images are well represented by our model.

Since the drizzled images employed here were already largely free of cosmic-ray events, we employed a reduction process similar to that used for reducing ground-based data. Catalogs of stellar-appearing objects in 47 Tuc and NGC 3370 were produced by the "FIND" command in DAOPHOT, and initial magnitude estimates and local sky brightness values were obtained with the "PHOT" command. At this point the aforementioned PSFs were obtained from the images of 47 Tuc by an iterated application of the "PSF" command and the program ALLSTAR (see, e.g., Stetson 1987). These PSFs were then employed to analyze the NGC 3370 images with ALLSTAR to produce improved magnitude and sky brightness estimates for the detections in the individual images. This process normally results in the rejection of some poor-quality and evidently nonstellar detections. These refined individual star lists were then intercompared and merged by the programs DAOMATCH and DAOMASTER, a process that produces both a master list of unresolved sources in the field and a set of geometric transformations relating the coordinate systems of the individual images to a common reference frame. These are the raw inputs to the program ALLFRAME, which enforces self-consistent positions for the detected objects as it produces improved estimates of the individual-epoch magnitudes and geometric transformations. Then the original and

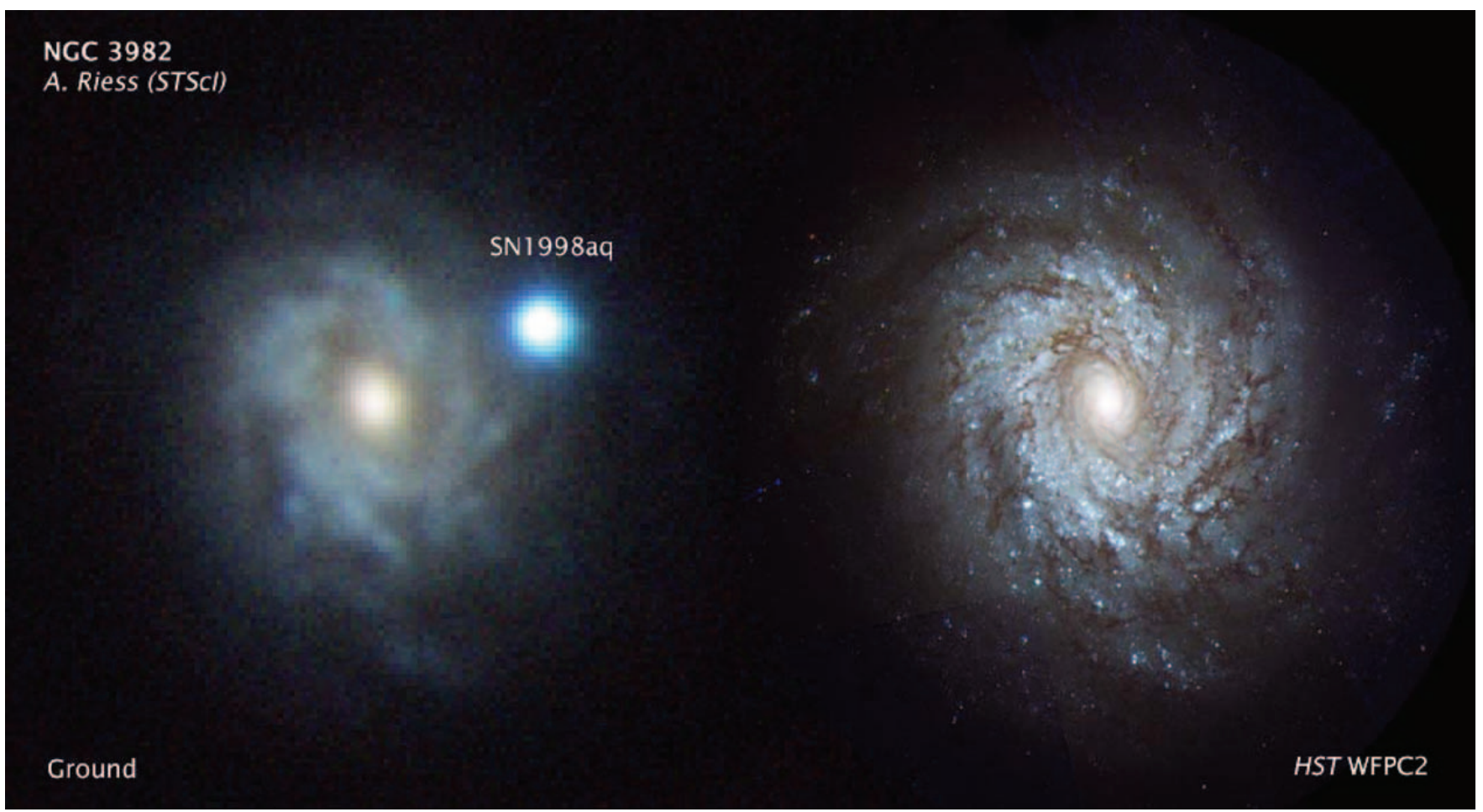

Fig. 3. - Color images of a $3^{\prime} \times 3^{\prime}$ region of the field of NGC 3982 and the SN Ia 1998aq. The left panel was produced from $B$-, $V$-, and $I$-band images obtained at the FLWO $1.2 \mathrm{~m}$ telescope in 1998 when SN 1998aq was near maximum brightness. The right panel is the same region produced from a stack of F435W, F555W, and F814W images obtained with WFPC2 on HST in 2001. 
star-subtracted images were stacked and examined by eye to identify starlike images that had been missed by the automatic routines. These were added to the master list for the field, and the DAOMASTER and ALLFRAME reductions were repeated until we judged that all the stellar objects that we could see had been adequately reduced.

Photometric calibration of the sources was derived in two independent ways. One method, similar to what was done by the SKP, was to derive photometric transformations by matching the ACS data for 47 Tuc in F435W, F555W, and F814W to ground-based photometry for stars in the cluster. This photometry was derived from the same images employed by Stetson (2000) to define secondary standard stars in the cluster field. These observations were obtained on 22 nights from 12 distinct observing runs with various telescopes (Stetson 2000). Calibrated magnitudes were extracted from the master data set for the same 250 hand-selected stars as were used to define the PSFs in the ACS images of the 47 Tuc field. The median number of independent measurements of the magnitude of each star was 31 in each of $B$ and $V$ (minimum 25, maximum 32 ) and $36(30,37)$ in $I$, and the median photometric uncertainty of the derived ground-based magnitudes was $0.003 \mathrm{mag}$ in $B$, 0.002 in $V$, and 0.002 in $I$. A ground-based color-magnitude diagram for these 250 local standards is shown in Figure 4. While there is only a sprinkling of stars with extreme colors suitable for defining the color terms in the photometric transformation, the calibration should be extremely well defined for stars with the neutral colors of Cepheid variables $(B-I \approx$ $1.3-1.8 \mathrm{mag})$.

The ACS measurements of these same 250 stars implied the following transformation equations:

$$
\begin{gathered}
b_{\mathrm{ACS}}=B+\text { const }-0.091(B-V)+0.075(B-V)^{2}, \\
v_{\mathrm{ACS}}=V+\text { const }+0.018(V-I)+0.038(V-I)^{2},
\end{gathered}
$$

and

$$
i_{\mathrm{ACS}}=I+\text { const }+0.088(V-I)-0.030(V-I)^{2} .
$$

Here we do not report the quantitative values of the zero-point constant because they would not apply to ACS images drizzled differently from the way we have done; the 47 Tuc images we employed had been drizzled in exactly the same way as the NGC 3370 images, and we transferred these zero points and the color transformations. The standard deviation of the 250 individual stars about these mean relations was 0.015 mag in $B$ and $V$ and 0.026 mag in $I$, so the contribution of random calibration uncertainty to the total error budget should be very small (formally $<0.002 \mathrm{mag}$ ). Accordingly, our external errors should be dominated by any time variation in the ACS throughput and other systematics stemming from differences between the 47 Tuc images and the NGC 3370 images.

This photometric solution was applied to 51 bright, isolated stars (Table 3 ) intended to serve as local reference standards in the NGC 3370 field. This list may be used in the future to test the zero points or color dependencies of our photometry. The photometric solution was also applied to the faint stars in NGC 3370 yielding estimated stellar magnitudes in the Landolt (1992) version of the Johnson/Cousins photometric system. This is the same general procedure used by the SKP (e.g., Hill et al. 1998), in that the SKP also derived transformations of HST data to Landolt's ground-based $V_{J}, I_{C}$ system from a preliminary version

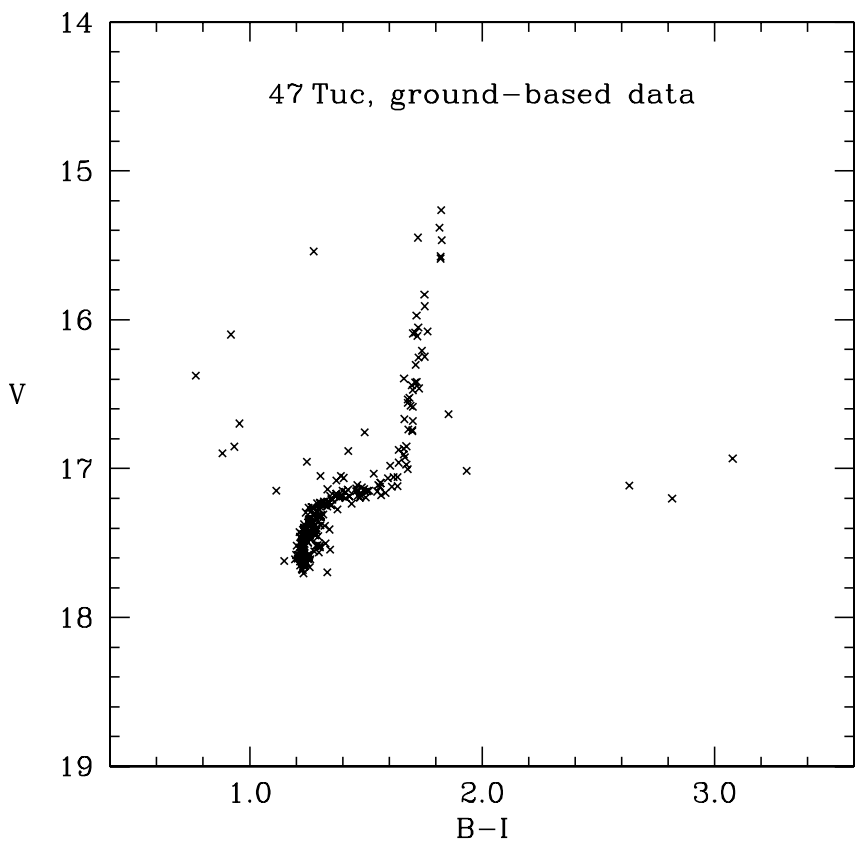

FIG. 4.- Hertzsprung-Russell diagram of 250 stars observed from the ground in the cluster 47 Tuc and used to define a photometric transformation between ACS passbands and the Johnson/Cousins system as calibrated by Landolt (1992). These stars were hand selected as those that are well detected in both the ACS images and ground-based images from Stetson (2000). Each of the ground-based points is based on $25-37$ independent measurements, with a final photometric uncertainty of 2-3 millimag.

of Stetson's homogeneous standard system. The present calibration is independent of that used by the SKP, in that theirs was based on the clusters Pal 4 and NGC 2419 and was employed, of course, for calibrating WFPC2 rather than ACS.

An independent route was to derive magnitudes for the field stars and those in NGC 3370 by utilizing the "official" photometric calibration obtained by the ACS Team and STScI (Sirianni et al. 2005). This calibration is based on an empirical measurement of the system throughput at all wavelengths using the known spectral energy distributions of five white dwarfs. A transformation from the ACS natural system to the Johnson/ Cousins system was derived by producing synthetic magnitudes from spectrophotometry of a wide range of stellar types. This method is similar to the WFPC2 calibration by Holtzman et al. (1995). We use the 51 local standards in the NGC 3370 field to compare the two methods of calibrations in $\S 2.3$.

Comparisons of photometry from the same WFPC2 images for 118 Cepheids using two popular PSF fitting tools, DoPHOT and DAOPHOT, show that these methods yield magnitudes that typically agree to within a few times $10^{-2} \mathrm{mag}$ (Gibson et al. 2000; Saha et al. 2001). Leonard et al. (2003) compared photometry obtained by DAOPHOT/ALLFRAME and HSTphot (a version of DoPhot customized by Dolphin [2000] for WFPC2) for nonvariable stars in NGC 1637 and derived a mean difference of $\Delta V=0.015 \pm 0.022 \mathrm{mag}$ and $\Delta I=0.036 \pm 0.018 \mathrm{mag}$; for our purposes such differences are negligible. Indeed, according to Saha et al. (2001), the difference in distances measured from the same data by SKP and STS lies not in the method used to fit the PSF but rather in differences in the samples of Cepheids selected and how they are treated; we thus concluded that it was not necessary to repeat measurements with DoPhot. We explore the sensitivity to the analysis parameters in $\S \S 2.2$ and 2.3. 
TABLE 3

Comparison Stars in ACS Field of NGC 3370

\begin{tabular}{|c|c|c|c|c|}
\hline ID & $x^{\mathrm{a}}$ & $y^{\mathrm{a}}$ & $\langle V\rangle^{\mathrm{b}}$ & $\langle I\rangle^{\mathrm{b}}$ \\
\hline 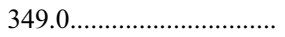 & 326.25 & 202.78 & $22.935 \pm 0.006$ & $22.137 \pm 0.010$ \\
\hline 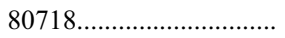 & 929.95 & 5355.2 & $22.416 \pm 0.006$ & $20.248 \pm 0.008$ \\
\hline 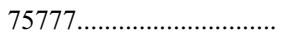 & 954.68 & 4014.5 & $24.411 \pm 0.014$ & $23.762 \pm 0.006$ \\
\hline 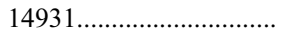 & 1046.3 & 1580.7 & $24.268 \pm 0.005$ & $22.966 \pm 0.004$ \\
\hline 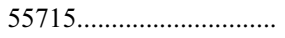 & 1072.6 & 2760.9 & $23.970 \pm 0.006$ & $23.577 \pm 0.008$ \\
\hline 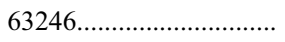 & 1076.5 & 3047.0 & $23.914 \pm 0.006$ & $23.688 \pm 0.014$ \\
\hline $28032 .$. & 1142.6 & 2051.4 & $24.723 \pm 0.016$ & $22.750 \pm 0.020$ \\
\hline $77872 .$. & 1222.6 & 4283.4 & $24.621 \pm 0.007$ & $23.944 \pm 0.009$ \\
\hline $42287 \ldots$ & 1515.6 & 2409.2 & $23.867 \pm 0.004$ & $23.076 \pm 0.009$ \\
\hline $78020 \ldots$ & 1684.4 & 4305.4 & $24.204 \pm 0.008$ & $23.828 \pm 0.014$ \\
\hline $8178 \ldots \ldots$ & 1760.5 & 1138.8 & $23.323 \pm 0.011$ & $21.841 \pm 0.011$ \\
\hline $80670 \ldots \ldots$. & 1832.0 & 5274.4 & $23.401 \pm 0.036$ & $22.541 \pm 0.045$ \\
\hline $76354 \ldots \ldots$ & 1863.1 & 4079.6 & $22.804 \pm 0.006$ & $21.669 \pm 0.008$ \\
\hline $10735 \ldots$ & 2085.9 & 1290.2 & $23.941 \pm 0.008$ & $23.409 \pm 0.006$ \\
\hline $81216 \ldots$ & 2274.6 & 6230.0 & $23.166 \pm 0.008$ & $22.275 \pm 0.010$ \\
\hline $80650 \ldots$ & 2353.3 & 5248.4 & $21.581 \pm 0.006$ & $20.308 \pm 0.010$ \\
\hline $69011 \ldots$ & 2434.6 & 3347.7 & $24.049 \pm 0.006$ & $23.602 \pm 0.015$ \\
\hline $77675 \ldots$ & 2489.6 & 4254.5 & $23.952 \pm 0.005$ & $23.446 \pm 0.005$ \\
\hline 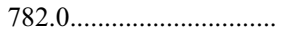 & 2651.1 & 302.98 & $23.553 \pm 0.017$ & $23.119 \pm 0.010$ \\
\hline 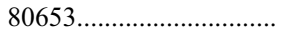 & 2752.0 & 5251.7 & $20.765 \pm 0.005$ & $19.897 \pm 0.006$ \\
\hline 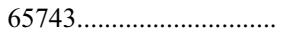 & 2762.8 & 3172.8 & $24.105 \pm 0.011$ & $23.938 \pm 0.013$ \\
\hline 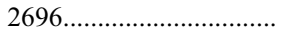 & 2806.1 & 617.02 & $21.481 \pm 0.003$ & $19.757 \pm 0.007$ \\
\hline 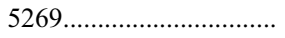 & 2809.2 & 938.09 & $24.405 \pm 0.021$ & $24.123 \pm 0.035$ \\
\hline $64294 \ldots$ & 2867.9 & 3098.7 & $24.445 \pm 0.009$ & $23.901 \pm 0.015$ \\
\hline $3188 \ldots \ldots$. & 2902.8 & 691.54 & $23.705 \pm 0.012$ & $23.084 \pm 0.009$ \\
\hline $12754 \ldots \ldots \ldots$ & 2937.1 & 1439.7 & $22.247 \pm 0.013$ & $19.538 \pm 0.005$ \\
\hline 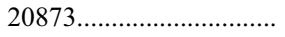 & 3091.0 & 1834.9 & $23.907 \pm 0.008$ & $22.725 \pm 0.019$ \\
\hline 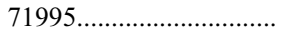 & 3107.3 & 3592.4 & $24.245 \pm 0.005$ & $23.856 \pm 0.010$ \\
\hline $4547 \ldots \ldots \ldots \ldots \ldots \ldots \ldots \ldots$ & 3270.6 & 864.96 & $24.202 \pm 0.005$ & $23.692 \pm 0.008$ \\
\hline 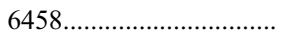 & 3489.1 & 1031.5 & $23.661 \pm 0.006$ & $22.997 \pm 0.009$ \\
\hline 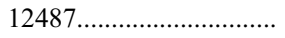 & 3491.3 & 1420.5 & $24.609 \pm 0.016$ & $23.843 \pm 0.025$ \\
\hline 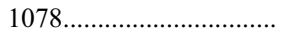 & 3505.2 & 369.76 & $24.453 \pm 0.005$ & $23.957 \pm 0.009$ \\
\hline 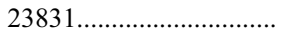 & 3519.9 & 1938.7 & $23.851 \pm 0.007$ & $23.235 \pm 0.024$ \\
\hline $70271 \ldots \ldots$ & 3608.0 & 3438.6 & $24.502 \pm 0.004$ & $23.458 \pm 0.007$ \\
\hline 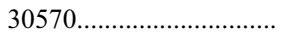 & 3656.4 & 2116.2 & $23.326 \pm 0.004$ & $22.396 \pm 0.005$ \\
\hline 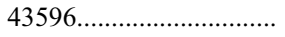 & 3658.0 & 2441.2 & $24.452 \pm 0.007$ & $23.687 \pm 0.014$ \\
\hline 3004 ................................ & 3687.0 & 666.48 & $23.951 \pm 0.008$ & $23.716 \pm 0.013$ \\
\hline 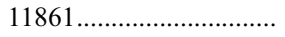 & 3700.1 & 1373.5 & $24.430 \pm 0.005$ & $23.216 \pm 0.016$ \\
\hline 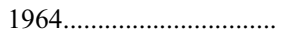 & 3836.9 & 494.27 & $24.306 \pm 0.010$ & $23.669 \pm 0.029$ \\
\hline 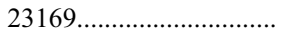 & 3923.7 & 1917.8 & $24.063 \pm 0.010$ & $23.675 \pm 0.015$ \\
\hline $1711 \ldots \ldots \ldots$ & 3937.1 & 456.81 & $24.394 \pm 0.006$ & $23.576 \pm 0.008$ \\
\hline $19359 \ldots \ldots \ldots$ & 4055.0 & 1775.4 & $23.294 \pm 0.008$ & $22.848 \pm 0.010$ \\
\hline $70902 \ldots \ldots$ & 4300.7 & 3498.6 & $23.903 \pm 0.010$ & $22.594 \pm 0.011$ \\
\hline $70124 \ldots \ldots$ & 4376.0 & 3425.1 & $24.155 \pm 0.007$ & $22.553 \pm 0.008$ \\
\hline $4828 \ldots \ldots \ldots \ldots$ & 4539.9 & 896.14 & $24.245 \pm 0.019$ & $23.615 \pm 0.027$ \\
\hline 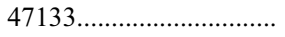 & 4609.8 & 2526.0 & $22.565 \pm 0.008$ & $21.761 \pm 0.007$ \\
\hline 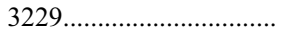 & 5233.7 & 696.14 & $23.404 \pm 0.008$ & $23.346 \pm 0.016$ \\
\hline 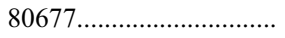 & 5272.6 & 5282.7 & $22.393 \pm 0.004$ & $19.497 \pm 0.009$ \\
\hline 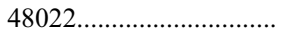 & 5453.4 & 2548.6 & $19.848 \pm 0.009$ & $17.740 \pm 0.039$ \\
\hline 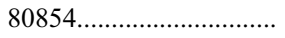 & 5595.6 & 5568.9 & $22.601 \pm 0.009$ & $21.847 \pm 0.011$ \\
\hline 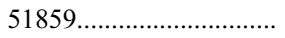 & 6011.7 & 2649.7 & $22.443 \pm 0.005$ & $21.712 \pm 0.009$ \\
\hline
\end{tabular}

${ }^{\text {a }}$ The $x$ and $y$ positions are for pixels of $00^{\prime \prime} 033$ pixel $^{-1}$ as observed in the epoch 1 image with the origin at $x=153, y=137$ in the detector orientation.

b $V$-band and I-band magnitudes have been transformed to the Johnson/Cousins system. Uncertainties are $1 \sigma$.

\subsection{Identification of Cepheid Variables}

We used a number of selection criteria to identify Cepheids. As in Leonard et al. (2003), our criteria were governed by the philosophy that it is better to fail to identify a real Cepheid than to include a non-Cepheid in the sample.

We required that all candidates have reported photometry for all 12 epochs in F555W and all five epochs in F814W, to reduce the risk of aliases in inadequately sampled light curves. We re- quired a modified Welch/Stetson variability index ${ }^{8}$ in excess of 2.50 , a threshold that corresponds to the 99.8 th percentile among the 56,244 measured sources that have all 17 mandatory

${ }^{8}$ This is a ratio of apparent variation over expected precision allowing some degree of correlation among magnitudes measured close together in time (Welch \& Stetson 1993). In the present instance, F555W and F814W magnitudes obtained during the same visits were paired, while the remaining F555W epochs were treated as individual, unmatched observations. 
magnitude measurements. These criteria selected an initial sample of 96 Cepheid candidates, from which we rejected one because it had $\langle V\rangle>28 \mathrm{mag}$, where the photometric errors were judged to be excessively large for our purposes. We verified by inspection that the residuals from the PSF fits of these 95 detections were all consistent with an unresolved source.

Stars meeting these criteria were subjected to a "minimum string length" analysis. This process identified as most likely the trial periods that minimized the sum of magnitude variations for observations at similar phases (Stetson et al. 1998). In this way 20 of the most plausible periods were determined for each candidate. Robust least-squares fits were then performed, comparing the single-epoch magnitudes to template Cepheid light curves, where five parameters representing (1) period, (2) amplitude, (3) epoch of zero phase, (4) mean magnitude in $V$, and (5) mean magnitude in $I$ were free parameters, but the shape of the light curve was a unique function of the assumed period. Templates were fitted in $V$ and $I$ simultaneously, with a single value of the period, phase, and amplitude required for both bandpasses but independent mean magnitudes for the two bands. When the optimum fit had been achieved, the fitted light curves were converted to flux units and numerically integrated over a cycle to achieve flux-weighted mean luminosities in each bandpass; these were then converted back to magnitude units.

Finally, one of us (P. B. S.) applied his experienced judgment to a visual comparison of the data to the adopted template to categorize the individual light-curve fits as "very good," "good," "fair," and "poor." Judgment criteria included the following: (1) When the observed $V$ magnitudes were plotted against a linear increase in epoch, they differed from each other by amounts large compared to their individual measuring uncertainties, and a consistent rise-and-fall pattern of one or more cycles was seen. (2) When phased with the adopted period, the $V$ data were well distributed in phase and showed the classic rapid-rise, slow-decline sawtooth light curve of fundamental-mode radially pulsating variables. (3) All, or nearly all, of the observed $V$ magnitudes agreed with the template light curve by amounts that were consistent with their individual uncertainties. (4) To the extent that the phase sampling allowed us to judge, the $I$-band magnitudes phased to an appropriate light curve with epochs of maximum and minimum brightness coinciding with those in $V$.

This partially subjective judgment was checked by a purely numerical process. Following Leonard et al. (2003), from our empirically derived list of Cepheids we selected those objects whose best-fitting template had a "relative likelihood" greater than 0.3 , where the "relative likelihood" is defined as the quantity $\exp \left(-\chi^{2} / 2 \nu\right)$, with $\nu$ representing the number of degrees of freedom of the fit (in our case, $12 \mathrm{~V}$-band epochs and five $I$-band epochs minus five parameters in the fit yields $\nu=12$, so this limit implies $\chi^{2}<30$ ). Here $\chi^{2}$ is the usual sum over all data points of the square of the model residual normalized by the individual measurement uncertainty. We also implemented a more "robust" version (i.e., more forgiving) of the $\chi^{2}$ threshold by rejecting the single point (among the 17) making the largest contribution to the $\chi^{2}$ sum to reflect the potential for imperfect cosmic-ray rejection for four independent images per epoch.

In all, 35 very good, 29 good, 13 fair, and 18 poor Cepheids were identified by the visual inspection (by P. B. S.). The aforementioned 95 variable candidates are thus reduced to 64 as either very good or good objects selected by inspection. The method of selection by the $\chi^{2}$ criterion was more restrictive, identifying 31 and 51 Cepheids by the $\chi^{2}$ and robust $\chi^{2}$ criteria, respectively. The 70 objects identified as Cepheids by at least one of the methods are presented in Table 4 along with their positions, photometric properties, and the criteria by which they were selected. Their $V$-band and $I$-band light curves are shown in Figure 5 and tabulated in Table 5. For these same objects, their position in the color-magnitude ( $V-I$ vs. $V$ ) diagram of all detected unresolved sources is shown in Figure 6. As expected, the objects identified as Cepheids have $V-I \approx 1.0$ mag, consistent with stars crossing the instability strip.

Obtaining accurate photometry of Cepheids on the crowded and granular background of their hosts is challenging. However, the impact of crowding on the measured magnitudes has been largely eliminated by application of the previously described selection criteria, whose effect has been to disfavor Cepheids that are significantly contaminated (e.g., by a close binary companion). Monte Carlo experiments by the Key Project (Ferrarese et al. 2000) have shown (by reproducing the effects of crowding with artificial PSFs) that the presence of significant contamination will alter the shape parameters of the Cepheid PSF, reducing the amplitude of variation and flattening the sawtooth near minimum light (by contributing a greater fraction to the total flux when the Cepheid is faint). These changes will typically cause a Cepheid candidate to fail one or more of the previous criteria. Ferrarese et al. (2000) found that for multiepoch data, the net crowding bias on the distance modulus is only $\sim 1 \%$. In practice, we found that nearly all of the candidates in locations judged to be "crowded" (defined here as having an additional source within at least 0.1 that contributes at least $\sim 10 \%$ of the peak flux of the variable candidate) failed one or more of the previously discussed selection criteria.

\subsection{The Cepheid Distance to NGC 3370}

In principle, the average luminosity of a Cepheid variable is accurately identified from its period, $P$, via the simple relation

$$
M_{C}=a_{C} \log P+b_{C},
$$

where $M_{C}$ is the intensity-mean absolute magnitude of a Cepheid in a passband $C$, and where $a_{C}$ and $b_{C}$ are the slope and zero point of the relation, respectively. The apparent distance modulus is then given by the apparent and absolute magnitudes,

$$
\mu_{C}=m_{C}-M_{C}
$$

A modest correction to this simple picture is required to allow for the nonzero width of the instability strip: Cepheids of a given luminosity do not necessarily have identically the same radii or, hence, the same effective temperatures. This produces a periodluminosity-color relation among classical Cepheids. The need for a final small correction to account for differences in chemical abundance is addressed in $\S 2.5$.

The use of two or more different passbands allows for the measurement of reddening and the associated correction for extinction to give the true or unreddened distance modulus (also called the "Wesenheit reddening-free modulus"; Madore 1982; Tanvir 1997). For passbands $V$ and $I$ this is given as $\mu_{W}=$ $\mu_{V}-R\left(\mu_{V}-\mu_{I}\right)$, where $R=A_{V} /\left(A_{V}-A_{I}\right)$ is typically taken to be 2.45 as derived from the wavelength-dependent extinction curve of Cardelli et al. (1989). It is fortunate that this is almost exactly the same slope as the relationship between color and magnitude for Cepheids (i.e., for dilute blackbodies) of differing effective temperature at fixed period. Therefore, the reddening correction simultaneously and nearly perfectly removes the 
TABLE 4

Cepheid Candidates

\begin{tabular}{|c|c|c|c|c|c|c|}
\hline ID & $x^{\mathrm{a}}$ & $y^{\mathrm{a}}$ & $\begin{array}{c}P \\
\text { (days) }\end{array}$ & $\langle V\rangle^{\mathrm{b}}$ & $\langle I\rangle^{\mathrm{b}}$ & $\chi^{2} /$ Robust/P. B. S. \\
\hline $71916 \ldots \ldots \ldots \ldots$ & 3109.0 & 3585.7 & 54.0 & $26.54 \pm 0.02$ & $25.47 \pm 0.01$ & NNY \\
\hline $25970 \ldots \ldots \ldots \ldots \ldots$ & 4190.9 & 1996.4 & 36.9 & $26.65 \pm 0.01$ & $25.71 \pm 0.01$ & YYY \\
\hline $8172 \ldots \ldots \ldots \ldots \ldots$ & 3049.7 & 1138.4 & 47.0 & $26.45 \pm 0.01$ & $25.42 \pm 0.01$ & NYY \\
\hline $15128 \ldots \ldots \ldots \ldots \ldots$ & 2431.3 & 1592.5 & 57.3 & $27.05 \pm 0.07$ & $25.71 \pm 0.05$ & NNY \\
\hline 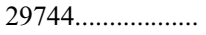 & 4569.8 & 2094.3 & 46.7 & $26.42 \pm 0.01$ & $25.43 \pm 0.01$ & NYY \\
\hline $77676 \ldots \ldots \ldots \ldots$ & 1105.3 & 4254.6 & 22.8 & $27.25 \pm 0.03$ & $26.29 \pm 0.04$ & NNY \\
\hline $33144 \ldots \ldots \ldots \ldots \ldots$ & 3524.9 & 2181.6 & 68.7 & $25.70 \pm 0.01$ & $24.71 \pm 0.01$ & NNY \\
\hline 49796................... & 3540.1 & 2595.1 & 36.1 & $26.55 \pm 0.01$ & $25.65 \pm 0.01$ & $\mathrm{NNY}$ \\
\hline $6853 \ldots \ldots \ldots \ldots \ldots \ldots$ & 3131.0 & 1057.9 & 46.7 & $26.52 \pm 0.02$ & $25.46 \pm 0.01$ & NYY \\
\hline $78473 \ldots \ldots \ldots \ldots \ldots$ & 1507.4 & 4383.9 & 20.5 & $27.18 \pm 0.02$ & $26.32 \pm 0.02$ & YYY \\
\hline $2105 \ldots \ldots \ldots \ldots$ & 3056.1 & 517.14 & 60.0 & $26.05 \pm 0.01$ & $25.17 \pm 0.01$ & YYY \\
\hline 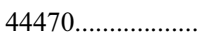 & 1928.2 & 2462.2 & 70.7 & $26.17 \pm 0.01$ & $25.07 \pm 0.02$ & NNY \\
\hline 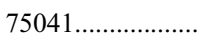 & 1513.9 & 3923.7 & 51.4 & $26.72 \pm 0.01$ & $25.58 \pm 0.01$ & YYY \\
\hline $73063 \ldots \ldots \ldots \ldots$ & 946.42 & 3699.2 & 24.1 & $27.39 \pm 0.02$ & $26.40 \pm 0.03$ & NYY \\
\hline 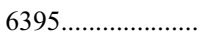 & 3209.7 & 1026.4 & 35.8 & $26.77 \pm 0.02$ & $25.85 \pm 0.04$ & NYY \\
\hline 64774....................... & 3405.8 & 3123.5 & 46.1 & $26.61 \pm 0.02$ & $25.49 \pm 0.02$ & NYY \\
\hline 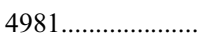 & 4944.8 & 910.62 & 28.6 & $26.75 \pm 0.01$ & $25.92 \pm 0.01$ & YYY \\
\hline 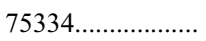 & 595.19 & 3955.8 & 28.9 & $26.89 \pm 0.02$ & $25.95 \pm 0.02$ & NNY \\
\hline $45215 \ldots \ldots \ldots \ldots \ldots \ldots$ & 1546.6 & 2480.2 & 45.0 & $26.48 \pm 0.02$ & $25.52 \pm 0.02$ & NNY \\
\hline $5395 \ldots \ldots \ldots \ldots \ldots$ & 3144.7 & 950.84 & 33.9 & $27.24 \pm 0.02$ & $26.10 \pm 0.02$ & YYY \\
\hline $1171 \ldots \ldots \ldots \ldots \ldots$ & 4381.1 & 386.24 & 24.1 & $27.32 \pm 0.02$ & $26.36 \pm 0.03$ & NNY \\
\hline $56099 \ldots \ldots \ldots \ldots \ldots$ & 2719.7 & 2772.4 & 50.4 & $26.55 \pm 0.02$ & $25.64 \pm 0.02$ & NYY \\
\hline $30818 \ldots \ldots \ldots \ldots \ldots$ & 3617.3 & 2122.6 & 38.9 & $26.16 \pm 0.01$ & $25.46 \pm 0.01$ & NYY \\
\hline 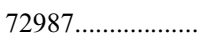 & 1110.1 & 3691.0 & 32.2 & $27.10 \pm 0.02$ & $26.17 \pm 0.02$ & NYY \\
\hline $32112 \ldots \ldots \ldots \ldots \ldots$ & 3289.1 & 2155.4 & 174.0 & $25.84 \pm 0.02$ & $24.62 \pm 0.02$ & NYN \\
\hline $74526 \ldots \ldots \ldots \ldots \ldots$ & 2912.9 & 3867.2 & 27.9 & $27.36 \pm 0.03$ & $26.33 \pm 0.03$ & NNY \\
\hline 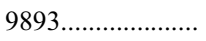 & 2867.7 & 1233.9 & 29.2 & $27.34 \pm 0.04$ & $26.26 \pm 0.05$ & $\mathrm{NNY}$ \\
\hline 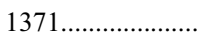 & 4492.1 & 414.96 & 32.7 & $26.54 \pm 0.01$ & $25.37 \pm 0.02$ & NYY \\
\hline $78682 \ldots \ldots \ldots \ldots \ldots$ & 2323.8 & 4428.7 & 37.0 & $27.04 \pm 0.02$ & $26.00 \pm 0.02$ & NNY \\
\hline $32079 \ldots \ldots \ldots \ldots$ & 3275.4 & 2154.6 & 37.6 & $26.39 \pm 0.01$ & $25.50 \pm 0.02$ & YYY \\
\hline $53058 \ldots \ldots \ldots \ldots \ldots$ & 2709.4 & 2681.7 & 31.8 & $26.48 \pm 0.02$ & $25.42 \pm 0.02$ & YYY \\
\hline $5583 \ldots \ldots \ldots \ldots \ldots$ & 3766.4 & 966.75 & 29.3 & $27.12 \pm 0.02$ & $26.23 \pm 0.02$ & NYY \\
\hline $29367 \ldots \ldots \ldots \ldots \ldots$ & 3504.0 & 2085.4 & 42.4 & $26.86 \pm 0.01$ & $25.76 \pm 0.02$ & YYY \\
\hline 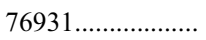 & 1177.6 & 4154.5 & 21.2 & $27.24 \pm 0.02$ & $26.42 \pm 0.02$ & YYY \\
\hline 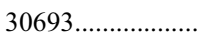 & 3877.3 & 2119.2 & 38.1 & $27.08 \pm 0.02$ & $26.00 \pm 0.03$ & NNY \\
\hline 78887...................... & 1778.0 & 4473.4 & 27.9 & $26.95 \pm 0.02$ & $25.94 \pm 0.02$ & NYY \\
\hline $13149 \ldots \ldots \ldots \ldots \ldots$ & 3775.4 & 1469.1 & 8.2 & $24.89 \pm 0.01$ & $23.87 \pm 0.01$ & YYN \\
\hline $25904 \ldots \ldots \ldots \ldots \ldots$ & 1985.3 & 1994.8 & 28.4 & $26.95 \pm 0.03$ & $26.23 \pm 0.05$ & NYY \\
\hline $24214 \ldots \ldots \ldots \ldots$ & 1909.8 & 1950.4 & 43.1 & $26.98 \pm 0.04$ & $25.88 \pm 0.04$ & NNY \\
\hline $4629 \ldots \ldots \ldots \ldots \ldots \ldots$ & 3473.6 & 874.79 & 23.7 & $27.58 \pm 0.03$ & $26.64 \pm 0.04$ & NYN \\
\hline 59793..................... & 2284.3 & 2894.1 & 35.1 & $27.15 \pm 0.05$ & $26.15 \pm 0.04$ & NNY \\
\hline $2689 \ldots \ldots \ldots \ldots \ldots$ & 4350.8 & 616.04 & 48.8 & $26.59 \pm 0.01$ & $25.59 \pm 0.01$ & YYY \\
\hline $24669 \ldots \ldots \ldots \ldots \ldots$ & 1793.3 & 1962.4 & 31.5 & $26.93 \pm 0.02$ & $25.84 \pm 0.03$ & $\mathrm{NNY}$ \\
\hline $73475 \ldots \ldots \ldots \ldots \ldots$ & 1377.8 & 3753.3 & 24.3 & $27.19 \pm 0.02$ & $25.93 \pm 0.02$ & NYY \\
\hline $5430 \ldots \ldots \ldots \ldots \ldots \ldots$ & 2838.4 & 953.89 & 57.4 & $26.94 \pm 0.01$ & $25.76 \pm 0.02$ & YYN \\
\hline 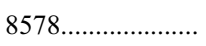 & 3345.0 & 1158.8 & 52.2 & $26.21 \pm 0.01$ & $25.28 \pm 0.01$ & YYY \\
\hline 49963................... & 3064.4 & 2601.2 & 34.9 & $26.90 \pm 0.02$ & $26.19 \pm 0.03$ & NNY \\
\hline 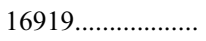 & 3828.3 & 1675.0 & 53.9 & $26.65 \pm 0.02$ & $25.56 \pm 0.02$ & NNY \\
\hline $72780 \ldots \ldots \ldots \ldots \ldots$ & 2368.4 & 3668.1 & 39.7 & $27.14 \pm 0.02$ & $26.11 \pm 0.02$ & YYY \\
\hline $77767 \ldots \ldots \ldots \ldots \ldots$ & 1284.6 & 4267.3 & 30.9 & $27.41 \pm 0.03$ & $26.34 \pm 0.03$ & NNY \\
\hline 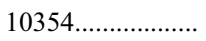 & 3746.7 & 1262.0 & 81.2 & $26.17 \pm 0.01$ & $25.01 \pm 0.01$ & YYN \\
\hline $71460 \ldots \ldots \ldots \ldots \ldots$ & 1636.4 & 3546.8 & 32.5 & $26.73 \pm 0.01$ & $25.92 \pm 0.02$ & YYY \\
\hline $7276 \ldots \ldots \ldots \ldots \ldots \ldots$ & 2846.1 & 1083.7 & 27.6 & $27.22 \pm 0.02$ & $26.20 \pm 0.03$ & YYY \\
\hline $60820 \ldots \ldots \ldots \ldots \ldots$ & 2286.0 & 2939.5 & 46.5 & $26.73 \pm 0.02$ & $25.62 \pm 0.02$ & YYY \\
\hline 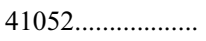 & 1751.1 & 2379.5 & 30.7 & $27.01 \pm 0.02$ & $25.86 \pm 0.02$ & YYY \\
\hline 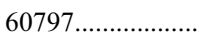 & 1158.7 & 2938.5 & 18.0 & $27.56 \pm 0.02$ & $26.60 \pm 0.03$ & YYY \\
\hline 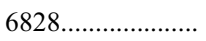 & 2211.1 & 1056.0 & 18.9 & $27.39 \pm 0.02$ & $26.53 \pm 0.03$ & NYN \\
\hline $45125 \ldots \ldots \ldots \ldots \ldots \ldots$ & 3239.8 & 2478.1 & 57.1 & $26.93 \pm 0.02$ & $25.93 \pm 0.03$ & YYN \\
\hline 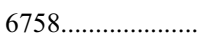 & 3089.4 & 1051.8 & 47.3 & $25.92 \pm 0.01$ & $25.05 \pm 0.02$ & YYY \\
\hline $23676 \ldots \ldots \ldots \ldots \ldots \ldots$ & 3904.3 & 1933.7 & 25.3 & $27.83 \pm 0.05$ & $26.66 \pm 0.03$ & YYY \\
\hline 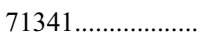 & 3448.2 & 3536.9 & 18.6 & $27.34 \pm 0.02$ & $26.53 \pm 0.02$ & NYY \\
\hline 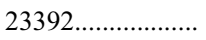 & 2181.7 & 1924.4 & 99.1 & $25.92 \pm 0.01$ & $24.94 \pm 0.01$ & NYN \\
\hline $3412 \ldots \ldots \ldots \ldots \ldots \ldots$ & 3135.4 & 714.73 & 17.6 & $27.28 \pm 0.02$ & $26.58 \pm 0.03$ & YYY \\
\hline $1842 \ldots \ldots \ldots \ldots \ldots \ldots$ & 3234.1 & 474.94 & 34.1 & $27.51 \pm 0.02$ & $26.41 \pm 0.02$ & YYY \\
\hline
\end{tabular}


TABLE 4-Continued

\begin{tabular}{|c|c|c|c|c|c|c|}
\hline ID & $x^{\mathrm{a}}$ & $y^{\mathrm{a}}$ & $\begin{array}{c}P \\
\text { (days) }\end{array}$ & $\langle V\rangle^{\mathrm{b}}$ & $\langle I\rangle^{\mathrm{b}}$ & $\chi^{2} /$ Robust/P. B. S. \\
\hline 79983 ………........... & 1778.0 & 4772.7 & 23.7 & $27.51 \pm 0.03$ & $26.63 \pm 0.03$ & YYY \\
\hline $43163 \ldots \ldots \ldots \ldots \ldots . . .$. & 3711.2 & 2430.3 & 28.7 & $27.22 \pm 0.02$ & $26.24 \pm 0.02$ & YYY \\
\hline 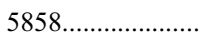 & 3119.6 & 989.13 & 32.3 & $27.12 \pm 0.01$ & $26.11 \pm 0.02$ & YYY \\
\hline $68212 \ldots \ldots \ldots \ldots \ldots . .$. & 2478.7 & 3302.5 & 38.9 & $26.60 \pm 0.02$ & $25.65 \pm 0.03$ & NNY \\
\hline $23445 \ldots \ldots \ldots \ldots \ldots$ & 2342.9 & 1926.2 & 34.4 & $26.94 \pm 0.04$ & $25.92 \pm 0.04$ & NNY \\
\hline $28020 \ldots \ldots \ldots \ldots \ldots \ldots$ & 1938.4 & 2051.1 & 31.7 & $26.51 \pm 0.01$ & $25.80 \pm 0.02$ & YYY \\
\hline $75355 \ldots \ldots \ldots \ldots \ldots$ & 1977.8 & 3959.8 & 35.2 & $27.13 \pm 0.03$ & $25.93 \pm 0.03$ & NYY \\
\hline 19309...................... & 2487.7 & 1773.1 & 33.9 & $26.01 \pm 0.01$ & $25.49 \pm 0.02$ & YYY \\
\hline
\end{tabular}

${ }^{\mathrm{a}}$ The $x$ and $y$ positions are for pixels of $0^{\prime \prime} 033$ pixel $^{-1}$ as observed in the epoch 1 image with the origin at $x=153, y=137$ in the detector orientation.

${ }^{\mathrm{b}} V$-band and $I$-band magnitudes have been transformed to the Johnson/Cousins system. Uncertainties are $1 \sigma$.

complication produced by the nonzero width of the instability strip.

However, the quantitative determination of the best values for the slope and zero point of the resulting period-luminosity (hereafter $P-L$; no color) relation is still subject to ongoing refinement and remains a subject of debate, contributing to past differences between the SKP and the STS groups. The determination of its zero points also suffers from the uncertainty in the distance and reddening to the LMC, which hosts the largest observed sample of Cepheids. Here we have utilized a small set of the most well-founded and likely values of the $P-L$ relations.

Most of the Cepheid analyses by the SKP and the STS groups in the 1990s were referenced to the sample of 32 Cepheids with photoelectric data in the LMC and 1.6 days $<P<63$ days as compiled by Madore \& Freedman (1991, hereafter MF91). However, a significant improvement in the available sample of LMC Cepheids was later made by the Optical Gravitational Lensing Experiment (OGLE; Udalski et al. 1999), which includes $\sim 650$ Cepheids with periods in the range 2.5 days $<P<31$ days and an extremely high rate of data sampling. The photometry from these measurements has been independently verified by Sebo et al. (2002), who also extended the sample to include periods up to 40 days. In their final analysis the SKP adopted the $P-L$ relations derived from the OGLE data. However, a valid criticism of this sample is that $\sim 90 \%$ of the OGLE Cepheids have $P<10$ days, which is significantly shorter than for the faintest Cepheids visible with HST in the hosts of SNe Ia. A more appropriate $P-L$ relation was derived by Thim et al. (2003) and Tammann \& Reindl (2002) by limiting the OGLE Cepheids to the 44 with $P>10$ days. To provide a zero point of these relations for our own analysis, we adopt the canonical distance to the LMC of $\mu=18.50 \pm 0.1 \mathrm{mag}$ as currently used by the SKP group (Freedman et al. 2001, hereafter F01) and the STS group (Saha et al. 2001). Here we consider the $P-L$ relation from only the longer period OGLE data (OGLE+10) chosen by Thim et al. (2003) as our preferred reference sample because of its virtues of utilizing the high-quality, photometrically tested OGLE data while providing a better match to the long-period Cepheids identified with $H S T$. All three aforementioned $P-L$ relations are given in Table 6.

In Figure 7 we show the $V$-band and $I$-band $P$ - $L$ relations of the Cepheids in NGC 3370.

In principle, the mean magnitudes of shorter period (e.g., $P<20$ days), fainter Cepheids (F555W $>27.0 \mathrm{mag}$ ) can be biased by their selection from a magnitude-limited sample. However, the shortest period Cepheid selected by any of our selection methods, $P=17.5$ days, is still detected at $\mathrm{S} / \mathrm{N} \approx 14$ at each F555W epoch. Such a Cepheid whose apparent magnitude was up to 2.0 standard deviations fainter than average by chance (which includes $97.5 \%$ of the sample) could still be detected at $\mathrm{S} / \mathrm{N} \approx 9$ at each epoch. Consequently, no selection bias would be expected for our sample. As an additional test, we measured the distance to NGC 3370 as a function of a minimum period cutoff. As seen in Figure 8, the modulus does not significantly increase for increasing the minimum cutoff as would be expected under the influence of a magnitude limit bias. Therefore, we impose no minimum period cutoff on our sample (higher than the lowest detected period, 17.5 days).

In Table 7 we have utilized the aforementioned Cepheid identification methods, the two independent photometric zero-point determinations, and the three alternative $P-L$ relations to determine the distance to NGC 3370 . The combination that most closely matches the procedures of the SKP is the use of the visual classification, the Stetson zero points, and the $P-L$ relation from F01. This combination results in a distance modulus of $\mu_{w}=32.13 \pm 0.03 \mathrm{mag}$. Alternatively, we can reproduce the distance modulus that would be achieved by the STS collaboration by selecting the Sirianni zero points. The Sirianni et al. (2005) zero points for ACS are a good proxy for the STS zero points because they are similarly derived empirically from the HST throughput, and in addition Sirianni et al. (2005) have shown that their ACS zero points are consistent with the Saha et al. (2001) WFPC2 zero points (for similar fields) to a precision of $0.01 \mathrm{mag}$. To emulate STS, we also use the pre-OGLE $P-L$ relations from MF91. These selections yield a range of $\mu_{w}=32.33-32.38$ mag for the three Cepheid selection criteria; hereafter we assume an average of $\mu_{w}=32.36 \mathrm{mag}$.

However, our goal is not simply to repeat the methodology of these teams but rather to choose what we consider to be the optimal combination of parameters based on the most current available information. It is apparent from the sample size and time sampling of the OGLE Cepheids, as well as the verification by Sebo et al. (2002) of their photometric accuracy, that these data are now preferred in defining the $P-L$ relation. Moreover, in order to compare Cepheids with similar periods free from concerns of a discontinuity in the $P-L$ relation expressed by Tammann \& Reindl (2002), we use the Thim et al. (2003) $P$ - $L$ relation, which utilizes the OGLE data for $P>10$ days. From this we get $\mu_{w}=32.17 \pm$ 0.03 to $32.27 \pm 0.03 \mathrm{mag}$ (where the uncertainties listed are statistical). We use $\mu_{w}=32.22 \pm 0.05$ mag as our best estimate, which is representative of this range but includes a greater uncertainty to account for the systematic uncertainty resulting from the different analyses. 

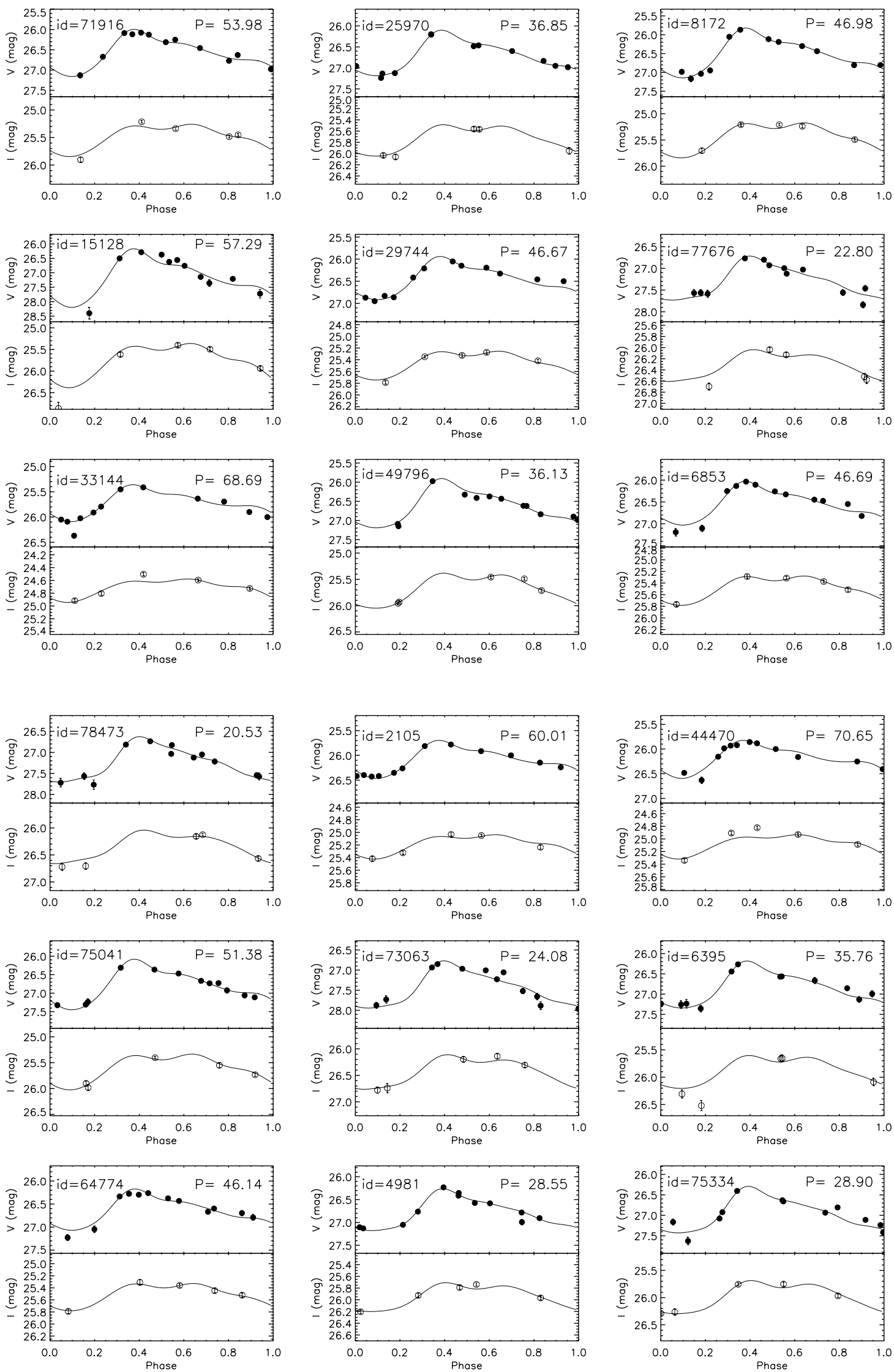

Fig. 5.-F555W and F814W light curves of Cepheids in NGC 3370. For each of the Cepheid candidates listed by identification number in Table 4, the photometry is fitted to the period-specific light-curve template. 

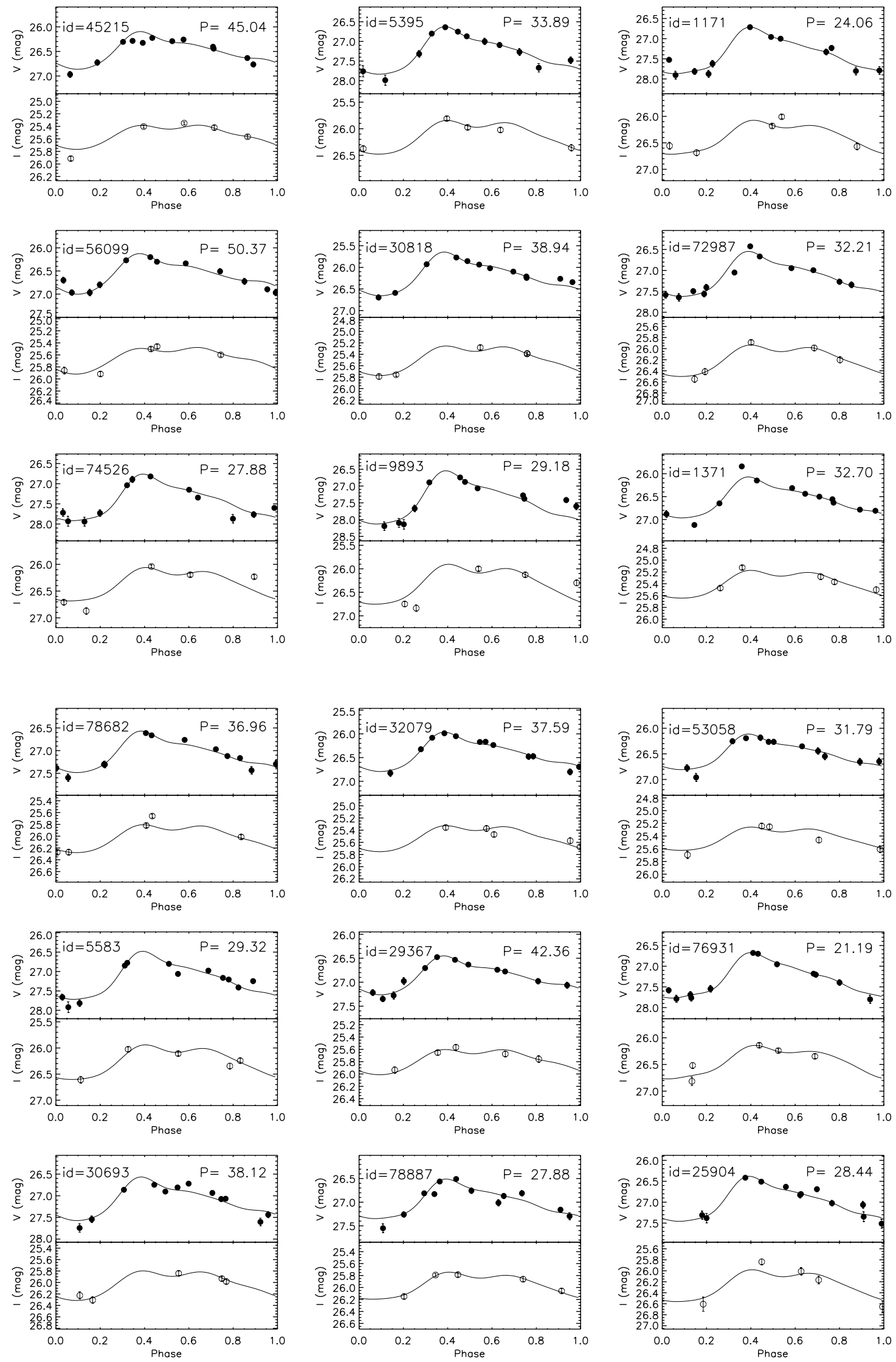

FIg. 5.-Continued 

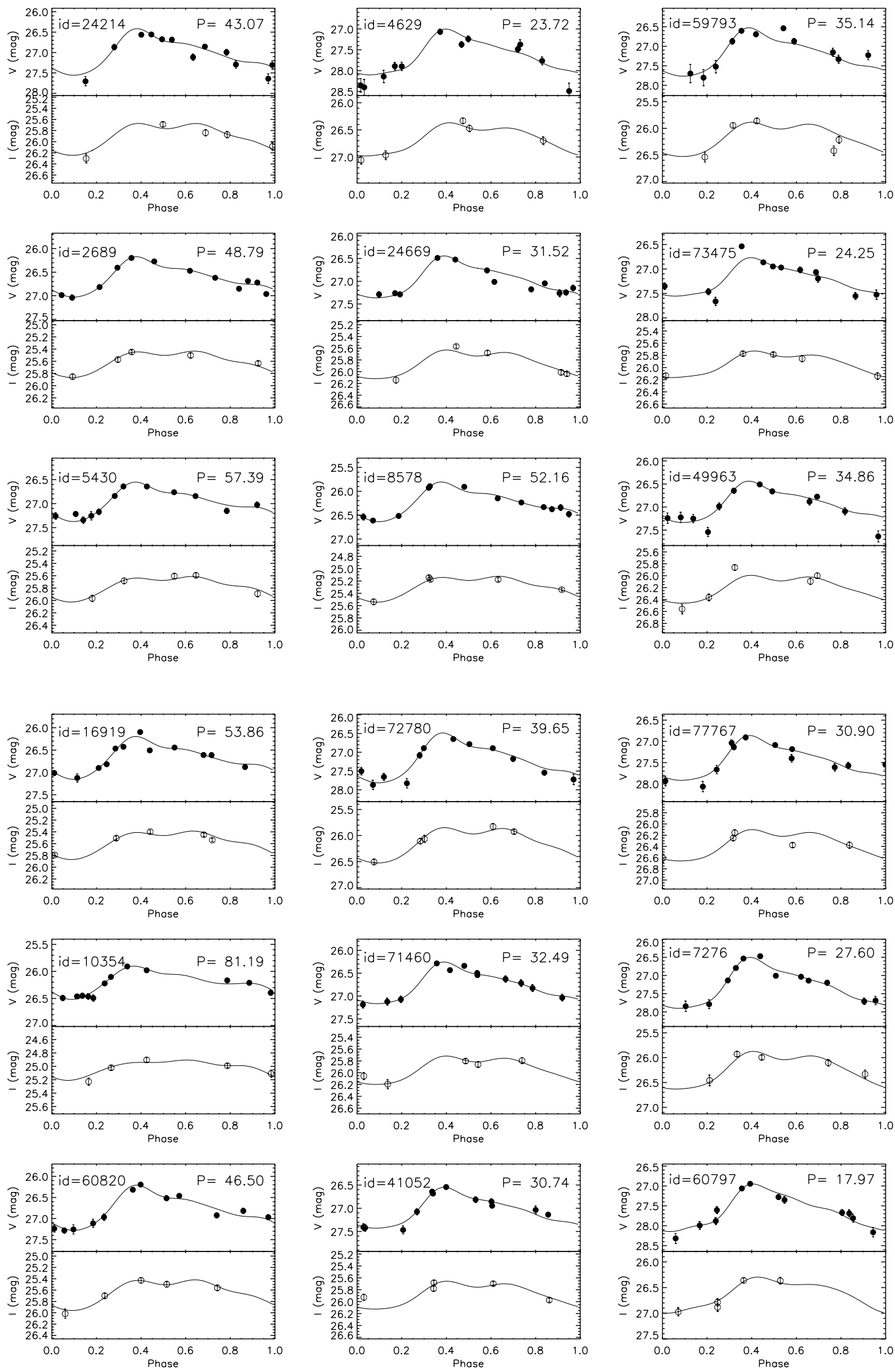

FIG. 5.-Continued 

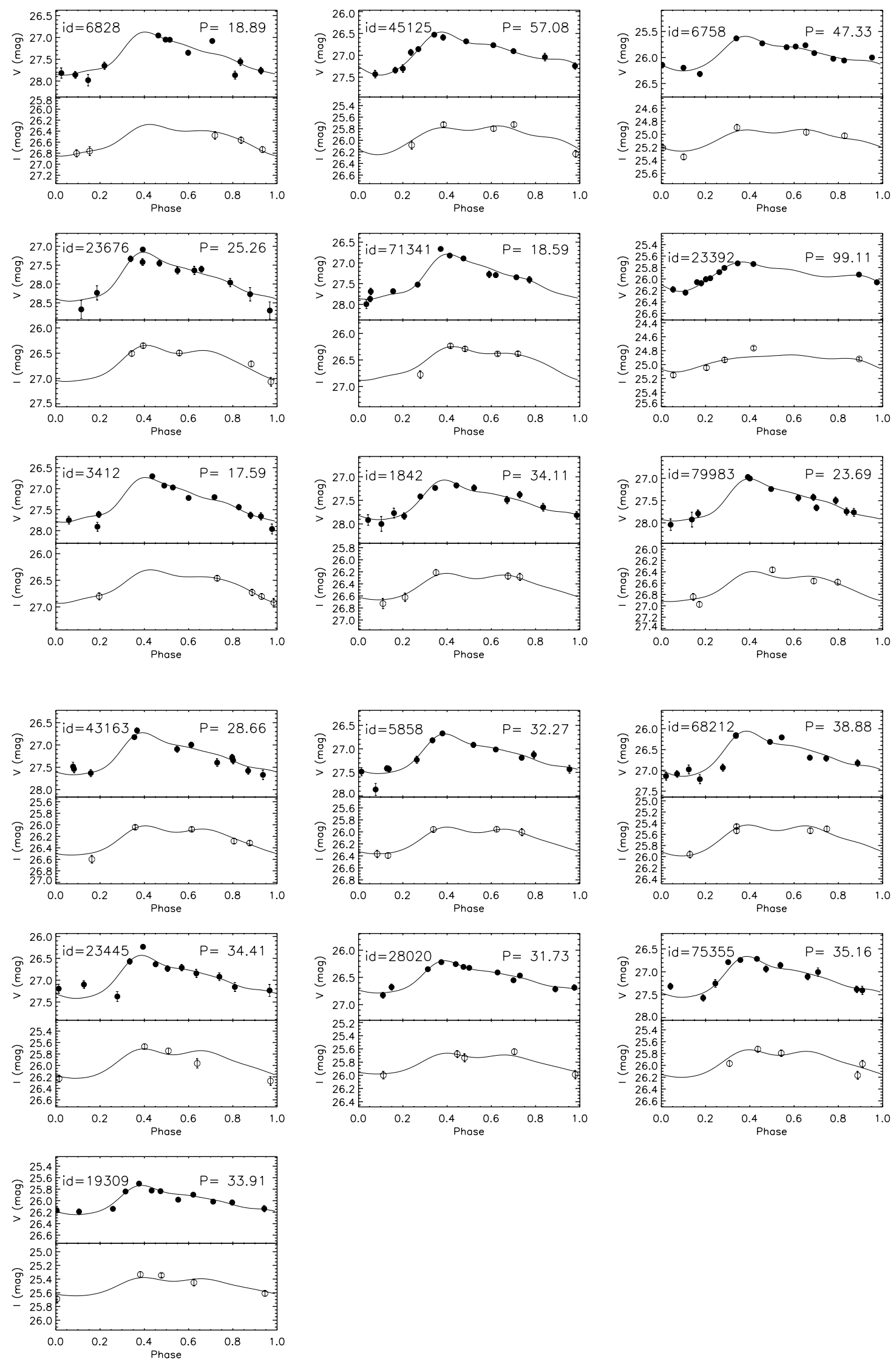

FIG. 5.-Continued 
TABLE 5

PHOTOMETRY OF VARIABLES

\begin{tabular}{|c|c|c|c|c|c|c|}
\hline $\mathrm{MJD}^{\mathrm{a}}$ & 71916 & 25970 & 8172. & 15128 & 29744 & 77676 \\
\hline \multicolumn{7}{|c|}{ F555W } \\
\hline $52729.512 \ldots \ldots \ldots \ldots \ldots \ldots$ & $26.76 \pm 0.05$ & $26.48 \pm 0.03$ & $26.30 \pm 0.03$ & $27.72 \pm 0.14$ & $26.19 \pm 0.03$ & $26.92 \pm 0.04$ \\
\hline $52737.582 \ldots \ldots \ldots \ldots \ldots \ldots$ & $26.62 \pm 0.05$ & $27.13 \pm 0.03$ & $26.19 \pm 0.03$ & $29.12 \pm 0.36$ & $26.14 \pm 0.03$ & $27.58 \pm 0.07$ \\
\hline $52745.387 \ldots \ldots \ldots \ldots \ldots \ldots$ & $26.97 \pm 0.06$ & $26.20 \pm 0.02$ & $26.43 \pm 0.04$ & $28.40 \pm 0.20$ & $26.32 \pm 0.02$ & $27.12 \pm 0.04$ \\
\hline $52750.922 \ldots \ldots \ldots \ldots \ldots \ldots$ & $27.13 \pm 0.06$ & $26.46 \pm 0.03$ & $26.80 \pm 0.06$ & $26.50 \pm 0.03$ & $26.45 \pm 0.03$ & $27.84 \pm 0.07$ \\
\hline $52756.125 \ldots \ldots \ldots \ldots \ldots \ldots$ & $26.67 \pm 0.05$ & $26.59 \pm 0.03$ & $26.80 \pm 0.05$ & $26.28 \pm 0.02$ & $26.49 \pm 0.04$ & $27.56 \pm 0.08$ \\
\hline $52758.055 \ldots \ldots \ldots \ldots \ldots \ldots$ & $26.08 \pm 0.02$ & $26.83 \pm 0.04$ & $26.98 \pm 0.06$ & $26.37 \pm 0.04$ & $26.87 \pm 0.04$ & $26.76 \pm 0.04$ \\
\hline $52760.129 \ldots \ldots \ldots \ldots \ldots \ldots$ & $26.11 \pm 0.03$ & $26.95 \pm 0.04$ & $27.16 \pm 0.08$ & $26.62 \pm 0.03$ & $26.94 \pm 0.03$ & $26.80 \pm 0.04$ \\
\hline $52762.059 \ldots \ldots \ldots \ldots \ldots \ldots$ & $26.07 \pm 0.03$ & $26.98 \pm 0.05$ & $27.03 \pm 0.06$ & $26.55 \pm 0.05$ & $26.83 \pm 0.04$ & $26.99 \pm 0.05$ \\
\hline 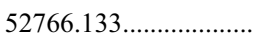 & $26.12 \pm 0.03$ & $26.95 \pm 0.05$ & $26.94 \pm 0.06$ & $26.75 \pm 0.04$ & $26.86 \pm 0.03$ & $27.02 \pm 0.04$ \\
\hline $52768.414 \ldots \ldots \ldots \ldots \ldots \ldots$ & $26.31 \pm 0.04$ & $27.23 \pm 0.05$ & $26.05 \pm 0.03$ & $27.14 \pm 0.07$ & $26.41 \pm 0.02$ & $27.55 \pm 0.06$ \\
\hline $52774.402 \ldots \ldots \ldots \ldots \ldots \ldots \ldots$ & $26.24 \pm 0.04$ & $27.12 \pm 0.04$ & $25.86 \pm 0.03$ & $27.35 \pm 0.11$ & $26.21 \pm 0.03$ & $27.46 \pm 0.06$ \\
\hline $52781.418 \ldots \ldots \ldots \ldots \ldots \ldots \ldots$ & $26.45 \pm 0.03$ & $26.19 \pm 0.03$ & $26.11 \pm 0.03$ & $27.21 \pm 0.07$ & $26.05 \pm 0.02$ & $27.56 \pm 0.07$ \\
\hline \multicolumn{7}{|c|}{ F814W } \\
\hline $52729.645 \ldots \ldots \ldots \ldots \ldots \ldots$ & $25.45 \pm 0.02$ & $26.03 \pm 0.03$ & $25.20 \pm 0.02$ & $26.86 \pm 0.13$ & $25.32 \pm 0.02$ & $26.69 \pm 0.06$ \\
\hline $52745.516 \ldots \ldots \ldots \ldots \ldots \ldots$ & $25.90 \pm 0.04$ & $25.56 \pm 0.03$ & $25.49 \pm 0.03$ & $25.61 \pm 0.04$ & $25.41 \pm 0.02$ & $26.52 \pm 0.05$ \\
\hline $52760.340 \ldots \ldots \ldots \ldots \ldots \ldots$ & $25.21 \pm 0.02$ & $25.95 \pm 0.05$ & $25.70 \pm 0.04$ & $25.40 \pm 0.04$ & $25.78 \pm 0.02$ & $26.12 \pm 0.04$ \\
\hline $52768.547 \ldots \ldots \ldots \ldots \ldots \ldots$ & $25.33 \pm 0.02$ & $26.06 \pm 0.04$ & $25.20 \pm 0.03$ & $25.49 \pm 0.04$ & $25.34 \pm 0.02$ & $26.57 \pm 0.06$ \\
\hline 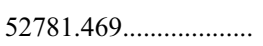 & $25.48 \pm 0.02$ & $25.56 \pm 0.03$ & $25.23 \pm 0.03$ & $25.93 \pm 0.05$ & $25.27 \pm 0.02$ & $26.03 \pm 0.04$ \\
\hline MJD & 33144 & 49796 & 6853. & 78473 & 2105 & 44470 \\
\hline \multicolumn{7}{|c|}{ F555W } \\
\hline $52729.512 \ldots \ldots \ldots \ldots \ldots \ldots$ & $25.41 \pm 0.01$ & $27.09 \pm 0.05$ & $26.54 \pm 0.04$ & $27.05 \pm 0.04$ & $25.78 \pm 0.02$ & $26.16 \pm 0.03$ \\
\hline 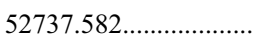 & $25.63 \pm 0.02$ & $26.61 \pm 0.03$ & $26.47 \pm 0.04$ & $27.56 \pm 0.08$ & $25.91 \pm 0.02$ & $26.25 \pm 0.03$ \\
\hline 52745.387..................... & $25.69 \pm 0.02$ & $26.89 \pm 0.04$ & $26.82 \pm 0.03$ & $26.82 \pm 0.03$ & $26.00 \pm 0.03$ & $26.40 \pm 0.03$ \\
\hline $52750.922 \ldots \ldots \ldots \ldots \ldots \ldots$ & $25.90 \pm 0.03$ & $27.15 \pm 0.05$ & $27.19 \pm 0.08$ & $27.54 \pm 0.06$ & $26.14 \pm 0.03$ & $26.48 \pm 0.04$ \\
\hline $52756.125 \ldots \ldots \ldots \ldots \ldots \ldots$ & $26.00 \pm 0.02$ & $25.97 \pm 0.02$ & $27.10 \pm 0.07$ & $27.76 \pm 0.11$ & $26.24 \pm 0.03$ & $26.63 \pm 0.06$ \\
\hline 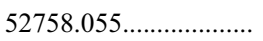 & $26.05 \pm 0.02$ & $26.32 \pm 0.02$ & $26.25 \pm 0.03$ & $26.73 \pm 0.03$ & $26.41 \pm 0.03$ & $26.15 \pm 0.04$ \\
\hline $52760.129 \ldots \ldots \ldots \ldots \ldots \ldots$ & $26.09 \pm 0.02$ & $26.41 \pm 0.03$ & $26.13 \pm 0.02$ & $27.03 \pm 0.05$ & $26.39 \pm 0.03$ & $25.98 \pm 0.03$ \\
\hline $52762.059 \ldots \ldots \ldots \ldots \ldots \ldots$ & $26.37 \pm 0.04$ & $26.37 \pm 0.03$ & $26.03 \pm 0.03$ & $27.12 \pm 0.04$ & $26.42 \pm 0.04$ & $25.93 \pm 0.03$ \\
\hline 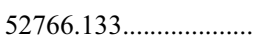 & $26.02 \pm 0.02$ & $26.43 \pm 0.03$ & $26.10 \pm 0.03$ & $27.21 \pm 0.06$ & $26.41 \pm 0.02$ & $25.92 \pm 0.03$ \\
\hline 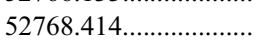 & $25.91 \pm 0.02$ & $26.62 \pm 0.03$ & $26.25 \pm 0.03$ & $27.57 \pm 0.08$ & $26.35 \pm 0.03$ & $25.85 \pm 0.03$ \\
\hline 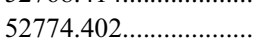 & $25.79 \pm 0.02$ & $26.83 \pm 0.05$ & $26.32 \pm 0.03$ & $27.72 \pm 0.10$ & $26.26 \pm 0.02$ & $25.88 \pm 0.02$ \\
\hline $52781.418 \ldots \ldots \ldots \ldots \ldots \ldots$ & $25.45 \pm 0.02$ & $26.98 \pm 0.04$ & $26.44 \pm 0.04$ & $26.81 \pm 0.04$ & $25.81 \pm 0.02$ & $26.00 \pm 0.03$ \\
\hline \multicolumn{7}{|c|}{ F814W } \\
\hline 52729.645 ...................... & $24.59 \pm 0.01$ & $25.48 \pm 0.02$ & $25.37 \pm 0.02$ & $26.70 \pm 0.06$ & $25.05 \pm 0.02$ & $25.08 \pm 0.02$ \\
\hline 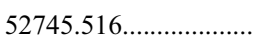 & $24.72 \pm 0.02$ & $25.93 \pm 0.03$ & $25.76 \pm 0.03$ & $26.56 \pm 0.04$ & $25.23 \pm 0.03$ & $25.34 \pm 0.02$ \\
\hline $52760.340 \ldots \ldots \ldots \ldots \ldots \ldots$ & $24.91 \pm 0.02$ & $25.45 \pm 0.02$ & $25.28 \pm 0.02$ & $26.15 \pm 0.04$ & $25.41 \pm 0.03$ & $24.90 \pm 0.02$ \\
\hline $52768.547 \ldots \ldots \ldots \ldots \ldots \ldots$ & $24.80 \pm 0.02$ & $25.71 \pm 0.02$ & $25.31 \pm 0.02$ & $26.72 \pm 0.06$ & $25.32 \pm 0.02$ & $24.82 \pm 0.02$ \\
\hline $52781.469 \ldots \ldots \ldots \ldots \ldots \ldots$ & $24.50 \pm 0.02$ & $25.95 \pm 0.02$ & $25.51 \pm 0.03$ & $26.12 \pm 0.02$ & $25.03 \pm 0.02$ & $24.93 \pm 0.02$ \\
\hline MJD & 75041 & 73063 & 6395. & 64774 & 4981. & 75334 \\
\hline \multicolumn{7}{|c|}{ F555W } \\
\hline $52729.512 \ldots \ldots \ldots \ldots \ldots \ldots$ & $27.23 \pm 0.08$ & $27.22 \pm 0.05$ & $26.56 \pm 0.04$ & $26.69 \pm 0.05$ & $26.76 \pm 0.04$ & $26.80 \pm 0.04$ \\
\hline $52737.582 \ldots \ldots \ldots \ldots \ldots \ldots$ & $27.30 \pm 0.06$ & $26.96 \pm 0.05$ & $27.25 \pm 0.09$ & $26.59 \pm 0.04$ & $26.35 \pm 0.03$ & $27.41 \pm 0.06$ \\
\hline $52745.387 \ldots \ldots \ldots \ldots \ldots \ldots \ldots$ & $26.31 \pm 0.03$ & $27.65 \pm 0.07$ & $26.44 \pm 0.03$ & $26.79 \pm 0.06$ & $26.99 \pm 0.06$ & $26.92 \pm 0.04$ \\
\hline $52750.922 \ldots \ldots \ldots \ldots \ldots \ldots$ & $26.36 \pm 0.04$ & $27.73 \pm 0.09$ & $26.57 \pm 0.05$ & $27.22 \pm 0.06$ & $27.10 \pm 0.05$ & $26.63 \pm 0.03$ \\
\hline $52756.125 \ldots \ldots \ldots \ldots \ldots \ldots$ & $26.46 \pm 0.03$ & $26.85 \pm 0.03$ & $26.66 \pm 0.07$ & $27.04 \pm 0.07$ & $27.05 \pm 0.05$ & $26.93 \pm 0.04$ \\
\hline $52758.055 \ldots \ldots \ldots \ldots \ldots \ldots$ & $26.66 \pm 0.05$ & $27.00 \pm 0.04$ & $26.85 \pm 0.05$ & $26.33 \pm 0.03$ & $26.23 \pm 0.03$ & $27.11 \pm 0.04$ \\
\hline $52760.129 \ldots \ldots \ldots \ldots \ldots \ldots$ & $26.73 \pm 0.05$ & $27.05 \pm 0.04$ & $27.13 \pm 0.07$ & $26.27 \pm 0.03$ & $26.41 \pm 0.03$ & $27.23 \pm 0.05$ \\
\hline $52762.059 \ldots \ldots \ldots \ldots \ldots \ldots \ldots$ & $26.72 \pm 0.04$ & $27.52 \pm 0.06$ & $26.99 \pm 0.07$ & $26.29 \pm 0.03$ & $26.57 \pm 0.04$ & $27.16 \pm 0.07$ \\
\hline $52766.133 \ldots \ldots \ldots \ldots \ldots \ldots$ & $26.92 \pm 0.07$ & $27.88 \pm 0.09$ & $27.23 \pm 0.06$ & $26.26 \pm 0.04$ & $26.58 \pm 0.03$ & $27.62 \pm 0.09$ \\
\hline $52768.414 \ldots \ldots \ldots \ldots \ldots \ldots$ & $27.06 \pm 0.06$ & $27.96 \pm 0.11$ & $27.23 \pm 0.10$ & $26.37 \pm 0.03$ & $26.78 \pm 0.04$ & $27.07 \pm 0.05$ \\
\hline $52774.402 \ldots \ldots \ldots \ldots \ldots \ldots$ & $27.11 \pm 0.05$ & $27.87 \pm 0.07$ & $27.35 \pm 0.08$ & $26.43 \pm 0.03$ & $26.90 \pm 0.04$ & $26.40 \pm 0.03$ \\
\hline $52781.418 \ldots \ldots \ldots \ldots \ldots \ldots$ & $27.32 \pm 0.06$ & $26.93 \pm 0.05$ & $26.26 \pm 0.04$ & $26.66 \pm 0.05$ & $27.13 \pm 0.04$ & $26.65 \pm 0.03$ \\
\hline
\end{tabular}


TABLE 5-Continued

\begin{tabular}{|c|c|c|c|c|c|c|}
\hline MJD & 75041 & 73063 & 6395. & 64774 & 4981. & 75334 \\
\hline \multicolumn{7}{|c|}{ F814W } \\
\hline $52729.645 \ldots \ldots \ldots \ldots \ldots \ldots .$. & $25.90 \pm 0.04$ & $26.19 \pm 0.04$ & $26.30 \pm 0.07$ & $25.44 \pm 0.03$ & $25.78 \pm 0.03$ & $26.29 \pm 0.03$ \\
\hline 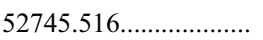 & $25.40 \pm 0.02$ & $26.73 \pm 0.08$ & $25.66 \pm 0.06$ & $25.79 \pm 0.03$ & $26.20 \pm 0.03$ & $25.75 \pm 0.03$ \\
\hline $52760.340 \ldots \ldots \ldots \ldots \ldots \ldots$ & $25.55 \pm 0.04$ & $26.30 \pm 0.04$ & $26.08 \pm 0.06$ & $25.30 \pm 0.04$ & $25.73 \pm 0.02$ & $26.26 \pm 0.06$ \\
\hline 52768.547...................... & $25.73 \pm 0.03$ & $26.77 \pm 0.05$ & $26.52 \pm 0.09$ & $25.36 \pm 0.03$ & $25.96 \pm 0.03$ & $25.75 \pm 0.02$ \\
\hline $52781.469 \ldots \ldots \ldots \ldots \ldots \ldots$ & $25.98 \pm 0.04$ & $26.13 \pm 0.04$ & $25.65 \pm 0.06$ & $25.52 \pm 0.03$ & $25.92 \pm 0.03$ & $25.96 \pm 0.03$ \\
\hline MJD & 45215 & 5395. & 1171. & 56099 & 30818 & 72987 \\
\hline \multicolumn{7}{|c|}{ F555W } \\
\hline $52729.512 \ldots \ldots \ldots \ldots \ldots \ldots$ & $26.63 \pm 0.04$ & $27.76 \pm 0.14$ & $27.52 \pm 0.06$ & $26.30 \pm 0.03$ & $26.69 \pm 0.06$ & $27.27 \pm 0.06$ \\
\hline $52737.582 \ldots \ldots \ldots \ldots \ldots \ldots$ & $26.44 \pm 0.03$ & $26.87 \pm 0.05$ & $27.80 \pm 0.09$ & $26.20 \pm 0.04$ & $26.24 \pm 0.02$ & $27.56 \pm 0.07$ \\
\hline 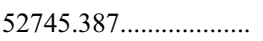 & $26.76 \pm 0.04$ & $27.27 \pm 0.08$ & $27.87 \pm 0.08$ & $26.33 \pm 0.04$ & $26.34 \pm 0.03$ & $26.66 \pm 0.03$ \\
\hline 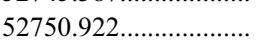 & $26.96 \pm 0.06$ & $27.47 \pm 0.08$ & $27.00 \pm 0.05$ & $26.50 \pm 0.05$ & $26.59 \pm 0.03$ & $26.99 \pm 0.05$ \\
\hline $52756.125 \ldots \ldots \ldots \ldots \ldots \ldots$ & $26.72 \pm 0.05$ & $27.98 \pm 0.12$ & $27.23 \pm 0.06$ & $26.72 \pm 0.06$ & $25.92 \pm 0.02$ & $27.34 \pm 0.06$ \\
\hline $52758.055 \ldots \ldots \ldots \ldots \ldots \ldots$ & $26.30 \pm 0.04$ & $27.31 \pm 0.08$ & $27.78 \pm 0.09$ & $26.89 \pm 0.05$ & $25.77 \pm 0.02$ & $27.58 \pm 0.08$ \\
\hline $52760.129 \ldots \ldots \ldots \ldots \ldots \ldots$ & $26.28 \pm 0.04$ & $26.80 \pm 0.05$ & $27.90 \pm 0.09$ & $26.96 \pm 0.06$ & $25.85 \pm 0.02$ & $27.64 \pm 0.09$ \\
\hline $52762.059 \ldots \ldots \ldots \ldots \ldots \ldots$ & $26.32 \pm 0.03$ & $26.63 \pm 0.05$ & $27.81 \pm 0.07$ & $26.70 \pm 0.06$ & $25.93 \pm 0.03$ & $27.49 \pm 0.06$ \\
\hline 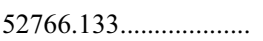 & $26.22 \pm 0.03$ & $26.75 \pm 0.05$ & $27.62 \pm 0.08$ & $26.96 \pm 0.06$ & $26.02 \pm 0.03$ & $27.40 \pm 0.07$ \\
\hline 52768.414..................... & $26.29 \pm 0.03$ & $27.00 \pm 0.08$ & $26.71 \pm 0.04$ & $26.96 \pm 0.07$ & $26.09 \pm 0.02$ & $27.05 \pm 0.05$ \\
\hline $52774.402 \ldots \ldots \ldots \ldots \ldots \ldots$ & $26.25 \pm 0.03$ & $27.09 \pm 0.06$ & $26.95 \pm 0.04$ & $26.79 \pm 0.06$ & $26.21 \pm 0.03$ & $26.42 \pm 0.04$ \\
\hline $52781.418 \ldots \ldots \ldots \ldots \ldots \ldots$ & $26.40 \pm 0.03$ & $27.67 \pm 0.10$ & $27.33 \pm 0.06$ & $26.27 \pm 0.03$ & $26.26 \pm 0.03$ & $26.94 \pm 0.04$ \\
\hline \multicolumn{7}{|c|}{ F814W } \\
\hline $52729.645 \ldots \ldots \ldots \ldots \ldots \ldots$ & $25.42 \pm 0.03$ & $25.97 \pm 0.04$ & $26.56 \pm 0.06$ & $25.50 \pm 0.03$ & $25.38 \pm 0.02$ & $26.41 \pm 0.05$ \\
\hline $52745.516 \ldots \ldots \ldots \ldots \ldots \ldots$ & $25.91 \pm 0.03$ & $26.35 \pm 0.05$ & $26.00 \pm 0.03$ & $25.59 \pm 0.03$ & $25.75 \pm 0.02$ & $25.98 \pm 0.05$ \\
\hline $52760.340 \ldots \ldots \ldots \ldots \ldots \ldots$ & $25.40 \pm 0.02$ & $25.80 \pm 0.03$ & $26.68 \pm 0.05$ & $25.86 \pm 0.05$ & $25.28 \pm 0.04$ & $26.55 \pm 0.05$ \\
\hline 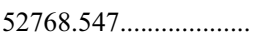 & $25.34 \pm 0.02$ & $26.02 \pm 0.04$ & $26.18 \pm 0.03$ & $25.91 \pm 0.03$ & $25.39 \pm 0.02$ & $25.88 \pm 0.04$ \\
\hline $52781.469 \ldots \ldots \ldots \ldots \ldots$ & $25.56 \pm 0.02$ & $26.37 \pm 0.05$ & $26.55 \pm 0.06$ & $25.46 \pm 0.03$ & $25.78 \pm 0.04$ & $26.20 \pm 0.05$ \\
\hline MJD & 74526 & 9893. & 1371. & 78682 & 32079 & 53058 \\
\hline
\end{tabular}

\begin{tabular}{|c|c|c|c|c|c|c|}
\hline \multicolumn{7}{|c|}{ F555W } \\
\hline $52729.512 .$. & $27.76 \pm 0.07$ & $27.60 \pm 0.09$ & $25.84 \pm 0.02$ & $26.61 \pm 0.03$ & $26.80 \pm 0.06$ & $26.77 \pm 0.06$ \\
\hline $52737.582 \ldots$ & $27.71 \pm 0.09$ & $28.14 \pm 0.14$ & $26.63 \pm 0.03$ & $27.38 \pm 0.06$ & $26.16 \pm 0.04$ & $26.26 \pm 0.04$ \\
\hline 52745.387 ....................... & $27.03 \pm 0.04$ & $26.87 \pm 0.03$ & $26.88 \pm 0.07$ & $27.30 \pm 0.07$ & $26.47 \pm 0.05$ & $26.54 \pm 0.06$ \\
\hline 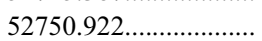 & $27.15 \pm 0.04$ & $27.37 \pm 0.05$ & $26.64 \pm 0.03$ & $26.66 \pm 0.03$ & $26.69 \pm 0.05$ & $26.64 \pm 0.06$ \\
\hline $52756.125 \ldots \ldots \ldots \ldots \ldots \ldots$ & $27.87 \pm 0.10$ & $27.41 \pm 0.07$ & $26.14 \pm 0.02$ & $26.76 \pm 0.03$ & $26.82 \pm 0.06$ & $26.96 \pm 0.07$ \\
\hline $52758.055 \ldots \ldots \ldots \ldots \ldots \ldots \ldots$ & $27.60 \pm 0.05$ & $28.19 \pm 0.12$ & $26.31 \pm 0.03$ & $26.96 \pm 0.04$ & $26.32 \pm 0.05$ & $26.25 \pm 0.04$ \\
\hline $52760.129 \ldots \ldots \ldots \ldots \ldots \ldots \ldots \ldots \ldots$ & $27.93 \pm 0.12$ & $28.09 \pm 0.13$ & $26.43 \pm 0.03$ & $27.12 \pm 0.05$ & $26.08 \pm 0.04$ & $26.19 \pm 0.03$ \\
\hline $52762.059 \ldots \ldots \ldots \ldots \ldots \ldots$ & $27.94 \pm 0.11$ & $27.66 \pm 0.09$ & $26.50 \pm 0.03$ & $27.16 \pm 0.05$ & $25.98 \pm 0.04$ & $26.18 \pm 0.05$ \\
\hline 52766.133....................... & $27.72 \pm 0.08$ & $26.89 \pm 0.05$ & $26.55 \pm 0.04$ & $27.43 \pm 0.08$ & $26.05 \pm 0.03$ & $26.26 \pm 0.05$ \\
\hline 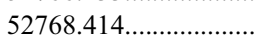 & $26.89 \pm 0.07$ & $26.74 \pm 0.04$ & $26.78 \pm 0.04$ & $27.29 \pm 0.07$ & $26.17 \pm 0.04$ & $26.35 \pm 0.04$ \\
\hline 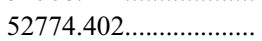 & $26.82 \pm 0.03$ & $27.06 \pm 0.04$ & $26.80 \pm 0.03$ & $27.59 \pm 0.08$ & $26.23 \pm 0.04$ & $26.44 \pm 0.06$ \\
\hline 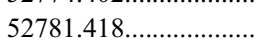 & $27.34 \pm 0.05$ & $27.27 \pm 0.05$ & $27.11 \pm 0.04$ & $27.30 \pm 0.06$ & $26.47 \pm 0.05$ & $26.65 \pm 0.06$ \\
\hline
\end{tabular}

F814W

\begin{tabular}{|c|c|c|c|c|c|c|}
\hline $52729.645 \ldots$ & $26.70 \pm 0.05$ & $26.74 \pm 0.05$ & $25.36 \pm 0.04$ & $26.27 \pm 0.03$ & $25.37 \pm 0.04$ & $25.25 \pm 0.03$ \\
\hline $52745.516 \ldots \ldots \ldots \ldots \ldots \ldots$ & $26.19 \pm 0.04$ & $26.12 \pm 0.04$ & $25.47 \pm 0.04$ & $25.65 \pm 0.03$ & $25.67 \pm 0.04$ & $25.60 \pm 0.05$ \\
\hline 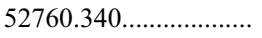 & $26.87 \pm 0.06$ & $26.83 \pm 0.06$ & $25.28 \pm 0.05$ & $26.01 \pm 0.03$ & $25.35 \pm 0.04$ & $25.24 \pm 0.04$ \\
\hline $52768.547 \ldots \ldots \ldots \ldots \ldots \ldots$ & $26.04 \pm 0.03$ & $26.00 \pm 0.04$ & $25.50 \pm 0.04$ & $26.27 \pm 0.04$ & $25.47 \pm 0.03$ & $25.46 \pm 0.04$ \\
\hline $52781.469 \ldots \ldots \ldots \ldots \ldots \ldots$ & $26.23 \pm 0.03$ & $26.29 \pm 0.06$ & $25.12 \pm 0.03$ & $25.81 \pm 0.03$ & $25.57 \pm 0.04$ & $25.69 \pm 0.05$ \\
\hline
\end{tabular}

\begin{tabular}{|c|c|c|c|c|c|c|}
\hline MJD & 5583. & 29367 & 76931 & 30693 & 78887 & 25904 \\
\hline \multicolumn{7}{|c|}{ F555W } \\
\hline $52729.512 \ldots \ldots \ldots \ldots \ldots \ldots$ & $27.06 \pm 0.06$ & $26.77 \pm 0.03$ & $27.76 \pm 0.07$ & $27.74 \pm 0.09$ & $27.26 \pm 0.05$ & $26.51 \pm 0.05$ \\
\hline 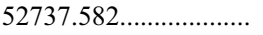 & $27.20 \pm 0.05$ & $26.53 \pm 0.04$ & $27.18 \pm 0.05$ & $27.07 \pm 0.05$ & $26.82 \pm 0.04$ & $26.82 \pm 0.06$ \\
\hline 52745.387........................ & $27.92 \pm 0.13$ & $26.74 \pm 0.04$ & $27.78 \pm 0.08$ & $27.43 \pm 0.06$ & $27.01 \pm 0.06$ & $27.06 \pm 0.08$ \\
\hline $52750.922 \ldots \ldots \ldots \ldots \ldots \ldots$ & $26.77 \pm 0.03$ & $26.97 \pm 0.05$ & $26.69 \pm 0.03$ & $27.54 \pm 0.07$ & $27.15 \pm 0.05$ & $27.30 \pm 0.09$ \\
\hline $52756.125 \ldots \ldots \ldots \ldots \ldots \ldots$ & $26.80 \pm 0.04$ & $27.06 \pm 0.06$ & $27.20 \pm 0.05$ & $26.86 \pm 0.05$ & $27.55 \pm 0.09$ & $26.41 \pm 0.04$ \\
\hline $52758.055 \ldots \ldots \ldots \ldots \ldots \ldots$ & $26.97 \pm 0.04$ & $27.21 \pm 0.06$ & $27.80 \pm 0.09$ & $26.74 \pm 0.03$ & $26.81 \pm 0.04$ & $26.63 \pm 0.05$ \\
\hline $52760.129 \ldots \ldots \ldots \ldots \ldots \ldots$ & $27.16 \pm 0.05$ & $27.34 \pm 0.05$ & $27.58 \pm 0.06$ & $26.90 \pm 0.05$ & $26.56 \pm 0.04$ & $26.80 \pm 0.05$ \\
\hline $52762.059 \ldots \ldots \ldots \ldots \ldots \ldots$ & $27.41 \pm 0.06$ & $27.27 \pm 0.07$ & $27.68 \pm 0.07$ & $26.80 \pm 0.03$ & $26.50 \pm 0.04$ & $26.68 \pm 0.05$ \\
\hline 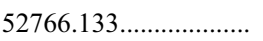 & $27.24 \pm 0.06$ & $26.97 \pm 0.07$ & $27.54 \pm 0.08$ & $26.72 \pm 0.04$ & $26.75 \pm 0.05$ & $27.02 \pm 0.06$ \\
\hline 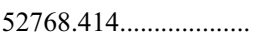 & $27.66 \pm 0.07$ & $26.70 \pm 0.04$ & $26.67 \pm 0.03$ & $26.93 \pm 0.05$ & $26.86 \pm 0.05$ & $27.34 \pm 0.11$ \\
\hline $52774.402 \ldots \ldots \ldots \ldots \ldots \ldots$ & $27.81 \pm 0.08$ & $26.47 \pm 0.04$ & $26.95 \pm 0.05$ & $27.06 \pm 0.04$ & $26.81 \pm 0.05$ & $27.51 \pm 0.10$ \\
\hline $52781.418 \ldots \ldots \ldots \ldots \ldots \ldots$ & $26.84 \pm 0.03$ & $26.63 \pm 0.03$ & $27.39 \pm 0.06$ & $27.60 \pm 0.08$ & $27.29 \pm 0.07$ & $27.38 \pm 0.11$ \\
\hline
\end{tabular}


TABLE 5-Continued

\begin{tabular}{|c|c|c|c|c|c|c|}
\hline MJD & 5583. & 29367 & 76931 & 30693 & 78887 & 25904 \\
\hline \multicolumn{7}{|c|}{ F814W } \\
\hline $52729.645 \ldots \ldots \ldots \ldots \ldots \ldots$ & $26.34 \pm 0.05$ & $25.56 \pm 0.04$ & $26.34 \pm 0.04$ & $25.93 \pm 0.04$ & $25.79 \pm 0.02$ & $26.01 \pm 0.06$ \\
\hline $52745.516 \ldots \ldots \ldots \ldots \ldots \ldots \ldots$ & $26.02 \pm 0.04$ & $25.75 \pm 0.05$ & $26.14 \pm 0.04$ & $26.30 \pm 0.05$ & $26.05 \pm 0.04$ & $26.60 \pm 0.12$ \\
\hline $52760.340 \ldots \ldots \ldots \ldots \ldots \ldots$ & $26.24 \pm 0.04$ & $25.93 \pm 0.05$ & $26.51 \pm 0.05$ & $25.84 \pm 0.04$ & $25.78 \pm 0.04$ & $26.16 \pm 0.07$ \\
\hline $52768.547 \ldots \ldots \ldots \ldots \ldots \ldots$ & $26.60 \pm 0.06$ & $25.65 \pm 0.04$ & $26.24 \pm 0.03$ & $25.98 \pm 0.04$ & $25.86 \pm 0.04$ & $26.65 \pm 0.10$ \\
\hline $52781.469 \ldots \ldots \ldots \ldots \ldots \ldots \ldots$ & $26.10 \pm 0.04$ & $25.67 \pm 0.04$ & $26.81 \pm 0.08$ & $26.22 \pm 0.06$ & $26.15 \pm 0.04$ & $25.83 \pm 0.05$ \\
\hline MJD & 24214 & 4629. & 59793 & 2689. & 24669 & 73475 \\
\hline \multicolumn{7}{|c|}{ F555W } \\
\hline $52729.512 \ldots \ldots \ldots \ldots \ldots \ldots$ & $27.30 \pm 0.10$ & $28.35 \pm 0.16$ & $27.33 \pm 0.10$ & $26.19 \pm 0.04$ & $26.76 \pm 0.05$ & $26.94 \pm 0.04$ \\
\hline $52737.582 \ldots \ldots \ldots \ldots \ldots \ldots$ & $26.99 \pm 0.07$ & $27.76 \pm 0.09$ & $26.87 \pm 0.07$ & $26.40 \pm 0.04$ & $27.24 \pm 0.06$ & $26.53 \pm 0.03$ \\
\hline $52745.387 \ldots \ldots \ldots \ldots \ldots \ldots$ & $27.64 \pm 0.12$ & $27.89 \pm 0.09$ & $26.53 \pm 0.06$ & $26.27 \pm 0.04$ & $27.29 \pm 0.05$ & $27.06 \pm 0.05$ \\
\hline $52750.922 \ldots \ldots \ldots \ldots \ldots \ldots$ & $27.70 \pm 0.11$ & $27.23 \pm 0.07$ & $27.15 \pm 0.10$ & $26.47 \pm 0.03$ & $26.52 \pm 0.03$ & $27.35 \pm 0.06$ \\
\hline $52756.125 \ldots$ & $26.86 \pm 0.07$ & $27.37 \pm 0.11$ & $27.22 \pm 0.11$ & $26.62 \pm 0.03$ & $27.01 \pm 0.05$ & $27.66 \pm 0.08$ \\
\hline $52758.055 \ldots \ldots \ldots \ldots \ldots \ldots$ & $26.56 \pm 0.04$ & $28.49 \pm 0.18$ & $28.51 \pm 0.33$ & $26.85 \pm 0.05$ & $27.17 \pm 0.05$ & $26.86 \pm 0.04$ \\
\hline $52760.129 \ldots \ldots \ldots \ldots \ldots \ldots$ & $26.55 \pm 0.05$ & $28.40 \pm 0.18$ & $27.69 \pm 0.23$ & $26.68 \pm 0.04$ & $27.04 \pm 0.04$ & $26.97 \pm 0.04$ \\
\hline $52762.059 \ldots \ldots \ldots \ldots \ldots \ldots$ & $26.67 \pm 0.05$ & $28.14 \pm 0.14$ & $27.80 \pm 0.21$ & $26.72 \pm 0.05$ & $27.26 \pm 0.07$ & $27.01 \pm 0.06$ \\
\hline $52766.133 \ldots \ldots \ldots \ldots \ldots \ldots$ & $26.68 \pm 0.06$ & $27.89 \pm 0.10$ & $27.52 \pm 0.16$ & $26.96 \pm 0.04$ & $27.14 \pm 0.06$ & $27.19 \pm 0.07$ \\
\hline 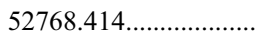 & $27.11 \pm 0.07$ & $27.06 \pm 0.06$ & $26.60 \pm 0.05$ & $26.98 \pm 0.04$ & $27.29 \pm 0.06$ & $27.55 \pm 0.07$ \\
\hline 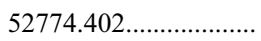 & $26.85 \pm 0.05$ & $27.37 \pm 0.07$ & $26.69 \pm 0.07$ & $27.04 \pm 0.05$ & $27.26 \pm 0.05$ & $27.52 \pm 0.09$ \\
\hline 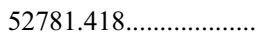 & $27.29 \pm 0.09$ & $27.48 \pm 0.07$ & $26.87 \pm 0.07$ & $26.81 \pm 0.04$ & $26.48 \pm 0.03$ & $27.46 \pm 0.06$ \\
\hline \multicolumn{7}{|c|}{ F814W } \\
\hline $52729.645 \ldots \ldots \ldots \ldots \ldots \ldots$ & $25.87 \pm 0.05$ & $26.69 \pm 0.07$ & $25.94 \pm 0.05$ & $25.57 \pm 0.04$ & $26.03 \pm 0.03$ & $25.77 \pm 0.03$ \\
\hline $52745.516 \ldots \ldots \ldots \ldots \ldots \ldots$ & $26.30 \pm 0.08$ & $26.47 \pm 0.06$ & $26.42 \pm 0.09$ & $25.50 \pm 0.03$ & $25.56 \pm 0.02$ & $26.13 \pm 0.04$ \\
\hline $52760.340 \ldots \ldots \ldots \ldots \ldots \ldots$ & $25.68 \pm 0.04$ & $26.96 \pm 0.08$ & $26.54 \pm 0.09$ & $25.63 \pm 0.03$ & $26.01 \pm 0.04$ & $25.85 \pm 0.05$ \\
\hline $52768.547 \ldots \ldots \ldots \ldots \ldots \ldots$ & $25.83 \pm 0.05$ & $26.33 \pm 0.05$ & $25.86 \pm 0.05$ & $25.85 \pm 0.03$ & $26.14 \pm 0.04$ & $26.14 \pm 0.05$ \\
\hline 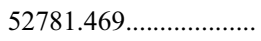 & $26.08 \pm 0.07$ & $27.05 \pm 0.07$ & $26.21 \pm 0.06$ & $25.44 \pm 0.03$ & $25.68 \pm 0.04$ & $25.78 \pm 0.03$ \\
\hline MJD & 5430. & 8578. & 49963 & 16919 & 72780 & 77767 \\
\hline \multicolumn{7}{|c|}{ F555W } \\
\hline $52729.512 \ldots \ldots \ldots \ldots \ldots \ldots$ & $26.76 \pm 0.03$ & $25.92 \pm 0.03$ & $26.77 \pm 0.05$ & $26.61 \pm 0.05$ & $26.89 \pm 0.05$ & $27.54 \pm 0.11$ \\
\hline 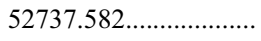 & $26.84 \pm 0.04$ & $25.89 \pm 0.03$ & $27.54 \pm 0.09$ & $26.61 \pm 0.05$ & $26.89 \pm 0.04$ & $27.13 \pm 0.06$ \\
\hline 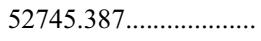 & $27.15 \pm 0.05$ & $25.90 \pm 0.03$ & $26.50 \pm 0.04$ & $26.88 \pm 0.05$ & $26.78 \pm 0.04$ & $27.18 \pm 0.05$ \\
\hline $52750.922 \ldots \ldots \ldots \ldots \ldots \ldots$ & $27.02 \pm 0.06$ & $26.14 \pm 0.04$ & $26.88 \pm 0.07$ & $27.01 \pm 0.05$ & $27.17 \pm 0.07$ & $27.57 \pm 0.07$ \\
\hline $52756.125 \ldots \ldots \ldots \ldots \ldots \ldots \ldots$ & $27.25 \pm 0.07$ & $26.23 \pm 0.03$ & $27.09 \pm 0.08$ & $27.12 \pm 0.09$ & $27.54 \pm 0.06$ & $27.93 \pm 0.11$ \\
\hline $52758.055 \ldots \ldots \ldots \ldots \ldots \ldots$ & $27.21 \pm 0.05$ & $26.33 \pm 0.04$ & $27.63 \pm 0.12$ & $26.90 \pm 0.04$ & $27.72 \pm 0.13$ & $28.06 \pm 0.12$ \\
\hline $52760.129 \ldots \ldots \ldots \ldots \ldots \ldots$ & $27.34 \pm 0.06$ & $26.37 \pm 0.05$ & $27.24 \pm 0.11$ & $26.81 \pm 0.05$ & $27.50 \pm 0.09$ & $27.66 \pm 0.10$ \\
\hline $52762.059 \ldots \ldots \ldots \ldots \ldots \ldots$ & $27.25 \pm 0.08$ & $26.34 \pm 0.06$ & $27.22 \pm 0.11$ & $26.46 \pm 0.03$ & $27.87 \pm 0.12$ & $27.03 \pm 0.07$ \\
\hline $52766.133 \ldots \ldots \ldots \ldots \ldots \ldots$ & $27.17 \pm 0.05$ & $26.48 \pm 0.06$ & $27.25 \pm 0.09$ & $26.42 \pm 0.04$ & $27.65 \pm 0.09$ & $26.90 \pm 0.05$ \\
\hline 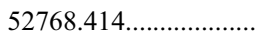 & $26.84 \pm 0.04$ & $26.53 \pm 0.06$ & $26.98 \pm 0.07$ & $26.09 \pm 0.02$ & $27.82 \pm 0.12$ & $27.08 \pm 0.05$ \\
\hline $52774.402 \ldots \ldots \ldots \ldots \ldots \ldots$ & $26.64 \pm 0.04$ & $26.61 \pm 0.04$ & $26.64 \pm 0.05$ & $26.50 \pm 0.04$ & $27.08 \pm 0.07$ & $27.40 \pm 0.09$ \\
\hline 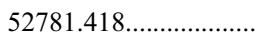 & $26.64 \pm 0.04$ & $26.51 \pm 0.04$ & $26.66 \pm 0.05$ & $26.44 \pm 0.04$ & $26.64 \pm 0.05$ & $27.60 \pm 0.08$ \\
\hline \multicolumn{7}{|c|}{ F814W } \\
\hline $52729.645 \ldots \ldots \ldots \ldots \ldots \ldots$ & $25.59 \pm 0.04$ & $25.17 \pm 0.02$ & $26.36 \pm 0.05$ & $25.53 \pm 0.02$ & $26.06 \pm 0.06$ & $26.15 \pm 0.05$ \\
\hline 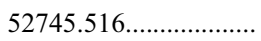 & $25.89 \pm 0.04$ & $25.17 \pm 0.02$ & $26.09 \pm 0.05$ & $25.79 \pm 0.02$ & $25.92 \pm 0.04$ & $26.37 \pm 0.05$ \\
\hline $52760.340 \ldots \ldots \ldots \ldots \ldots \ldots$ & $25.96 \pm 0.05$ & $25.34 \pm 0.02$ & $26.55 \pm 0.08$ & $25.50 \pm 0.03$ & $26.50 \pm 0.05$ & $26.25 \pm 0.04$ \\
\hline $52768.547 \ldots \ldots \ldots \ldots \ldots \ldots \ldots$ & $25.68 \pm 0.04$ & $25.53 \pm 0.04$ & $25.86 \pm 0.04$ & $25.39 \pm 0.02$ & $26.10 \pm 0.04$ & $26.37 \pm 0.04$ \\
\hline $52781.469 \ldots \ldots \ldots \ldots \ldots \ldots$ & $25.60 \pm 0.04$ & $25.14 \pm 0.03$ & $26.00 \pm 0.04$ & $25.44 \pm 0.03$ & $25.82 \pm 0.06$ & $26.60 \pm 0.07$ \\
\hline MJD & 10354 & 71460 & 7276. & 60820 & 41052 & 60797 \\
\hline \multicolumn{7}{|c|}{ F555W } \\
\hline $52729.512 \ldots \ldots \ldots \ldots \ldots \ldots$ & $25.97 \pm 0.03$ & $27.12 \pm 0.07$ & $27.78 \pm 0.12$ & $26.51 \pm 0.06$ & $27.40 \pm 0.07$ & $27.60 \pm 0.08$ \\
\hline $52737.582 \ldots \ldots \ldots \ldots \ldots \ldots$ & $26.16 \pm 0.04$ & $26.49 \pm 0.05$ & $26.79 \pm 0.04$ & $26.19 \pm 0.04$ & $26.68 \pm 0.04$ & $27.06 \pm 0.05$ \\
\hline $52745.387 \ldots \ldots \ldots \ldots \ldots \ldots$ & $26.21 \pm 0.04$ & $26.82 \pm 0.07$ & $27.03 \pm 0.06$ & $26.46 \pm 0.05$ & $26.85 \pm 0.06$ & $27.67 \pm 0.07$ \\
\hline $52750.922 \ldots \ldots \ldots \ldots \ldots \ldots$ & $26.39 \pm 0.04$ & $27.18 \pm 0.07$ & $27.71 \pm 0.08$ & $26.92 \pm 0.06$ & $27.13 \pm 0.05$ & $27.88 \pm 0.08$ \\
\hline $52756.125 \ldots \ldots \ldots \ldots \ldots \ldots$ & $26.49 \pm 0.05$ & $27.07 \pm 0.07$ & $27.84 \pm 0.14$ & $26.81 \pm 0.06$ & $27.41 \pm 0.07$ & $27.35 \pm 0.08$ \\
\hline $52758.055 \ldots \ldots \ldots \ldots \ldots \ldots$ & $26.46 \pm 0.04$ & $26.28 \pm 0.04$ & $27.13 \pm 0.05$ & $26.96 \pm 0.05$ & $27.46 \pm 0.08$ & $27.68 \pm 0.09$ \\
\hline $52760.129 \ldots \ldots \ldots \ldots \ldots \ldots$ & $26.45 \pm 0.04$ & $26.43 \pm 0.05$ & $26.53 \pm 0.04$ & $27.23 \pm 0.08$ & $27.07 \pm 0.06$ & $28.16 \pm 0.11$ \\
\hline $52762.059 \ldots \ldots \ldots \ldots \ldots \ldots$ & $26.46 \pm 0.05$ & $26.33 \pm 0.05$ & $26.47 \pm 0.05$ & $27.28 \pm 0.06$ & $26.64 \pm 0.04$ & $28.31 \pm 0.12$ \\
\hline $52766.133 \ldots \ldots \ldots \ldots \ldots \ldots$ & $26.49 \pm 0.05$ & $26.54 \pm 0.04$ & $27.00 \pm 0.05$ & $27.25 \pm 0.11$ & $26.54 \pm 0.04$ & $27.99 \pm 0.09$ \\
\hline 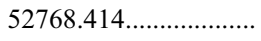 & $26.22 \pm 0.04$ & $26.62 \pm 0.06$ & $27.14 \pm 0.06$ & $27.11 \pm 0.09$ & $26.81 \pm 0.06$ & $26.94 \pm 0.04$ \\
\hline $52774.402 \ldots \ldots \ldots \ldots \ldots \ldots$ & $26.10 \pm 0.04$ & $26.71 \pm 0.07$ & $27.19 \pm 0.07$ & $26.96 \pm 0.07$ & $26.94 \pm 0.05$ & $27.27 \pm 0.06$ \\
\hline $52781.418 \ldots \ldots \ldots \ldots \ldots \ldots . . . . . . .$. & $25.90 \pm 0.04$ & $27.03 \pm 0.07$ & $27.68 \pm 0.11$ & $26.31 \pm 0.04$ & $27.03 \pm 0.08$ & $27.80 \pm 0.10$ \\
\hline
\end{tabular}


TABLE 5-Continued

\begin{tabular}{|c|c|c|c|c|c|c|}
\hline MJD & 10354 & 71460 & 7276. & 60820 & 41052 & 60797 \\
\hline \multicolumn{7}{|c|}{ F814W } \\
\hline $52729.645 \ldots \ldots \ldots \ldots \ldots \ldots$ & $24.99 \pm 0.03$ & $25.86 \pm 0.04$ & $25.92 \pm 0.05$ & $25.42 \pm 0.04$ & $25.68 \pm 0.03$ & $26.35 \pm 0.05$ \\
\hline $52745.516 \ldots \ldots \ldots \ldots \ldots \ldots$ & $25.10 \pm 0.05$ & $26.05 \pm 0.06$ & $26.33 \pm 0.08$ & $25.56 \pm 0.04$ & $25.97 \pm 0.03$ & $26.78 \pm 0.07$ \\
\hline $52760.340 \ldots \ldots \ldots \ldots \ldots \ldots$ & $25.22 \pm 0.05$ & $25.80 \pm 0.03$ & $25.99 \pm 0.04$ & $26.01 \pm 0.08$ & $25.78 \pm 0.04$ & $26.97 \pm 0.08$ \\
\hline 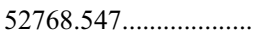 & $25.02 \pm 0.03$ & $25.79 \pm 0.05$ & $26.10 \pm 0.06$ & $25.70 \pm 0.04$ & $25.69 \pm 0.03$ & $26.36 \pm 0.06$ \\
\hline $52781.469 \ldots \ldots \ldots \ldots \ldots \ldots \ldots$ & $24.90 \pm 0.03$ & $26.19 \pm 0.07$ & $26.45 \pm 0.10$ & $25.49 \pm 0.05$ & $25.92 \pm 0.04$ & $26.88 \pm 0.08$ \\
\hline MJD & 6828. & 45125 & 6758. & 23676 & 71341 & 23392 \\
\hline \multicolumn{7}{|c|}{ F555W } \\
\hline $52729.512 \ldots \ldots \ldots \ldots \ldots \ldots$ & $27.55 \pm 0.08$ & $26.76 \pm 0.04$ & $26.19 \pm 0.04$ & $27.08 \pm 0.05$ & $26.82 \pm 0.04$ & $25.73 \pm 0.03$ \\
\hline $52737.582 \ldots \ldots \ldots \ldots \ldots \ldots . . . . . . .$. & $27.85 \pm 0.07$ & $26.90 \pm 0.05$ & $26.14 \pm 0.05$ & $27.33 \pm 0.07$ & $27.29 \pm 0.06$ & $25.92 \pm 0.04$ \\
\hline 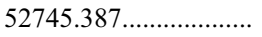 & $27.05 \pm 0.03$ & $27.04 \pm 0.08$ & $26.31 \pm 0.04$ & $27.60 \pm 0.08$ & $27.68 \pm 0.07$ & $26.05 \pm 0.04$ \\
\hline $52750.922 \ldots \ldots \ldots \ldots \ldots \ldots$ & $27.76 \pm 0.07$ & $27.24 \pm 0.07$ & $25.63 \pm 0.04$ & $28.70 \pm 0.22$ & $26.89 \pm 0.03$ & $26.18 \pm 0.04$ \\
\hline $52756.125 \ldots$ & $27.64 \pm 0.08$ & $27.43 \pm 0.08$ & $25.72 \pm 0.03$ & $28.23 \pm 0.18$ & $27.40 \pm 0.07$ & $26.23 \pm 0.04$ \\
\hline $52758.055 \ldots \ldots \ldots \ldots \ldots \ldots$ & $27.05 \pm 0.04$ & $27.34 \pm 0.06$ & $25.80 \pm 0.04$ & $27.42 \pm 0.09$ & $27.86 \pm 0.07$ & $26.05 \pm 0.03$ \\
\hline $52760.129 \ldots \ldots \ldots \ldots \ldots \ldots \ldots$ & $27.35 \pm 0.05$ & $27.30 \pm 0.08$ & $25.79 \pm 0.04$ & $27.45 \pm 0.07$ & $27.68 \pm 0.06$ & $26.07 \pm 0.04$ \\
\hline 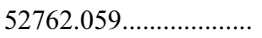 & $27.08 \pm 0.05$ & $26.93 \pm 0.06$ & $25.76 \pm 0.04$ & $27.64 \pm 0.09$ & $27.52 \pm 0.05$ & $26.00 \pm 0.04$ \\
\hline $52766.133 \ldots \ldots \ldots \ldots \ldots \ldots . . . . . .$. & $27.86 \pm 0.09$ & $26.85 \pm 0.05$ & $25.91 \pm 0.03$ & $27.64 \pm 0.10$ & $26.66 \pm 0.04$ & $25.98 \pm 0.04$ \\
\hline $52768.414 \ldots \ldots \ldots \ldots \ldots \ldots$ & $27.81 \pm 0.11$ & $26.52 \pm 0.03$ & $26.02 \pm 0.04$ & $27.96 \pm 0.11$ & $27.27 \pm 0.08$ & $25.88 \pm 0.03$ \\
\hline 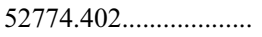 & $27.98 \pm 0.13$ & $26.59 \pm 0.06$ & $26.05 \pm 0.04$ & $28.27 \pm 0.17$ & $27.34 \pm 0.04$ & $25.80 \pm 0.04$ \\
\hline 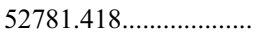 & $26.95 \pm 0.04$ & $26.68 \pm 0.04$ & $26.00 \pm 0.03$ & $28.67 \pm 0.23$ & $27.99 \pm 0.10$ & $25.72 \pm 0.03$ \\
\hline \multicolumn{7}{|c|}{ F814W } \\
\hline $52729.645 \ldots \ldots \ldots \ldots \ldots \ldots$ & $26.80 \pm 0.06$ & $25.72 \pm 0.04$ & $25.21 \pm 0.04$ & $26.50 \pm 0.05$ & $26.38 \pm 0.03$ & $24.92 \pm 0.02$ \\
\hline $52745.516 \ldots \ldots \ldots \ldots \ldots \ldots$ & $26.73 \pm 0.05$ & $26.23 \pm 0.05$ & $24.89 \pm 0.04$ & $27.06 \pm 0.08$ & $26.29 \pm 0.03$ & $25.15 \pm 0.03$ \\
\hline $52760.340 \ldots \ldots \ldots \ldots \ldots \ldots \ldots$ & $26.47 \pm 0.07$ & $26.08 \pm 0.07$ & $24.96 \pm 0.04$ & $26.49 \pm 0.05$ & $26.77 \pm 0.07$ & $25.04 \pm 0.03$ \\
\hline $52768.547 \ldots \ldots \ldots \ldots \ldots \ldots$ & $26.76 \pm 0.08$ & $25.72 \pm 0.04$ & $25.02 \pm 0.03$ & $26.71 \pm 0.05$ & $26.38 \pm 0.04$ & $24.93 \pm 0.03$ \\
\hline $52781.469 \ldots \ldots \ldots \ldots \ldots \ldots \ldots$ & $26.56 \pm 0.05$ & $25.79 \pm 0.04$ & $25.34 \pm 0.04$ & $26.35 \pm 0.04$ & $26.23 \pm 0.03$ & $24.76 \pm 0.02$ \\
\hline MJD & 3412. & 1842. & 79983 & 43163 & 5858. & 68212 \\
\hline \multicolumn{7}{|c|}{ F555W } \\
\hline $52729.512 \ldots$ & $27.65 \pm 0.08$ & $27.37 \pm 0.07$ & $27.42 \pm 0.06$ & $26.99 \pm 0.06$ & $27.19 \pm 0.05$ & $26.69 \pm 0.06$ \\
\hline $52737.582 \ldots \ldots \ldots \ldots \ldots \ldots . . . . . . . .$. & $27.96 \pm 0.11$ & $27.83 \pm 0.06$ & $27.23 \pm 0.05$ & $27.34 \pm 0.07$ & $27.41 \pm 0.06$ & $26.15 \pm 0.04$ \\
\hline 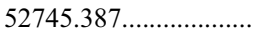 & $26.70 \pm 0.03$ & $27.18 \pm 0.05$ & $27.74 \pm 0.08$ & $27.53 \pm 0.08$ & $26.67 \pm 0.04$ & $26.20 \pm 0.05$ \\
\hline 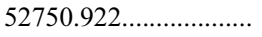 & $27.63 \pm 0.07$ & $27.49 \pm 0.07$ & $27.79 \pm 0.07$ & $26.82 \pm 0.03$ & $27.01 \pm 0.05$ & $26.71 \pm 0.06$ \\
\hline $52756.125 \ldots \ldots \ldots \ldots \ldots \ldots$ & $27.61 \pm 0.07$ & $27.64 \pm 0.08$ & $27.00 \pm 0.04$ & $27.09 \pm 0.06$ & $27.12 \pm 0.07$ & $26.82 \pm 0.07$ \\
\hline $52758.055 \ldots \ldots \ldots \ldots \ldots \ldots$ & $26.92 \pm 0.03$ & $27.81 \pm 0.08$ & $27.43 \pm 0.06$ & $27.39 \pm 0.08$ & $27.43 \pm 0.08$ & $27.13 \pm 0.09$ \\
\hline $52760.129 \ldots \ldots \ldots \ldots \ldots \ldots$ & $27.22 \pm 0.05$ & $27.91 \pm 0.11$ & $27.66 \pm 0.06$ & $27.27 \pm 0.07$ & $27.48 \pm 0.08$ & $27.08 \pm 0.07$ \\
\hline $52762.059 \ldots \ldots \ldots \ldots \ldots \ldots$ & $27.20 \pm 0.04$ & $28.00 \pm 0.15$ & $27.49 \pm 0.07$ & $27.57 \pm 0.07$ & $27.86 \pm 0.13$ & $26.97 \pm 0.10$ \\
\hline $52766.133 \ldots \ldots \ldots \ldots \ldots \ldots . .$. & $27.44 \pm 0.06$ & $27.77 \pm 0.10$ & $27.76 \pm 0.09$ & $27.67 \pm 0.10$ & $27.43 \pm 0.06$ & $27.21 \pm 0.10$ \\
\hline 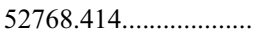 & $27.74 \pm 0.09$ & $27.41 \pm 0.05$ & $28.04 \pm 0.12$ & $27.48 \pm 0.09$ & $27.23 \pm 0.07$ & $26.93 \pm 0.08$ \\
\hline $52774.402 \ldots \ldots \ldots \ldots \ldots \ldots$ & $27.90 \pm 0.10$ & $27.23 \pm 0.04$ & $27.92 \pm 0.16$ & $27.63 \pm 0.08$ & $26.81 \pm 0.05$ & $26.17 \pm 0.04$ \\
\hline 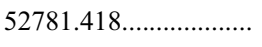 & $26.97 \pm 0.05$ & $27.23 \pm 0.06$ & $26.96 \pm 0.05$ & $26.67 \pm 0.05$ & $26.91 \pm 0.05$ & $26.31 \pm 0.05$ \\
\hline \multicolumn{7}{|c|}{ F814W } \\
\hline $52729.645 \ldots \ldots \ldots \ldots \ldots \ldots$ & $26.92 \pm 0.07$ & $26.62 \pm 0.06$ & $26.36 \pm 0.04$ & $26.28 \pm 0.04$ & $26.39 \pm 0.04$ & $25.53 \pm 0.05$ \\
\hline $52745.516 \ldots \ldots \ldots \ldots \ldots \ldots$ & $26.72 \pm 0.06$ & $26.26 \pm 0.05$ & $26.97 \pm 0.05$ & $26.04 \pm 0.03$ & $25.95 \pm 0.04$ & $25.50 \pm 0.05$ \\
\hline 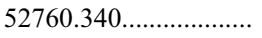 & $26.46 \pm 0.05$ & $26.72 \pm 0.08$ & $26.58 \pm 0.05$ & $26.31 \pm 0.04$ & $26.36 \pm 0.06$ & $25.96 \pm 0.05$ \\
\hline 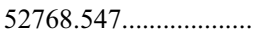 & $26.80 \pm 0.06$ & $26.21 \pm 0.05$ & $26.84 \pm 0.06$ & $26.59 \pm 0.06$ & $25.95 \pm 0.04$ & $25.46 \pm 0.04$ \\
\hline 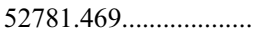 & $26.80 \pm 0.05$ & $26.28 \pm 0.06$ & $26.56 \pm 0.04$ & $26.07 \pm 0.03$ & $26.00 \pm 0.05$ & $25.53 \pm 0.05$ \\
\hline
\end{tabular}

NotE.-Table 5 is also available in machine-readable form in the electronic edition of the Astrophysical Journal.

${ }^{a}$ MJD is the Julian Date minus 2,400,000. 


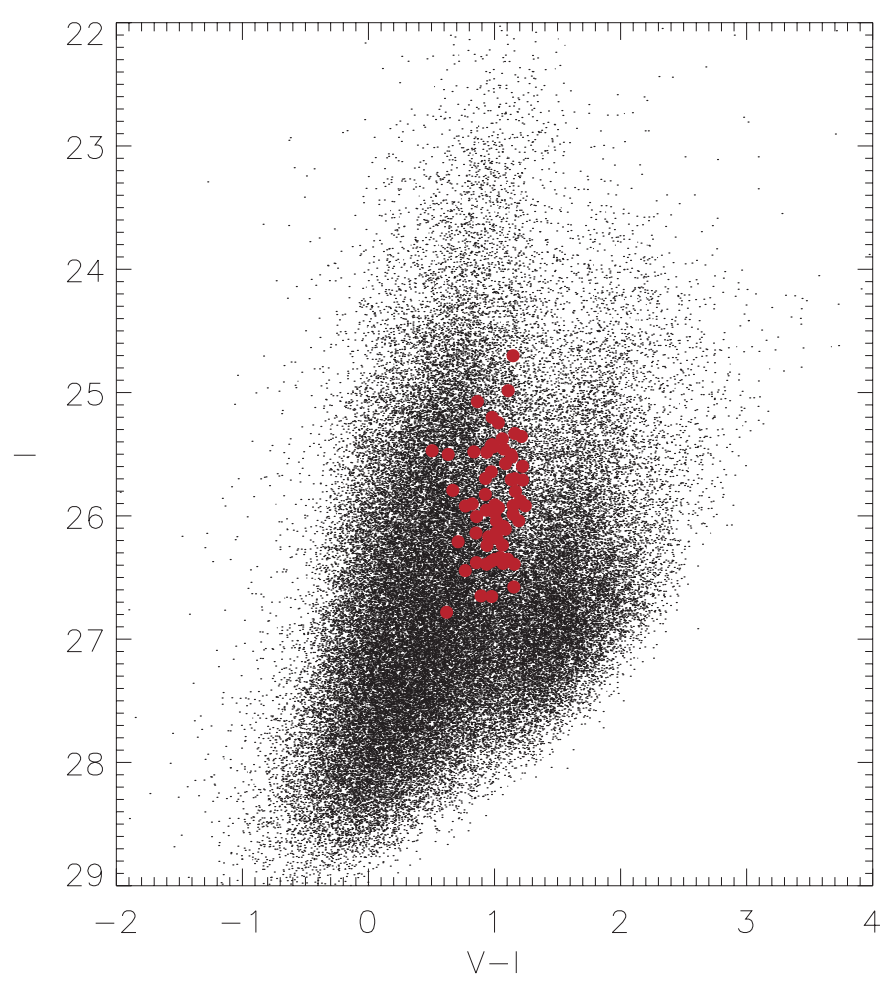

FIG. 6.-Color-magnitude diagram of all stars in the master catalog for NGC 3370. Large filled symbols show the positions of the Cepheids identified in NGC 3370, located as expected in the instability strip between the blue giants and red giants (post-main-sequence stars).

\subsection{Cepheid Distances to Other SN Ia Hosts}

In order to provide a consistent Cepheid-based distance to NGC 3982, the host of SN 1998aq (whose photometry is presented here for the first time in $\S 3$ ), we have revisited the analysis of the Cepheids by both the STS and SKP teams (Saha et al. 2001; Stetson \& Gibson 2001). The analyses of both teams are careful and thorough, and both provide similar tables of discovered Cepheids and their photometric parameters. Saha et al. (2001) conclude that $\mu_{0}=\mu_{w}=31.72 \pm 0.14 \mathrm{mag}$, while Stetson \& Gibson (2001) conclude that $\mu_{0}=\mu_{w}=31.56 \pm 0.08 \mathrm{mag}$ (considering only statistical uncertainties). As noted by Stetson \& Gibson (2001) and confirmed here, despite the independence of the analysis, the primary cause for the difference in distance modulus is from the choice of $P-L$ relations; Stetson \& Gibson (2001) use the same OGLE-based relation as F01, while Saha et al. (2001) use the earlier relation from MF91. Repeating the analysis of the Cepheids observed in NGC 3982 as reported by the two groups, but now using the $P-L$ relation from the OGLE+10 Cepheids (Thim et al. 2003), we find $\mu_{w}=31.60 \pm$ $0.08 \mathrm{mag}$.

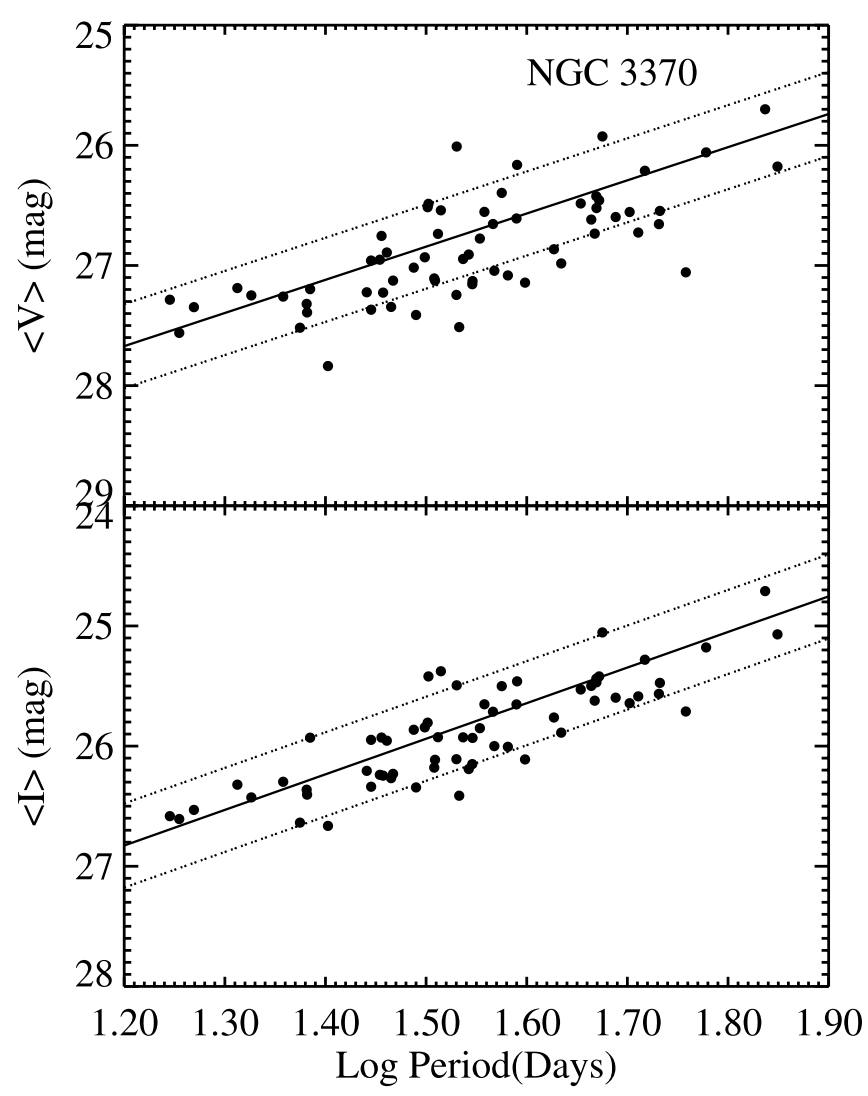

FIG. 7. $-V$-band and $I$-band $P-L$ relation for Cepheids identified by one of us (P. B. S.) in NGC 3370. The $P-L$ relation shown is from the OGLE+10 Cepheids (Thim et al. 2003) and the observed dispersion is 0.31 and $0.24 \mathrm{mag}$ for the $V$ and $I$ bands, respectively. An approximate instability strip width of $0.35 \mathrm{mag}$ is shown for comparison.

As previously discussed, there are two additional SNe Ia (SN $1990 \mathrm{~N}$ and SN 1981B) whose characteristics and data quality match the modern sample of high-quality objects defining the Hubble flow and thus can provide calibrations of the fiducial luminosity of SNe Ia as good as those from SN 1998aq and SN 1994ae. Cepheids in their hosts, NGC 4639 (SN 1990N) and NGC 4536 (SN 1981B), were observed by the STS collaboration and were analyzed by both STS (Saha et al. 1996, 1997) and SKP (Gibson et al. 2000). For consistency we have refitted the published photometry of these Cepheids to relations based on the OGLE+10 Cepheids as above. We used the photometry of Gibson et al. (2000) for NGC 4536, which, unlike that of Saha et al. (1996), finds consistency between the moduli from WFPC2 chip 2 and that from the other three chips. For NGC 4536 we find $\mu_{0}=30.78 \pm 0.07$ mag and for NGC 4639 we find $\mu_{0}=31.62 \pm 0.09 \mathrm{mag}$.

TABLE 6

Slopes and Zero Points for Cepheid $P$ - $L$ Relations

\begin{tabular}{|c|c|c|c|c|c|c|c|}
\hline Set & $a_{V}$ & $a_{I}$ & $a_{W}$ & $b_{V}$ & $b_{I}$ & $b_{W}$ & References \\
\hline OGLE ...................... & -2.760 & -2.962 & -3.255 & -1.458 & -1.942 & -2.644 & 1 \\
\hline MF91 ….................. & -2.760 & -3.060 & -3.495 & -1.400 & -1.810 & -2.405 & 2 \\
\hline OGLE $+10 \ldots \ldots \ldots \ldots$ & -2.480 & -2.820 & -3.313 & -1.750 & -2.090 & -2.583 & 3 \\
\hline
\end{tabular}

Notes.-Slopes and zero points for the $P$ - $L$ relations resulting from the four samples of Cepheids described in $\S 2.3$. The relations are defined by $M_{C}=a_{C} \log P+b_{C}$, with $M_{C}$ the absolute magnitude of a Cepheid in photometric band $C, P$ its period (days), and $a_{C}$ and $b_{C}$ the slope and zero point of the relation, respectively. $W$ represents the Wesenheit reddening-free index described in the text.

REFERENCES.-(1) F01; (2) MF91; (3) Thim et al. 2003. 


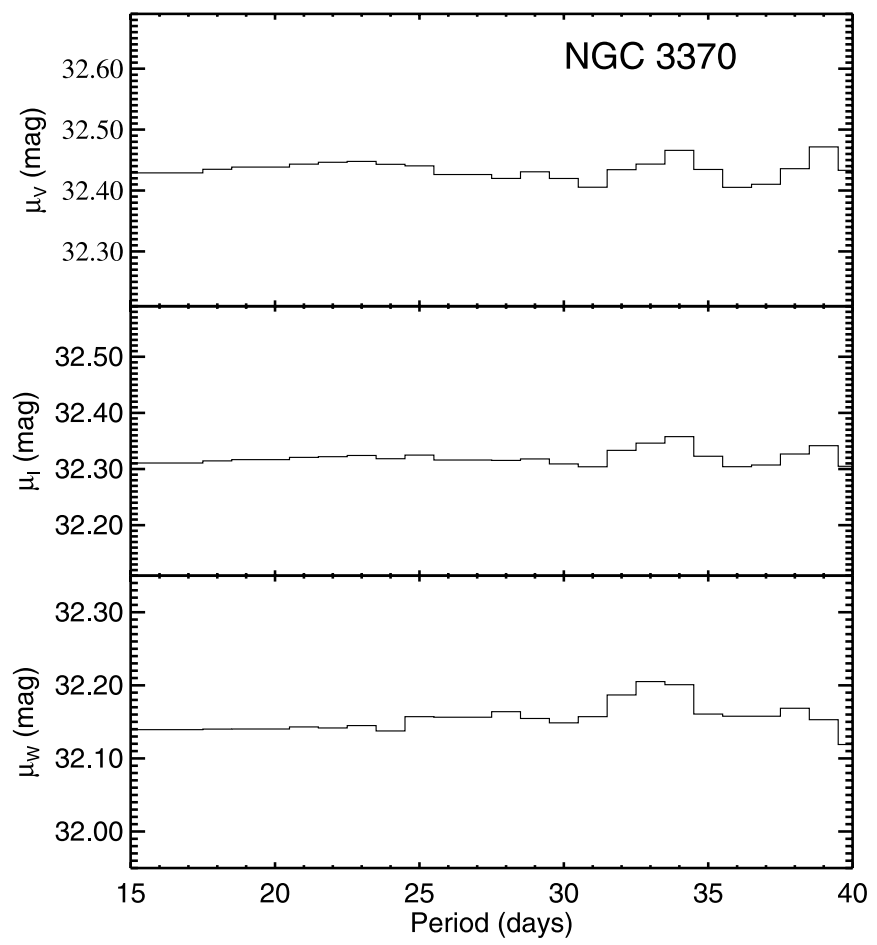

FIG. 8. $V$-band (top), I-band (middle), and reddening-free (bottom) distance modulus as a function of the lower period (magnitude limit) cutoff used. As shown, the distance moduli are relatively insensitive to the cutoff due to the strong detectability of all Cepheids with ACS at the faint end.

\subsection{Metallicity}

Mounting evidence indicates that there is a significant dependence of the apparent magnitudes of Cepheids (at fixed period) on the metallicity of the host galaxy. A marginal (1.5 $\sigma$ ) detection of this dependence by Kennicutt et al. (1998) was derived from two different Cepheid fields in M101 differing in $[\mathrm{O} / \mathrm{H}]$ by 0.7 dex. A greatly improved calibration of the dependence (consistent with that used by the Key Project) was derived by Sakai et al. (2004) using 17 Cepheid hosts with independent distances from the apparent magnitudes of the tip of the red giant branch. This calibration, $\delta(m-M) / \delta[\mathrm{O} / \mathrm{H}]=-0.24 \pm 0.05 \mathrm{mag}$ $\mathrm{dex}^{-1}$, is used here to correct the SN Ia host Cepheids for their metallicity difference with the LMC.

The metallicities of the two SN hosts, NGC 3370 and NGC 3982, are not available in the literature. To obtain estimates, we followed the procedure established by Zaritsky et al. (1994) and subsequently utilized by Kennicutt et al. (1998) and Sakai et al.

TABLE 7

Cepheid-Based Distances to NGC 3370

\begin{tabular}{|c|c|c|c|}
\hline \multirow[b]{2}{*}{ Zero PoInt } & \multicolumn{3}{|c|}{$P$ - $L$ Relations } \\
\hline & MF91 & OGLE & OGLE +10 \\
\hline \multicolumn{4}{|l|}{ P. B. S. selection: } \\
\hline 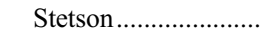 & $32.28 \pm 0.03$ & $32.13 \pm 0.03$ & $32.17 \pm 0.03$ \\
\hline Sirianni ............................ & $32.33 \pm 0.03$ & $32.19 \pm 0.03$ & $32.23 \pm 0.03$ \\
\hline \multicolumn{4}{|l|}{$\chi^{2}$ selection: } \\
\hline Stetson ............................. & $32.32 \pm 0.03$ & $32.18 \pm 0.03$ & $32.21 \pm 0.03$ \\
\hline Sirianni ............................. & $32.38 \pm 0.03$ & $32.24 \pm 0.03$ & $32.27 \pm 0.03$ \\
\hline \multicolumn{4}{|l|}{ Robust $\chi^{2}$ selection: } \\
\hline Stetson ............................. & $32.32 \pm 0.04$ & $32.17 \pm 0.04$ & $32.20 \pm 0.04$ \\
\hline Sirianni ............................. & $32.37 \pm 0.04$ & $32.23 \pm 0.04$ & $32.26 \pm 0.04$ \\
\hline
\end{tabular}

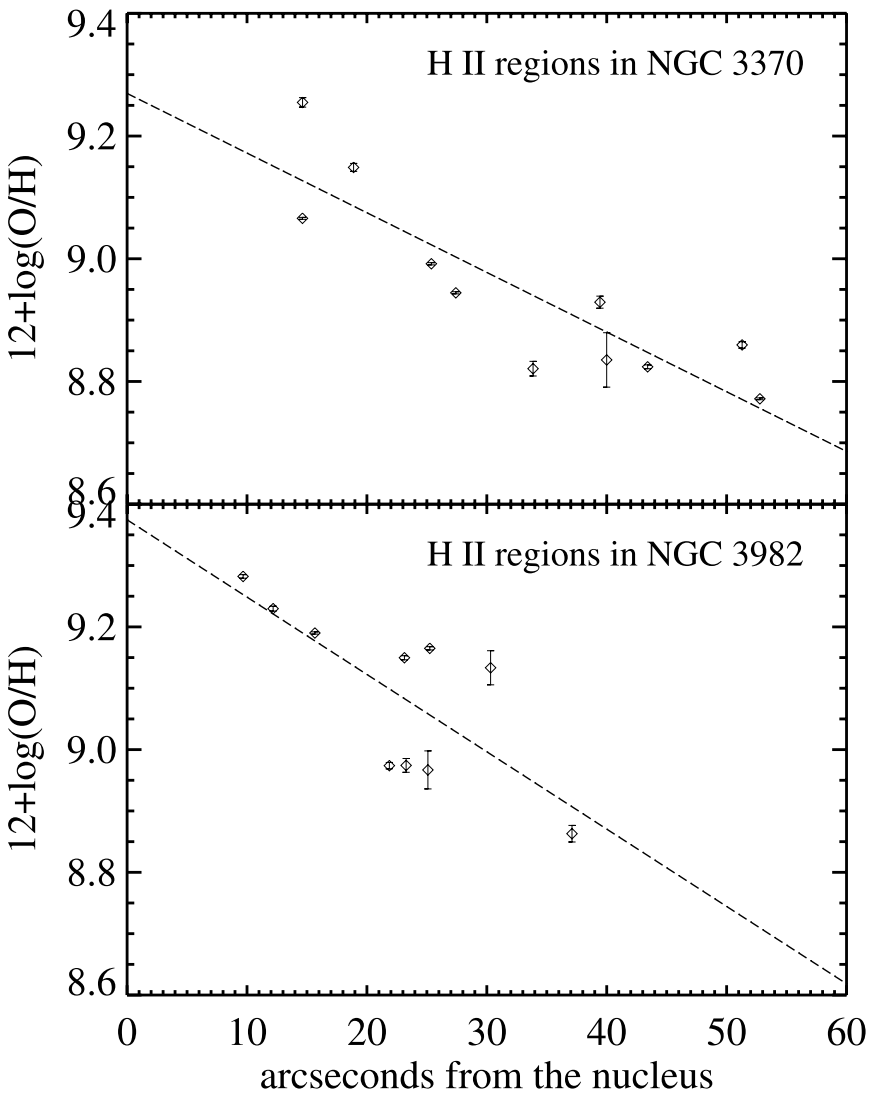

Fig. 9.-Oxygen-to-hydrogen ratios of $\mathrm{H}$ II regions in the host galaxies of two SNe Ia, NGC 3370 and NGC 3982. Following the method of Zaritsky et al. (1994), emission-line intensity ratios of [O II] $\lambda \lambda 3726,3729,[\mathrm{O}$ III] $\lambda \lambda 4959,5007$, and $\mathrm{H} \beta$ were used to derive values of $12+\log (\mathrm{O} / \mathrm{H})$ for $\mathrm{H}$ II regions. A linear gradient was fitted to determine the value of the metallicity correction at the characteristic radial position of the observed Cepheids, $50^{\prime \prime}$. The uncertainties shown are purely statistical; the dominant source of variance is clearly systematic.

(2004). Slit masks were produced for the Low Resolution Imaging Spectrometer (Oke et al. 1995) on the Keck I telescope for likely $\mathrm{H}$ II regions based on the archival HST and ground-based (Lick Observatory $1 \mathrm{~m}$ "Nickel telescope") images of the two galaxies observed through narrowband $\mathrm{H} \alpha$ and broadband filters. Emission-line intensity ratios of [O II] $\lambda \lambda 3726,3729$, [O III] $\lambda \lambda 4959,5007$, and $\mathrm{H} \beta$ were measured from our resulting spectroscopy of the $\mathrm{H}$ II regions. The line ratios were corrected for reddening as derived from the Balmer decrement (assuming the unreddened values from case B recombination; Osterbrock 1989). For each $\mathrm{H}$ II region, the $R_{23}$ value [defined as $([\mathrm{O} \mathrm{II}]+[\mathrm{O}$ III $\left.]) / \mathrm{H} \beta\right]$ and values for $12+\log (\mathrm{O} / \mathrm{H})$ were derived from the empirical calibration from Zaritsky et al. (1994), which assumes $[\mathrm{O} / \mathrm{H}]_{\text {solar }}=$ $7.9 \times 10^{-4}$. For NGC 3370 and NGC 3982, 10 and seven independent $\mathrm{H}$ II regions, respectively, were measured and their metallicities calibrated.

A strong, linear gradient in metallicity with distance from the nucleus of each host is seen, as expected, with a dispersion of $\sim 0.1 \mathrm{dex}$. The mean metallicity at the same average galactocentric radius as the HST-observed Cepheids of $50^{\prime \prime}$ for both galaxies was inferred from a linear fit to the gradients shown in Figure 9, the results of which are given in Table 8 .

\section{THE LIGHT CURVES OF SN 1994ae AND SN 1998aq}

SN 1994ae and SN 1998aq were both spectroscopically normal SNe Ia. SN 1994ae in NGC 3370 was discovered (Van Dyk et al. 1994) on 1994 November 3 (UT dates are used throughout 
TABLE 8

Metallicities of SN Ia Hosts at Cepheid Locations

\begin{tabular}{cccc}
\hline \hline Host & $12+\log (\mathrm{O} / \mathrm{H})$ & {$[\mathrm{O} / \mathrm{H}]-[\mathrm{O} / \mathrm{H}]_{\mathrm{LMC}}$} & $\begin{array}{c}\delta(m-M) \\
(\mathrm{mag})\end{array}$ \\
\hline NGC $3370 \ldots \ldots \ldots$. & $8.80 \pm 0.05$ & $0.30 \pm 0.05$ & $0.07 \pm 0.03$ \\
NGC $3982 \ldots \ldots \ldots \ldots$. & $8.75 \pm 0.05$ & $0.25 \pm 0.05$ & $0.06 \pm 0.03$ \\
NGC $4639^{\mathrm{a}} \ldots \ldots \ldots$. & $9.00 \pm 0.20$ & $0.50 \pm 0.20$ & $0.12 \pm 0.08$ \\
NGC $4536^{\mathrm{a}} \ldots \ldots \ldots$. & $8.85 \pm 0.20$ & $0.35 \pm 0.20$ & $0.08 \pm 0.05$ \\
\hline
\end{tabular}

${ }^{\text {a }}$ From Gibson et al. (2000).

this paper) by the Leuschner Observatory Supernova Search (Treffers et al. 1995) with the Berkeley Automatic Imaging Telescope (Treffers et al. 1992; Richmond et al. 1993). At that time, it was $\sim 12$ days before maximum brightness, earlier than nearly all previous SN Ia discoveries. Its location in the galaxy was 6".1 north and 29".7 west as seen in Figure 2. Photometric monitoring of the light curve commenced immediately by the CfA Survey I (Riess et al. 1999a). An identification spectrum was obtained by Iijima et al. (1994), and additional spectroscopy was obtained at the Mount Hopkins $1.5 \mathrm{~m}$ telescope (T. Matheson et al. 2005 , in preparation), revealing typical SN Ia features including strong, broad, blueshifted Si II and S II absorption.

SN 1998aq in NGC 3982 was discovered on 1998 April 13 by M. Armstrong during the course of the UK Nova/Supernova Patrol, 18" west and 7" north of the center of NGC 3982 (Hurst et al. 1998). The original CCD discovery images have been transformed to standard passband systems by Riess et al. (1999b). Photometric monitoring of the SN commenced immediately at the FLWO $1.2 \mathrm{~m}$ telescope by the CfA Survey II (Jha et al. 2005). An identification spectrum was obtained on April 15 by Ayani \& Yamaoka (1998), revealing broad, blueshifted absorption of Si II and $\mathrm{S}$ II consistent with a typical SN Ia. A comprehensive spectroscopic record of SN 1998aq was published by Branch et al. (2003).

The relative proximity of both of these $\mathrm{SNe}$ Ia $(d<30 \mathrm{Mpc}$ and $V_{\max } \leq 13.0 \mathrm{mag}$ ) provides two rare and valuable opportunities to improve the calibration of the fiducial luminosity of
SNe Ia using the techniques and tools of modern photometry. Both $\mathrm{SNe}$ are ideal calibrators by the criteria defined in $\S 1$.

Initial photometry was published for SN 1994ae by Riess et al. (1999a), but this reduction lacked the advantages of galaxy template subtraction and repeated zero-point calibrations. Here we have undertaken an improved calibration of SN 1994ae by performing galaxy subtraction and repeating the calibration of the field stars 4 times. For SN 1998aq, we present the photometry here for the first time.

For SN 1994ae, photometric monitoring was conducted with the FLWO $1.2 \mathrm{~m}$ telescope, an $f / 8$ Ritchey-Chrétien reflector equipped with a thick front-illuminated Loral CCD and a set of Johnson $U B V$ and Kron-Cousins RI passbands (hereafter the "SAO" filter set). Further details of the equipment, typical color terms, and broadband transmission functions are provided by Riess et al. (1999a), and updates to the equipment at this facility are described by Jha et al. (2005). On nights that were judged to be photometric, we performed all-sky photometry and derived a transformation from our detector measurements to the standard photometric conventions using Landolt (1992) standard stars. From each useful transformation we derived the magnitudes of comparison stars in the field of the supernova. For SN 1994ae, three photometric transformations (1995 January 7, 1999 December 8, and 2000 February 15) were derived from the $1.2 \mathrm{~m}$ telescope, and two more (2003 May 31 and 2003 June 1) came from the Lick Observatory $1 \mathrm{~m}$ Nickel telescope (for average color terms see Ho et al. 2001). Average magnitudes for field stars are given in Table 9, and a finder chart for these stars is shown in Figure 10. Galaxy templates were selected from the FLWO imaging on 1999 December 8.

In general, the same procedures as described for SN 1994ae were followed for SN 1998aq. Photometric monitoring was conducted with the FLWO $1.2 \mathrm{~m}$ telescope using the same SAO filter set as for SN 1994ae. During this monitoring, one of two thinned, back-side-illuminated, antireflection-coated Loral CCD detectors was used, primarily the "4Shooter" and on two dates the "AndyCam" (MJD -2, 450, $000=960$ and 1013); for more detailed specifications for these cameras see Jha et al. (2005).

TABLE 9

Comparison Stars for SN 1994ae

\begin{tabular}{|c|c|c|c|c|c|c|c|c|c|c|}
\hline Star & $U$ & $N$ & $B$ & $N$ & V & $N$ & $R$ & $N$ & $I$ & $N$ \\
\hline $01 \ldots \ldots \ldots \ldots \ldots \ldots$ & 18.214 & 1 & $18.402 \pm 0.022$ & 4 & $17.811 \pm 0.033$ & 3 & $17.443 \pm 0.045$ & 2 & $17.027 \pm 0.064$ & 3 \\
\hline $02 \ldots$ & 16.739 & 1 & $15.980 \pm 0.016$ & 4 & $15.062 \pm 0.023$ & 3 & $14.496 \pm 0.001$ & 2 & $14.045 \pm 0.011$ & 3 \\
\hline $03 \ldots \ldots \ldots \ldots \ldots$ & 18.835 & 1 & $18.630 \pm 0.025$ & 4 & $17.924 \pm 0.025$ & 3 & $17.486 \pm 0.018$ & 2 & $17.059 \pm 0.069$ & 3 \\
\hline 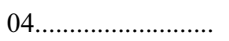 & 17.138 & 1 & $17.029 \pm 0.055$ & 3 & $16.380 \pm 0.013$ & 2 & 15.983 & 1 & $15.552 \pm 0.023$ & 2 \\
\hline 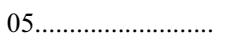 & $\ldots$ & $\ldots$ & $17.296 \pm 0.026$ & 3 & $16.717 \pm 0.008$ & 2 & 16.375 & 1 & $15.956 \pm 0.011$ & 2 \\
\hline 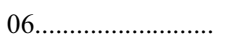 & 18.283 & 1 & $17.532 \pm 0.008$ & 2 & 16.530 & 1 & $\ldots$ & $\ldots$ & 15.247 & 1 \\
\hline 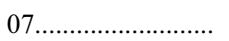 & 17.122 & 1 & $16.962 \pm 0.011$ & 2 & 16.308 & 1 & $\ldots$ & $\ldots$ & 15.440 & 1 \\
\hline 08 & $\ldots$ & $\ldots$ & $17.278 \pm 0.041$ & 2 & 16.562 & 1 & $\ldots$ & $\ldots$ & 15.682 & 1 \\
\hline 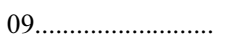 & 17.045 & 1 & $16.542 \pm 0.031$ & 2 & 15.743 & 1 & $\ldots$ & $\ldots$ & 14.830 & 1 \\
\hline $10 \ldots \ldots \ldots \ldots \ldots \ldots$ & $\ldots$ & $\ldots$ & $18.655 \pm 0.014$ & 2 & 17.322 & 1 & $\ldots$ & $\ldots$ & 15.688 & 1 \\
\hline 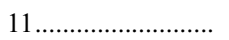 & $\ldots$ & $\ldots$ & 19.023 & 1 & 17.535 & 1 & $\ldots$ & $\ldots$ & 15.582 & 1 \\
\hline $12 \ldots \ldots \ldots \ldots \ldots \ldots \ldots$ & 15.845 & 1 & $15.370 \pm 0.023$ & 2 & 14.571 & 1 & $\ldots$ & $\ldots$ & 13.589 & 1 \\
\hline 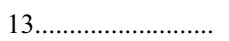 & 18.545 & 1 & $17.790 \pm 0.022$ & 2 & 16.848 & 1 & $\ldots$ & $\ldots$ & 15.672 & 1 \\
\hline 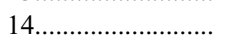 & 17.340 & 1 & $17.161 \pm 0.022$ & 2 & 16.485 & 1 & $\ldots$ & $\ldots$ & 15.597 & 1 \\
\hline $15 \ldots \ldots \ldots \ldots \ldots \ldots \ldots \ldots \ldots$ & 15.034 & 1 & $15.033 \pm 0.001$ & 2 & 14.516 & 1 & $\ldots$ & $\ldots$ & 13.764 & 1 \\
\hline 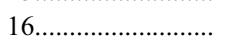 & $\ldots$ & $\ldots$ & 18.869 & 1 & 17.401 & 1 & $\ldots$ & $\ldots$ & 14.947 & 1 \\
\hline 17 & $\ldots$ & $\ldots$ & 19.130 & 1 & 17.601 & 1 & $\ldots$ & $\ldots$ & 14.880 & 1 \\
\hline 18 & $\ldots$ & $\ldots$ & $18.409 \pm 0.032$ & 2 & 17.677 & 1 & $\ldots$ & $\ldots$ & 16.792 & 1 \\
\hline 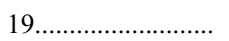 & 16.667 & 1 & $16.763 \pm 0.024$ & 2 & 16.268 & 1 & $\ldots$ & $\ldots$ & 15.593 & 1 \\
\hline 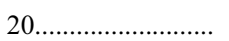 & $\ldots$ & $\ldots$ & 19.931 & 1 & 18.370 & 1 & $\ldots$ & $\ldots$ & 15.757 & 1 \\
\hline
\end{tabular}




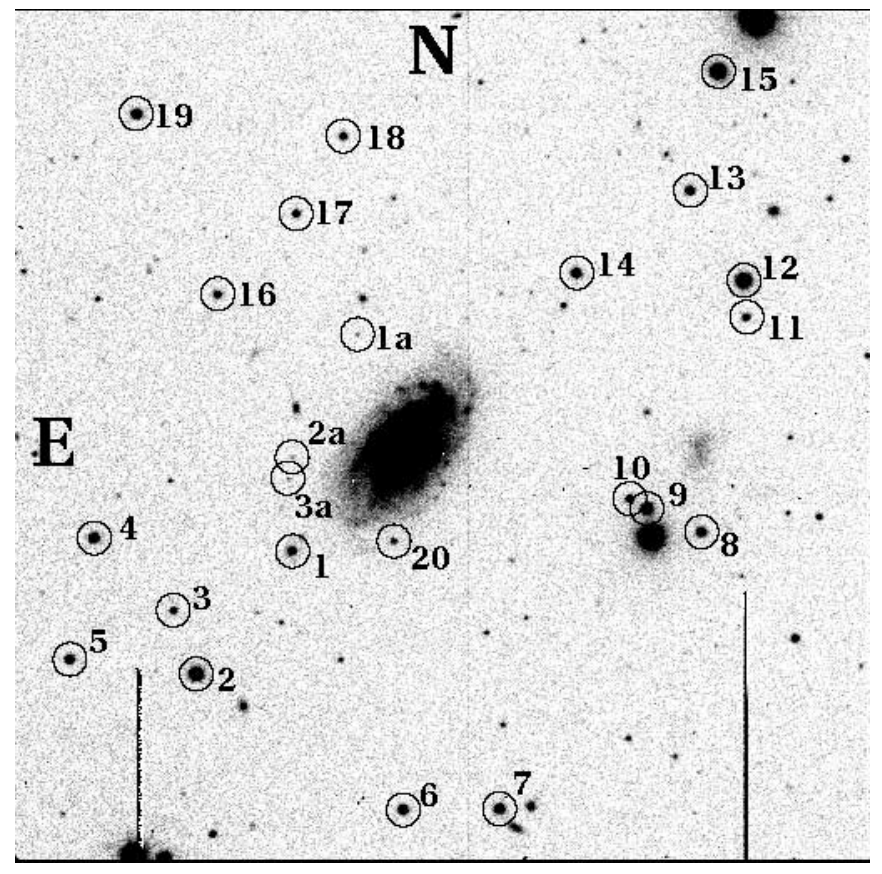

FIG. 10.-Ground-based comparison stars in the field of NGC 3370 used to calibrate the light curves of SN 1994ae. Image is from the $1.2 \mathrm{~m}$ telescope at the FLWO.

Average magnitudes for field stars are given in Table 10, and a finder chart for these stars is shown in Figure 11. For SN 1998aq, five independent transformations were used from the nights of 2003 May 31 (Nickel), 1998 June 15 and 22 (FLWO), and 2001 March 19 and 26 (FLWO). Galaxy templates were obtained from imaging at the FLWO on 2000 November 27.

Supernova magnitudes were determined from the relative photometry of the comparison stars and the SN after galaxy subtraction. The PSF fitting procedure in IRAF was used to conduct the photometry. Model PSFs were constructed for each image using the bright, isolated stars in the frame and were then used to fit the magnitudes of the $\mathrm{SN}$ and the comparison stars. Average color terms (given in Riess et al. 1999a; Jha et al. 2005) were used to account for color differences between the $\mathrm{SN}$ and comparison stars and to transform the $\mathrm{SN}$ magnitudes to the

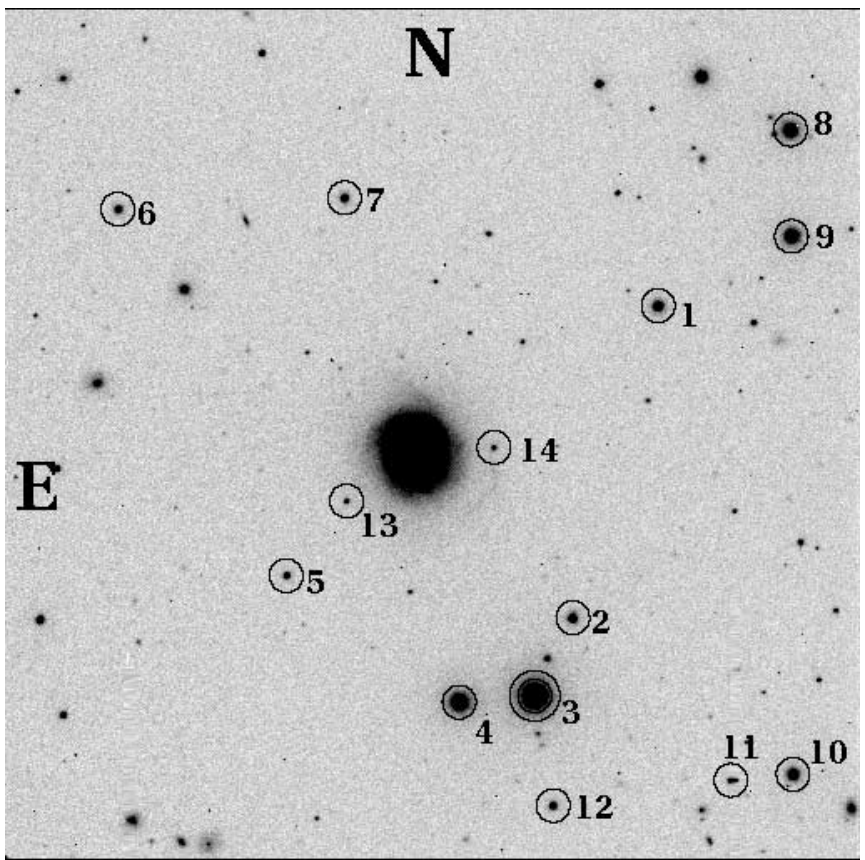

FIG. 11.-Ground-based comparison stars in the field of NGC 3982 used to calibrate the light curves of SN 1998aq. Image is from the $1.2 \mathrm{~m}$ telescope at the FLWO.

Johnson $U B V$ and Kron-Cousins $R I$ systems. ${ }^{9}$ The final photometry for SN 1994ae and SN 1998aq is listed in Tables 11 and 12, respectively, and shown in Figure 12. The uncertainty of the measurements was determined as the quadrature sum of the individual PSF fit uncertainty and the standard deviation of the transformation from the comparison stars.

A useful cross-check of the consistency of our photometry would be to compare the magnitudes of comparison stars in the

\footnotetext{
${ }^{9}$ A more detailed accounting for the effect of the nonstellar spectral energy distribution of SNe Ia on transformed magnitudes (i.e., "S-corrections"; Suntzeff et al. 1999) is easily accomplished by returning the magnitudes to the natural system (using the average color terms) and making use of the transmission functions in Riess et al. (1999a) and Jha et al. (2005) in addition to template SN Ia spectra to transform the data to other passband conventions.
}

TABLE 10

COMPARISON STARS FOR SN 1998aq

\begin{tabular}{|c|c|c|c|c|c|c|c|c|c|c|}
\hline Star & $U$ & $N$ & $B$ & $N$ & $V$ & $N$ & $R$ & $N$ & $I$ & $N$ \\
\hline 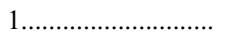 & $17.337 \pm 0.005$ & 3 & $16.180 \pm 0.027$ & 4 & $14.926 \pm 0.025$ & 4 & $14.126 \pm 0.017$ & 4 & $13.424 \pm 0.029$ & 4 \\
\hline 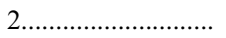 & $16.130 \pm 0.037$ & 4 & $16.093 \pm 0.030$ & 4 & $15.450 \pm 0.020$ & 4 & $15.077 \pm 0.018$ & 4 & $14.716 \pm 0.030$ & 4 \\
\hline ............... & $12.736 \pm 0.005$ & 2 & $12.761 \pm 0.016$ & 2 & $12.202 \pm 0.011$ & 2 & $11.874 \pm 0.010$ & 2 & $11.562 \pm 0.005$ & 2 \\
\hline . & $14.880 \pm 0.011$ & 2 & $14.200 \pm 0.012$ & 2 & $13.302 \pm 0.018$ & 2 & $12.782 \pm 0.018$ & 2 & $12.357 \pm 0.004$ & 2 \\
\hline ............... & $16.954 \pm 0.010$ & 4 & $17.079 \pm 0.046$ & 4 & $16.529 \pm 0.027$ & 4 & $16.196 \pm 0.040$ & 4 & $15.864 \pm 0.051$ & 4 \\
\hline 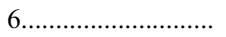 & $\ldots$ & $\ldots$ & $16.812 \pm 0.039$ & 3 & $15.926 \pm 0.044$ & 3 & $15.382 \pm 0.039$ & 3 & $14.924 \pm 0.034$ & 3 \\
\hline 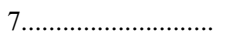 & $17.604 \pm 0.041$ & 3 & $16.728 \pm 0.037$ & 4 & $15.770 \pm 0.038$ & 4 & $15.196 \pm 0.035$ & 4 & $14.739 \pm 0.036$ & 4 \\
\hline 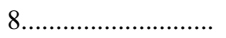 & $14.789 \pm 0.033$ & 2 & $14.590 \pm 0.002$ & 2 & $13.910 \pm 0.012$ & 2 & $13.510 \pm 0.004$ & 2 & $13.153 \pm 0.002$ & 2 \\
\hline 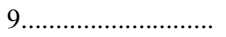 & $14.251 \pm 0.023$ & 2 & $14.345 \pm 0.002$ & 2 & $13.946 \pm 0.008$ & 2 & $13.700 \pm 0.010$ & 2 & $13.451 \pm 0.008$ & 2 \\
\hline 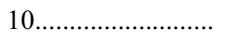 & $16.055 \pm 0.017$ & 3 & $15.597 \pm 0.018$ & 3 & $14.780 \pm 0.015$ & 3 & $14.310 \pm 0.010$ & 3 & $13.896 \pm 0.006$ & 3 \\
\hline 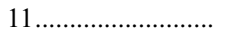 & $\ldots$ & $\ldots$ & $17.825 \pm 0.019$ & 2 & $17.060 \pm 0.000$ & 2 & $16.589 \pm 0.008$ & 2 & $16.094 \pm 0.015$ & 2 \\
\hline 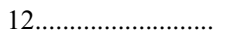 & $\ldots$ & $\ldots$ & $17.069 \pm 0.024$ & 3 & $16.134 \pm 0.019$ & 3 & $15.572 \pm 0.008$ & 3 & $15.053 \pm 0.017$ & 3 \\
\hline $13 \ldots \ldots \ldots \ldots \ldots \ldots \ldots \ldots \ldots \ldots \ldots \ldots \ldots \ldots$ & $\ldots$ & $\ldots$ & $19.154 \pm 0.017$ & 4 & $17.779 \pm 0.034$ & 5 & $16.878 \pm 0.050$ & 5 & $15.919 \pm 0.029$ & 5 \\
\hline 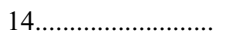 & $\ldots$ & $\ldots$ & $19.124 \pm 0.045$ & 5 & $17.767 \pm 0.036$ & 5 & $16.903 \pm 0.026$ & 5 & $16.140 \pm 0.032$ & 5 \\
\hline
\end{tabular}


TABLE 11

Photometry of SN 1994ae

\begin{tabular}{|c|c|c|c|c|c|}
\hline $\mathrm{MJD}^{\mathrm{a}}$ & $U$ & $B$ & $V$ & $R$ & $I$ \\
\hline $9672.97 \ldots \ldots \ldots \ldots \ldots \ldots$ & $\ldots$ & $14.865 \pm 0.041$ & $14.833 \pm 0.027$ & $14.711 \pm 0.022$ & $14.599 \pm 0.028$ \\
\hline $9674.96 \ldots \ldots \ldots \ldots \ldots \ldots$ & $\ldots$ & $14.109 \pm 0.044$ & $14.133 \pm 0.040$ & $14.007 \pm 0.026$ & $13.948 \pm 0.019$ \\
\hline 9676.96..................... & $\ldots$ & $13.730 \pm 0.167$ & $13.690 \pm 0.051$ & $13.580 \pm 0.019$ & $13.590 \pm 0.033$ \\
\hline $9678.00 \ldots \ldots \ldots \ldots \ldots \ldots$ & $\ldots$ & $13.516 \pm 0.030$ & $13.513 \pm 0.039$ & $13.422 \pm 0.018$ & $\ldots$ \\
\hline 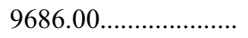 & $12.548 \pm 0.084$ & $13.059 \pm 0.024$ & $13.045 \pm 0.030$ & $13.046 \pm 0.028$ & $13.287 \pm 0.039$ \\
\hline $9686.99 \ldots \ldots \ldots \ldots \ldots \ldots$ & $12.608 \pm 0.044$ & $13.082 \pm 0.028$ & $13.077 \pm 0.021$ & $13.064 \pm 0.018$ & $13.322 \pm 0.031$ \\
\hline $9688.04 \ldots \ldots$ & $\ldots$ & $13.104 \pm 0.035$ & $13.118 \pm 0.030$ & $13.097 \pm 0.015$ & $13.363 \pm 0.042$ \\
\hline 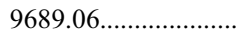 & $12.728 \pm 0.052$ & $13.190 \pm 0.047$ & $13.120 \pm 0.024$ & $13.097 \pm 0.100$ & $13.383 \pm 0.070$ \\
\hline $9690.03 \ldots \ldots \ldots \ldots \ldots \ldots$ & $12.753 \pm 0.052$ & $13.231 \pm 0.040$ & $13.141 \pm 0.036$ & $13.133 \pm 0.034$ & $13.449 \pm 0.083$ \\
\hline $9694.03 \ldots \ldots \ldots \ldots \ldots \ldots . . . . . . .$. & $\ldots$ & $13.435 \pm 0.038$ & $13.306 \pm 0.027$ & $13.312 \pm 0.023$ & $13.683 \pm 0.026$ \\
\hline $9695.03 \ldots \ldots \ldots \ldots \ldots \ldots . . . . . . . .$. & $\ldots$ & $13.515 \pm 0.031$ & $13.329 \pm 0.034$ & $13.358 \pm 0.020$ & $13.784 \pm 0.099$ \\
\hline $9695.97 \ldots \ldots \ldots \ldots \ldots \ldots$ & $\ldots$ & $13.576 \pm 0.035$ & $13.398 \pm 0.026$ & $13.438 \pm 0.013$ & $13.812 \pm 0.022$ \\
\hline $9696.96 \ldots \ldots \ldots \ldots \ldots \ldots$ & $\ldots$ & $13.674 \pm 0.028$ & $13.438 \pm 0.028$ & $13.525 \pm 0.015$ & $13.877 \pm 0.023$ \\
\hline 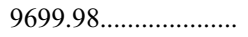 & $\ldots$ & $13.931 \pm 0.046$ & $13.645 \pm 0.023$ & $13.715 \pm 0.015$ & $13.957 \pm 0.031$ \\
\hline 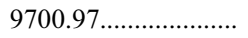 & $\ldots$ & $14.048 \pm 0.069$ & $13.673 \pm 0.024$ & $13.763 \pm 0.023$ & $14.001 \pm 0.031$ \\
\hline 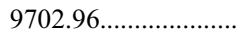 & $\ldots$ & $14.260 \pm 0.034$ & $13.785 \pm 0.022$ & $13.793 \pm 0.018$ & $13.943 \pm 0.030$ \\
\hline 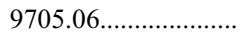 & $\ldots$ & $14.446 \pm 0.047$ & $13.888 \pm 0.028$ & $13.809 \pm 0.020$ & $13.890 \pm 0.024$ \\
\hline 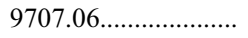 & $\ldots$ & $14.669 \pm 0.040$ & $13.960 \pm 0.040$ & $13.836 \pm 0.030$ & $13.886 \pm 0.060$ \\
\hline $9715.93 \ldots \ldots \ldots \ldots \ldots \ldots$ & $\ldots$ & $15.491 \pm 0.042$ & $14.418 \pm 0.029$ & $14.073 \pm 0.040$ & $13.773 \pm 0.070$ \\
\hline $9721.03 \ldots \ldots \ldots \ldots \ldots \ldots$ & $\ldots$ & $15.790 \pm 0.040$ & $14.677 \pm 0.059$ & $14.382 \pm 0.020$ & $13.998 \pm 0.036$ \\
\hline $9725.01 \ldots \ldots \ldots \ldots \ldots \ldots$ & $\ldots$ & $15.949 \pm 0.036$ & $14.942 \pm 0.022$ & $14.636 \pm 0.019$ & $14.256 \pm 0.024$ \\
\hline $9726.96 \ldots \ldots \ldots \ldots \ldots \ldots$ & $16.113 \pm 0.196$ & $15.996 \pm 0.060$ & $14.967 \pm 0.018$ & $14.685 \pm 0.020$ & $14.390 \pm 0.027$ \\
\hline $9740.86 \ldots \ldots \ldots \ldots \ldots \ldots$ & $\ldots$ & $16.262 \pm 0.042$ & $15.447 \pm 0.030$ & $15.249 \pm 0.035$ & $15.048 \pm 0.030$ \\
\hline 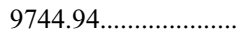 & $\ldots$ & $16.340 \pm 0.041$ & $15.541 \pm 0.032$ & $15.342 \pm 0.022$ & $15.202 \pm 0.032$ \\
\hline $9745.92 \ldots \ldots \ldots \ldots \ldots \ldots$ & $\ldots$ & $16.348 \pm 0.050$ & $15.589 \pm 0.026$ & $15.378 \pm 0.023$ & $15.263 \pm 0.033$ \\
\hline 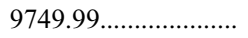 & $\ldots$ & $16.381 \pm 0.042$ & $15.650 \pm 0.052$ & $\ldots$ & $\ldots$ \\
\hline $9769.74 \ldots \ldots \ldots \ldots \ldots . . . . . . . .$. & $\ldots$ & $16.645 \pm 0.070$ & $16.191 \pm 0.058$ & $16.136 \pm 0.063$ & $16.214 \pm 0.070$ \\
\hline $9783.86 \ldots \ldots \ldots \ldots \ldots . . . . . . . .$. & $\ldots$ & $16.861 \pm 0.063$ & $16.541 \pm 0.069$ & $16.563 \pm 0.072$ & $\ldots$ \\
\hline 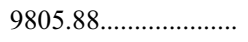 & $\ldots$ & $17.235 \pm 0.082$ & $16.982 \pm 0.083$ & $17.186 \pm 0.071$ & $17.362 \pm 0.080$ \\
\hline
\end{tabular}

Noте--Table 11 is also available in machine-readable form in the electronic edition of the Astrophysical Journal.

${ }^{a}$ MJD is the Julian Date minus 2,440,000.

SN fields as determined with the HST data and with the groundbased data. However, the dramatic difference in the sensitivity between the ground and HST results in a lack of stars that are well detected but not saturated in either of the observations. For our HST images, stars with $V<19.75 \mathrm{mag}$ and $I<19.5 \mathrm{mag}$ are saturated, but stars fainter than these limits are poorly detected (if at all) in the SN follow-up images obtained from the ground. In order to produce a comparison, we combined all of the deep follow-up imaging from the ground to increase the sensitivity. Consequently, three stars in the NGC 3370 field (labeled as stars 1a, 2a, and 3a) can be compared. For these three stars the mean difference in the $V$ band is $0.02 \pm 0.04 \mathrm{mag}$ (ground brighter), in good agreement. In the $I$ band, the only star that is not saturated is star 3a, whose ground-based magnitude is $0.04 \pm$ 0.17 mag brighter than measured by $H S T$, in good agreement but not of useful precision. No unsaturated stars in the NGC 3982 field are visible in the HST images.

The optical light curves of the other ideal calibrators, SN $1990 \mathrm{~N}$ and SN 1981B, are taken from Lira et al. (1998) and Buta \& Turner (1983), respectively.

\section{MEASURING THE HUBBLE CONSTANT}

\subsection{Light-Curve Fitting and Distance Calibrations}

$\mathrm{SNe}$ Ia are the most precise distance indicators known to delineate the global Hubble flow. Methods that utilize the relationship between luminosity, light curves, and colors achieve relative individual distance precision of $7 \%-8 \%$. With independent measurements of the distance to a few SN Ia hosts from
Cepheids or other means, we can establish an absolute distance scale for SNe Ia and, as a result, determine the Hubble constant.

Here we employ two popular methods for measuring SN Ia distances from their light curves, the "multicolor light-curve shape" (MLCS) method (Riess et al. 1996a) and a template fitting method [ $\Delta m_{15}(B)$; Phillips 1993]. In both cases (and equivalently applicable to other light-curve relations), we use the Cepheid-based distance measurements to the hosts of SN 1994ae and SN 1998aq to set each method's fiducial luminosity (i.e., zero point).

The MLCS method has been revised (and hereafter referred to as MLCS2k2) by Jha (2002) and S. Jha et al. (2005, in preparation) to include $U$-band light curves from Jha et al. (2005), a more self-consistent treatment of extinction, and an improved determination of the unreddened SN Ia color. In MLCS2k2 the light curves are empirically described in any bandpass $X$ as a superposition of time-dependent vectors in magnitudes:

$$
\boldsymbol{m}_{X}\left(t-t_{0}\right)=\boldsymbol{M}_{X}^{0}+\boldsymbol{\mu}_{0}+\eta_{X} A_{V}^{0}+\boldsymbol{P}_{X} \Delta+\boldsymbol{Q}_{X} \Delta^{2},
$$

where $\boldsymbol{m}_{X}\left(t-t_{0}\right)$ is the apparent magnitude light curve since a fiducial point in time $t_{0}$ (taken to be the time of $B$-band peak), $\boldsymbol{M}_{X}^{0}$ is the absolute magnitude light curve of the fiducial SN Ia, $\mu_{0}$ is the true distance modulus, $A_{V}^{0}$ is the visual extinction at $t_{0}, \boldsymbol{\eta}_{X}$ contains the time-dependent reddening law (see S. Jha et al. 2005, in preparation), $\Delta$ is the luminosity correction corresponding to $M_{V}\left(t_{0}\right)-M_{V}^{0}\left(t_{0}\right)$, and $\boldsymbol{P}_{X}$ and $\boldsymbol{Q}_{X}$ are vectors describing the change in light-curve shape with luminosity. After determining the form of $\boldsymbol{P}_{X}$ and $\boldsymbol{Q}_{X}$ from a Hubble flow training 
TABLE 12

РнотометRY of SN 1998aq

\begin{tabular}{|c|c|c|c|c|c|}
\hline $\mathrm{MJD}^{\mathrm{a}}$ & $U$ & $B$ & $V$ & $R$ & $I$ \\
\hline $920.64500 \ldots$. & $12.724 \pm 0.017$ & $13.551 \pm 0.027$ & $13.708 \pm 0.019$ & $13.613 \pm 0.017$ & $13.607 \pm 0.015$ \\
\hline 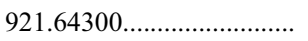 & $12.414 \pm 0.016$ & $13.264 \pm 0.022$ & $13.431 \pm 0.016$ & $13.328 \pm 0.016$ & $13.359 \pm 0.024$ \\
\hline 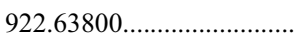 & $12.202 \pm 0.016$ & $13.046 \pm 0.022$ & $13.208 \pm 0.012$ & $13.110 \pm 0.011$ & $13.160 \pm 0.019$ \\
\hline 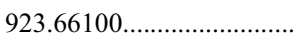 & $12.030 \pm 0.020$ & $12.861 \pm 0.019$ & $13.036 \pm 0.013$ & $12.930 \pm 0.013$ & $13.010 \pm 0.014$ \\
\hline 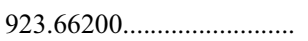 & $12.031 \pm 0.026$ & $12.867 \pm 0.020$ & $13.029 \pm 0.014$ & $12.929 \pm 0.016$ & $13.005 \pm 0.021$ \\
\hline 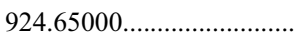 & $11.921 \pm 0.015$ & $12.729 \pm 0.021$ & $12.881 \pm 0.018$ & $12.788 \pm 0.014$ & $12.879 \pm 0.024$ \\
\hline $924.65100 \ldots \ldots \ldots \ldots$ & $11.916 \pm 0.019$ & $12.718 \pm 0.030$ & $12.885 \pm 0.019$ & $12.790 \pm 0.014$ & $12.874 \pm 0.026$ \\
\hline 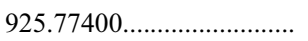 & $11.812 \pm 0.014$ & $12.600 \pm 0.030$ & $12.766 \pm 0.025$ & $12.670 \pm 0.026$ & $12.776 \pm 0.023$ \\
\hline $925.77700 \ldots \ldots \ldots \ldots \ldots \ldots \ldots . .$. & $11.816 \pm 0.010$ & $12.605 \pm 0.019$ & $12.777 \pm 0.015$ & $12.661 \pm 0.027$ & $12.774 \pm 0.026$ \\
\hline $925.78000 \ldots \ldots \ldots \ldots \ldots \ldots . .$. & $\ldots$ & $\ldots$ & $12.752 \pm 0.023$ & $\ldots$ & $\ldots$ \\
\hline 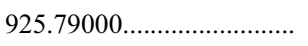 & $\ldots$ & $\ldots$ & $12.754 \pm 0.028$ & $\ldots$ & $\ldots$ \\
\hline 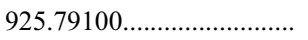 & $\ldots$ & $12.596 \pm 0.043$ & $12.763 \pm 0.030$ & $12.661 \pm 0.021$ & $12.775 \pm 0.017$ \\
\hline 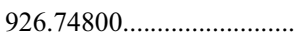 & $11.732 \pm 0.065$ & $12.523 \pm 0.047$ & $12.666 \pm 0.025$ & $12.602 \pm 0.019$ & $12.737 \pm 0.022$ \\
\hline 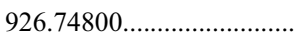 & $11.745 \pm 0.049$ & $12.522 \pm 0.043$ & $12.666 \pm 0.020$ & $12.615 \pm 0.051$ & $12.745 \pm 0.022$ \\
\hline $928.75500 \ldots \ldots \ldots \ldots \ldots \ldots \ldots \ldots \ldots$ & $11.715 \pm 0.015$ & $12.419 \pm 0.027$ & $12.531 \pm 0.019$ & $12.501 \pm 0.019$ & $12.699 \pm 0.024$ \\
\hline $928.75500 \ldots \ldots \ldots \ldots \ldots .$. & $11.704 \pm 0.032$ & $12.410 \pm 0.029$ & $12.537 \pm 0.022$ & $12.486 \pm 0.025$ & $12.701 \pm 0.019$ \\
\hline $930.77000 \ldots \ldots \ldots \ldots \ldots . .$. & $11.723 \pm 0.026$ & $12.357 \pm 0.028$ & $12.479 \pm 0.021$ & $12.445 \pm 0.014$ & $12.747 \pm 0.021$ \\
\hline 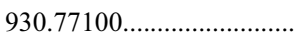 & $11.736 \pm 0.043$ & $12.358 \pm 0.028$ & $12.469 \pm 0.015$ & $12.446 \pm 0.014$ & $12.743 \pm 0.021$ \\
\hline 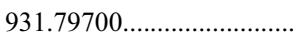 & $11.785 \pm 0.045$ & $12.365 \pm 0.017$ & $12.458 \pm 0.019$ & $12.434 \pm 0.016$ & $12.774 \pm 0.020$ \\
\hline 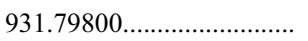 & $11.771 \pm 0.054$ & $12.366 \pm 0.017$ & $12.465 \pm 0.013$ & $12.436 \pm 0.015$ & $12.768 \pm 0.020$ \\
\hline 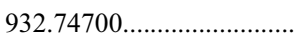 & $11.825 \pm 0.016$ & $12.375 \pm 0.020$ & $12.465 \pm 0.017$ & $12.443 \pm 0.017$ & $12.843 \pm 0.026$ \\
\hline 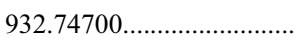 & $11.819 \pm 0.019$ & $12.378 \pm 0.019$ & $12.467 \pm 0.017$ & $12.454 \pm 0.013$ & $12.816 \pm 0.031$ \\
\hline 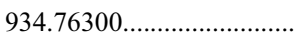 & $11.993 \pm 0.017$ & $12.448 \pm 0.023$ & $12.499 \pm 0.018$ & $12.441 \pm 0.017$ & $12.886 \pm 0.025$ \\
\hline 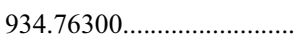 & $11.981 \pm 0.012$ & $12.451 \pm 0.023$ & $12.506 \pm 0.013$ & $12.451 \pm 0.019$ & $12.878 \pm 0.032$ \\
\hline 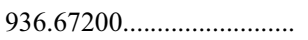 & $12.118 \pm 0.041$ & $12.558 \pm 0.027$ & $12.547 \pm 0.017$ & $12.529 \pm 0.026$ & $12.971 \pm 0.022$ \\
\hline 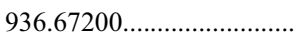 & $12.117 \pm 0.052$ & $12.560 \pm 0.022$ & $12.563 \pm 0.015$ & $12.526 \pm 0.018$ & $12.966 \pm 0.027$ \\
\hline 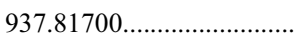 & $12.234 \pm 0.042$ & $12.634 \pm 0.024$ & $12.590 \pm 0.018$ & $12.579 \pm 0.016$ & $13.091 \pm 0.038$ \\
\hline 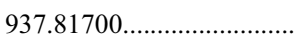 & $12.245 \pm 0.023$ & $12.633 \pm 0.025$ & $12.597 \pm 0.024$ & $12.577 \pm 0.022$ & $13.084 \pm 0.032$ \\
\hline 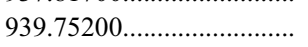 & $12.453 \pm 0.052$ & $12.767 \pm 0.047$ & $12.686 \pm 0.016$ & $12.715 \pm 0.017$ & $13.190 \pm 0.020$ \\
\hline 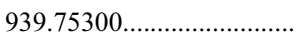 & $12.429 \pm 0.034$ & $12.778 \pm 0.029$ & $12.681 \pm 0.013$ & $12.716 \pm 0.018$ & $13.199 \pm 0.016$ \\
\hline 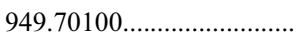 & $13.799 \pm 0.026$ & $13.843 \pm 0.022$ & $13.286 \pm 0.026$ & $13.249 \pm 0.019$ & $13.389 \pm 0.029$ \\
\hline $949.70100 \ldots \ldots \ldots \ldots \ldots \ldots . .$. & $13.812 \pm 0.016$ & $13.837 \pm 0.031$ & $13.312 \pm 0.023$ & $13.303 \pm 0.020$ & $13.400 \pm 0.024$ \\
\hline 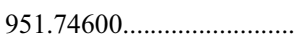 & $14.116 \pm 0.025$ & $14.118 \pm 0.021$ & $13.405 \pm 0.019$ & $13.294 \pm 0.032$ & $13.336 \pm 0.030$ \\
\hline $951.74600 \ldots \ldots \ldots \ldots \ldots \ldots \ldots \ldots$ & $14.118 \pm 0.028$ & $14.126 \pm 0.024$ & $13.417 \pm 0.016$ & $13.281 \pm 0.013$ & $13.337 \pm 0.025$ \\
\hline 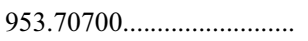 & $14.416 \pm 0.029$ & $14.361 \pm 0.020$ & $13.524 \pm 0.018$ & $13.308 \pm 0.028$ & $13.289 \pm 0.028$ \\
\hline 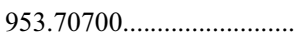 & $14.403 \pm 0.055$ & $14.369 \pm 0.029$ & $13.527 \pm 0.018$ & $13.342 \pm 0.023$ & $13.289 \pm 0.025$ \\
\hline 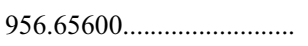 & $14.787 \pm 0.035$ & $14.686 \pm 0.031$ & $13.708 \pm 0.024$ & $13.368 \pm 0.029$ & $13.226 \pm 0.020$ \\
\hline 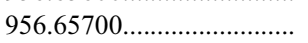 & $14.775 \pm 0.045$ & $14.714 \pm 0.019$ & $13.667 \pm 0.032$ & $13.408 \pm 0.025$ & $13.267 \pm 0.026$ \\
\hline $960.69600 \ldots \ldots \ldots \ldots \ldots \ldots \ldots \ldots$ & $15.130 \pm 0.024$ & $15.009 \pm 0.019$ & $13.892 \pm 0.020$ & $13.514 \pm 0.020$ & $13.231 \pm 0.018$ \\
\hline 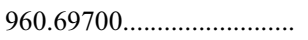 & $15.132 \pm 0.018$ & $15.018 \pm 0.017$ & $13.902 \pm 0.024$ & $13.536 \pm 0.040$ & $13.235 \pm 0.025$ \\
\hline 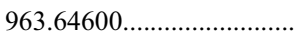 & $15.243 \pm 0.051$ & $15.211 \pm 0.017$ & $14.063 \pm 0.015$ & $13.724 \pm 0.038$ & $13.358 \pm 0.024$ \\
\hline 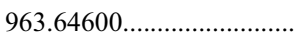 & $15.273 \pm 0.039$ & $15.209 \pm 0.020$ & $14.102 \pm 0.051$ & $13.692 \pm 0.015$ & $13.365 \pm 0.022$ \\
\hline 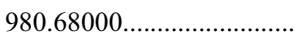 & $15.773 \pm 0.029$ & $15.785 \pm 0.034$ & $14.859 \pm 0.037$ & $14.526 \pm 0.017$ & $14.375 \pm 0.017$ \\
\hline $982.70100 \ldots \ldots \ldots \ldots \ldots \ldots \ldots \ldots . .$. & $15.829 \pm 0.046$ & $15.778 \pm 0.048$ & $14.940 \pm 0.050$ & $14.654 \pm 0.033$ & $14.476 \pm 0.036$ \\
\hline $987.69200 \ldots \ldots \ldots \ldots \ldots \ldots . .$. & $15.877 \pm 0.029$ & $15.828 \pm 0.013$ & $14.965 \pm 0.020$ & $14.756 \pm 0.017$ & $14.713 \pm 0.018$ \\
\hline $987.69300 \ldots \ldots \ldots \ldots \ldots \ldots . .$. & $\ldots$ & $\ldots$ & $14.946 \pm 0.017$ & & $\ldots$ \\
\hline 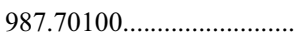 & $\ldots$ & & $14.954 \pm 0.018$ & $\ldots$ & $\ldots$ \\
\hline 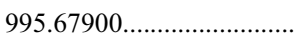 & $16.014 \pm 0.032$ & $15.942 \pm 0.018$ & $15.161 \pm 0.019$ & $14.998 \pm 0.016$ & $15.080 \pm 0.024$ \\
\hline $1010.6530 \ldots \ldots \ldots \ldots \ldots \ldots \ldots \ldots$ & $16.388 \pm 0.035$ & $16.138 \pm 0.015$ & $15.560 \pm 0.012$ & $15.503 \pm 0.011$ & $15.691 \pm 0.026$ \\
\hline $1013.6570 \ldots \ldots \ldots \ldots \ldots \ldots \ldots \ldots$ & $16.470 \pm 0.033$ & $16.197 \pm 0.024$ & $15.642 \pm 0.023$ & $15.593 \pm 0.019$ & $15.806 \pm 0.023$ \\
\hline $1013.6630 \ldots \ldots \ldots \ldots \ldots \ldots \ldots \ldots$ & $\ldots$ & $\ldots$ & $15.650 \pm 0.018$ & $\ldots$ & $\ldots$ \\
\hline 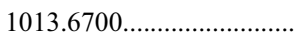 & $\ldots$ & & $15.639 \pm 0.020$ & $\ldots$ & $\ldots$ \\
\hline 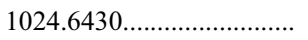 & $16.648 \pm 0.043$ & $16.346 \pm 0.032$ & $15.913 \pm 0.019$ & $15.941 \pm 0.027$ & $16.214 \pm 0.031$ \\
\hline $1137.0280 \ldots \ldots \ldots \ldots \ldots . .$. & $19.328 \pm 0.142$ & $18.090 \pm 0.019$ & $18.028 \pm 0.077$ & $18.625 \pm 0.145$ & $18.587 \pm 0.044$ \\
\hline $1195.9130 \ldots \ldots \ldots \ldots \ldots \ldots . .$. & $20.455 \pm 0.336$ & $18.956 \pm 0.031$ & $18.833 \pm 0.087$ & $19.599 \pm 0.144$ & $\ldots$ \\
\hline $1216.9110 \ldots \ldots \ldots \ldots \ldots \ldots \ldots . .$. & $\ldots$ & $19.299 \pm 0.032$ & $19.291 \pm 0.035$ & & \\
\hline 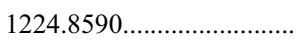 & $\ldots$ & $19.433 \pm 0.067$ & $19.427 \pm 0.039$ & $20.146 \pm 0.230$ & $19.998 \pm 0.167$ \\
\hline $1275.8010 \ldots \ldots \ldots \ldots \ldots \ldots \ldots \ldots$ & $\ldots$ & $20.013 \pm 0.074$ & $20.140 \pm 0.131$ & & $\ldots$ \\
\hline
\end{tabular}

Note.-Table 12 is also available in machine-readable form in the electronic edition of the Astrophysical Journal.

${ }^{\mathrm{a}}$ MJD is the Julian Date minus 2,450,000. 

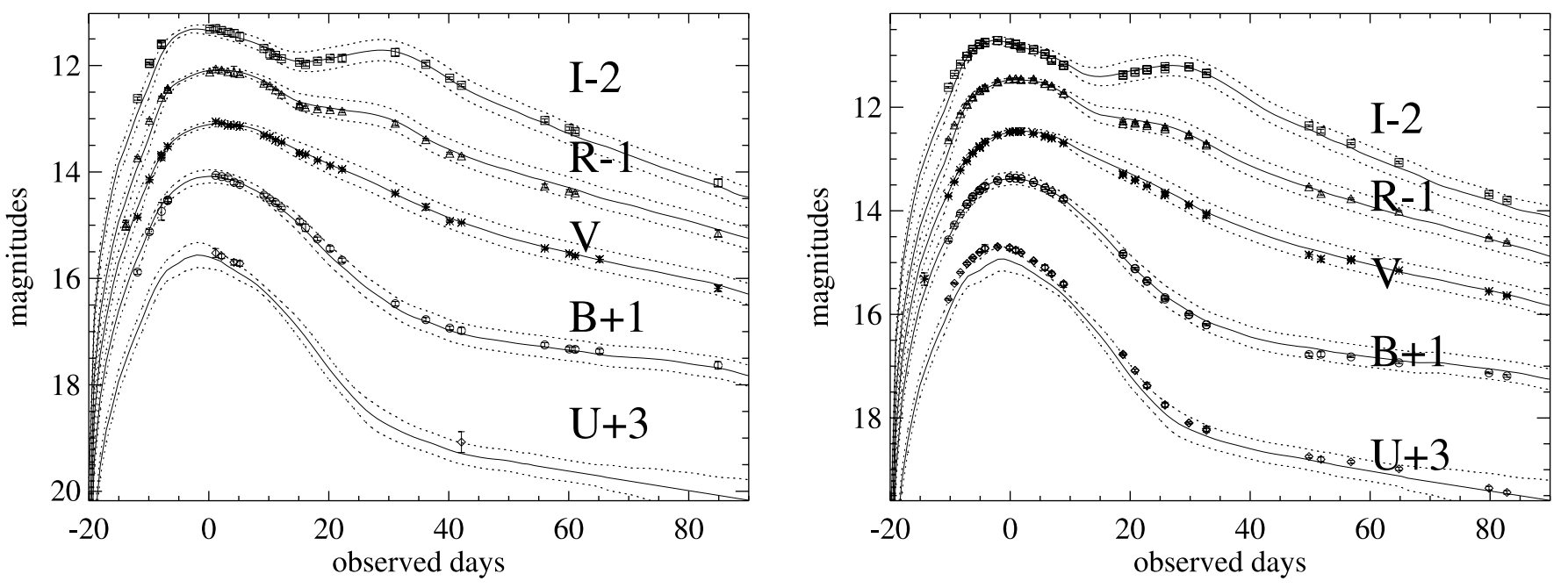

FIG. 12.-MLCS2k2 fits to the UBVRI-band data for SN 1994ae and SN 1998aq.

set, the only free parameters for an individual SN Ia are $t_{0}, \mu_{0}$, $\Delta$, and $A_{V}^{0}$.

To determine the unreddened colors of the fiducial SN Ia, MLCS2k2 uses the observation that by $\sim 35$ days after $B$ maximum, when SNe Ia have entered the nebular phase, their colors are uniform (empirically shown by Lira 1996; Riess et al. 1996a). Any variation from this color is assumed to be due to reddening and intrinsic dispersion. Thus, the distribution of observed nebular colors can be fitted with three parameters: a fiducial color, the intrinsic dispersion of the Gaussian distribution of colors, and the scale of an exponentially decaying distribution of reddenings (motivated by simulations of host opacities from Hatano et al. 1998). Using the derived, unreddened nebular colors, all SNe Ia are dereddened and utilized to derive the $\boldsymbol{P}_{X}$ and $\boldsymbol{Q}_{X}$ vectors. The end result is a determination that the fiducial SN Ia [which for MLCS2k2 has $\Delta m_{15}(B)=1.07 \mathrm{mag}$ ] has unreddened colors at $B$-band maximum of $U-B=-0.40, B-V=-0.11$, $V-R=0.00$, and $V-I=-0.30 \mathrm{mag}$.

For an individual SN Ia, the time-dependent luminosity is described by $\boldsymbol{M}_{X}^{0}+\boldsymbol{P}_{X} \Delta+\boldsymbol{Q}_{X} \Delta^{2}$, where the sum of the second and third terms represents the difference or correction to the luminosity from the fiducial to the individual SN Ia. The zero point or distance scale is set by a determination of $\boldsymbol{M}_{X}^{0}$ at any phase (e.g., at peak).

Here we measure the fiducial luminosity by using the Cepheid calibrations of the distances to the hosts of SN 1994ae and SN 1998aq (now treated as the true distances) and evaluating

$$
\boldsymbol{M}_{X}^{0}=\boldsymbol{m}_{X}\left(t-t_{0}\right)-\mu_{\text {Cepheid }, 0}-\boldsymbol{\eta}_{X} A_{V}^{0}-\boldsymbol{P}_{X} \Delta-\boldsymbol{Q}_{X} \Delta^{2},
$$

where $\mu_{\text {Cepheid }, 0}$ is the Cepheid-calibrated distance from the previous section.
The MLCS2k2 parameters derived from the fits are given in Table 13. The parameter of greatest interest, $M_{V}^{0}\left(t_{0}\right)$, is $-19.18 \pm$ 0.13 and $-19.19 \pm 0.13$ mag for the new objects SN 1994ae and SN 1998aq, respectively, where the uncertainty listed contains only the uncorrelated (random) uncertainty as discussed in the next section. A simple average of the four ideal calibrators yields $M_{V}^{0}\left(t_{0}\right)=-19.17 \pm 0.07 \mathrm{mag}$ (the more accurate weighted average computed in the next section yields the same result).

Interestingly, the two "corrections" to the brightness of SN 1994ae and SN 1998aq, $\Delta$ and $A_{V}^{0}$, are nearly identical and offset each other (e.g., $\Delta+A_{V}^{0} \approx 0 \mathrm{mag}$ ), indicating that in the absence of any corrections for intrinsic luminosity and extinction, the estimated distance moduli would remain virtually identical.

We have utilized the "Gold" sample of SNe Ia from Riess et al. (2004) to measure the global Hubble flow of SNe Ia. This full list is a complete, "high-confidence" set of 157 objects that includes all SNe Ia not suffering from one or more known sources of systematic uncertainty. Reasons for exclusion from the Gold sample are (1) an uncompelling classification or (2) an incomplete or potentially inaccurate photometric record (including limited light-curve sampling, low-precision color information, or non-CCD measurements). An additional reason for exclusion was a large measured extinction $\left(A_{V}>1 \mathrm{mag}\right)$, amplifying systematic errors from potentially non-Galactic reddening laws.

The relative distances of this sample were previously used to characterize mass-energy components of the universe. However, the zero point or distance scale was set to $M_{V}^{0}\left(t_{0}\right)=-19.44 \mathrm{mag}$ in accordance with Cepheid analyses by the STS group. The values of $M_{V}^{0}\left(t_{0}\right)$ provided in Table 12 allow us to set the luminosity of the SNe Ia in the Gold sample to the Cepheid calibrations

TABLE 13

MLCS2k2 PARAMETERS

\begin{tabular}{|c|c|c|c|c|c|c|}
\hline $\mathrm{SN}$ & $\begin{array}{l}\mu_{\text {Cepheid, } 0}{ }^{\mathrm{a}} \\
\quad \text { (mag) }\end{array}$ & $\begin{array}{c}M_{V}^{0}\left(t_{0}\right) \\
(\mathrm{mag})\end{array}$ & $\begin{array}{c}\Delta \\
(\mathrm{mag})\end{array}$ & $\begin{array}{c}A_{V}^{0} \\
(\mathrm{mag})\end{array}$ & $t_{0}{ }^{\mathrm{b}}$ & $\begin{array}{c}H_{0} \\
\left(\mathrm{~km} \mathrm{~s}^{-1} \mathrm{Mpc}^{-1}\right)\end{array}$ \\
\hline 1994ae .............. & $32.29 \pm 0.06$ & $-19.18 \pm 0.12$ & $-0.33 \pm 0.03$ & $0.30 \pm 0.03$ & $9684.84 \pm 0.35$ & 72 \\
\hline $1998 \mathrm{aq} \ldots \ldots \ldots \ldots . . . . .$. & $31.66 \pm 0.09$ & $-19.19 \pm 0.12$ & $-0.05 \pm 0.02$ & $0.05 \pm 0.03$ & $50930.85 \pm 0.10$ & 73 \\
\hline $1990 \mathrm{~N} \ldots \ldots \ldots \ldots$ & $31.74 \pm 0.12$ & $-19.10 \pm 0.12$ & $-0.32 \pm 0.06$ & $0.42 \pm 0.05$ & $8081.90 \pm 0.14$ & 75 \\
\hline 1981B ................ & $30.86 \pm 0.09$ & $-19.21 \pm 0.12$ & $-0.19 \pm 0.03$ & $0.52 \pm 0.05$ & $44670.71 \pm 0.88$ & 72 \\
\hline
\end{tabular}

a Statistical uncertainty from dereddened $P-L$ fit and $[\mathrm{O} / \mathrm{H}]$ measurement only.

b The Julian Date minus 2,400,000. 
TABLE 14

Error Budget for $H_{0}$ in Units of Magnitudes

\begin{tabular}{|c|c|c|c|c|}
\hline Source & 1994ae (ACS) & 1998aq (WFPC2) & 1990N (WFPC2) & 1981B (WFPC2) \\
\hline \multicolumn{5}{|c|}{ Random Error Sources } \\
\hline 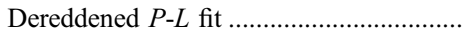 & 0.05 & 0.08 & 0.09 & 0.07 \\
\hline 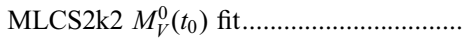 & 0.12 & 0.12 & 0.12 & 0.12 \\
\hline 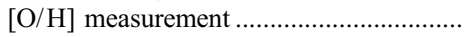 & 0.03 & 0.03 & 0.08 & 0.05 \\
\hline 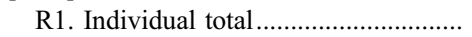 & 0.133 & 0.147 & 0.170 & 0.147 \\
\hline 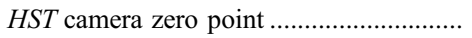 & $0.03(\mathrm{ACS})$ & & $\longleftarrow 0.03(\mathrm{WFPC} 2) \longrightarrow$ & \\
\hline R2. Effect on dereddened $P-L \ldots \ldots \ldots \ldots$ & $0.086(\mathrm{ACS})$ & & $\longleftarrow 0.086(\mathrm{WFPC} 2) \longrightarrow$ & \\
\hline $\mathrm{R} 12=\mathrm{R} 1+\mathrm{R} 2$ (ACS/WFPC2) $\ldots \ldots \ldots \ldots \ldots$ & $0.158(\mathrm{ACS})$ & & $\longleftarrow 0.124(\mathrm{WFPC} 2) \longrightarrow$ & \\
\hline 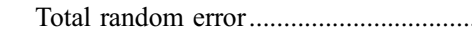 & ..................... & & 0.098 & \\
\hline
\end{tabular}

Systematic Error Sources

\begin{tabular}{|c|c|}
\hline 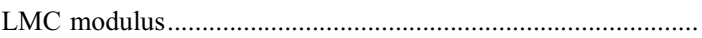 & 0.10 \\
\hline 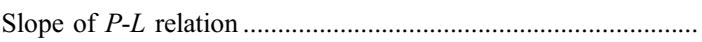 & 0.05 \\
\hline 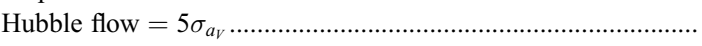 & 0.025 \\
\hline 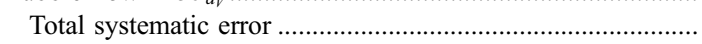 & 0.115 \\
\hline
\end{tabular}

Total Error

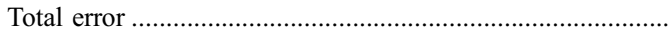

0.150

presented here, resulting in a constant decrease of all distances in Riess et al. (2004) by $M_{V}^{0}\left(t_{0}\right)-19.44=0.27 \mathrm{mag}$.

The redshift range used to measure the global expansion rate has a nontrivial impact on the inferred value. Zehavi et al. (1998) first noted a possible kinematic ripple in the Hubble diagram of SNe Ia at $c z=7000 \mathrm{~km} \mathrm{~s}^{-1}$, representing a few percent change in the Hubble constant across this feature. To measure the true, global expansion rate free of undue influence by flows and infalls, we have set the boundary beyond this feature. To reduce any "cosmological bias" depending on the absence or presence of a $70 \%$ fraction of vacuum energy, we have set an upper limit of $z=0.08$ to include the greatest number of SNe Ia while limiting such bias to less than $2 \%$ of the Hubble constant at an average redshift $z=0.04$. The $38 \mathrm{SNe}$ Ia from the Gold sample in this redshift range are SN 1998eg, SN 1994M, SN 1993H, SN 1999X, SN 1999gp, SN 1992ag, SN 1992P, SN 2000bk, SN 1996C, SN 1993ah, SN 1994Q, SN 1997dg, SN 19900, SN 1999cc, SN 1998cs, SN 1991U, SN 1996bl, SN $1994 \mathrm{~T}, \mathrm{SN} 1992 \mathrm{bg}, \mathrm{SN} 2000 \mathrm{cf}, \mathrm{SN} 1999 \mathrm{ef}, \mathrm{SN} 1990 \mathrm{~T}, \mathrm{SN}$ 1992bl, SN 1992bh, SN 1992J, SN 1995ac, SN 1993ac, SN 1990af, SN 1993ag, SN 1993O, SN 1998dx, SN 1991S, SN 1992bk, SN 1992au, SN 1992bs, SN 1993B, SN 1992ae, and SN 1992bp.

Following Jha et al. (1999), it is useful to define a distance scale-independent intercept of the Hubble flow SNe Ia, $a_{V}$, where $a_{V} \equiv \log (c z)-0.2 m_{V}^{0}\left(t_{0}\right)=\log H_{0}-0.2 M_{V}^{0}\left(t_{0}\right)-5$, which we find to be $0.697 \pm 0.005$ in units of 0.2 mag for the Gold sample of Riess et al. (2004) at $0.023<z<0.080 .{ }^{10}$ The value of $H_{0}$ for each ideal calibrator is then given in Table 13 for its individual value of $M_{V}^{0}\left(t_{0}\right)$.

\subsection{Error Budget}

To calculate the weighted average value of $H_{0}$ for all ideal calibrators and its uncertainty, it is necessary to distinguish between errors that are correlated or uncorrelated among the individual cal-

\footnotetext{
${ }^{10}$ The specific value of $a_{V}$, although independent of distance scale, does depend on the arbitrary choice of a fiducial SN Ia, but this dependence is removed from the determination of $H_{0}$.
}

ibrators and thus may or may not be reduced by averaging. The propagation of uncertainty is tabulated in Table 14 and described here.

Uncorrelated, random errors are dominated by the dispersion of ideal calibrators. Although the statistical uncertainty of such well-sampled light curves as these can be $<0.05$ mag, the dispersion appears to be dominated by an intrinsic component (Riess et al. 1996a; Perlmutter et al. 1997; Phillips et al. 1999; Tripp \& Branch 1999; S. Jha et al. 2005, in preparation). Estimates of this intrinsic component range from 0.10 to $0.17 \mathrm{mag}$. Due to the highly overdetermined calibration and light-curve sampling of these objects, we take this dispersion per object to be 0.12 mag. Additional random errors result from the uncertainty of the dereddened $P-L$ fit (intercept) and the measurement of the host metallicities. (Errors due to differences in the host extinction laws from the standard Galactic reddening law are negligible because the reddenings are small and such errors are mostly removed by dereddening both the $\mathrm{SNe}$ and the Cepheids.) These random error sources can be added in quadrature and are assumed to be independent for each individual collaborator.

Each of the two HST cameras, WFPC2 and ACS, is assumed to have independent 0.03 mag uncertainties in their zero points in both the $V$ and $I$ bands. After dereddening the Cepheids, the resultant uncertainty is $0.086 \mathrm{mag}$ (i.e., $\left[\sigma_{V}^{2}(1-R)^{2}+\sigma_{I}^{2} R^{2}\right]^{1 / 2}$ ) for each camera. For the three calibrations obtained with WFPC2, the $0.086 \mathrm{mag}$ error is correlated but is assumed to be uncorrelated with the 0.086 mag for the single ACS calibration. We sum in quadrature the camera-dependent error with the above individual errors separately for the SNe calibrated with ACS and WFPC2.

The weighted average value of $H_{0}$ for the four calibrators is computed in proportion to their uncorrelated error components (i.e., the individual errors determine the weights within the subtotal for each camera and then are combined with the independent camera errors to reach the total as shown in Table 14).

Correlated errors affecting all calibrators include an assumed 0.10 mag uncertainty for the distance to the LMC (i.e., the consensus value of the STS and SKP groups), a 0.05 mag uncertainty for the form (i.e., slope) of the $P-L$ relation, and the $0.025 \mathrm{mag}$ uncertainty determined by the fit to the ridgeline of the $37 \mathrm{SNe}$ Ia 
TABLE 15

SN ObSERVABles

\begin{tabular}{ccccccc}
\hline \hline SN & $\begin{array}{c}U_{\max } \\
(\mathrm{mag})\end{array}$ & $\begin{array}{c}B_{\max } \\
(\mathrm{mag})\end{array}$ & $\begin{array}{c}V_{\max } \\
(\mathrm{mag})\end{array}$ & $\begin{array}{c}R_{\max } \\
(\mathrm{mag})\end{array}$ & $\begin{array}{c}I_{\max } \\
(\mathrm{mag})\end{array}$ & $\begin{array}{c}\Delta m_{15} \\
(B)\end{array}$ \\
\hline SN 1994ae.......... & $12.45 \pm 0.04$ & $13.08 \pm 0.02$ & $13.08 \pm 0.02$ & $13.07 \pm 0.03$ & $13.29 \pm 0.04$ & $0.90 \pm 0.03$ \\
SN 1998aq ........ & $11.70 \pm 0.03$ & $12.36 \pm 0.02$ & $12.46 \pm 0.02$ & $12.43 \pm 0.02$ & $12.72 \pm 0.02$ & $1.05 \pm 0.03$ \\
\hline
\end{tabular}

defining the Hubble flow from the Gold sample. The $25 \%$ systematic uncertainty in the metallicity correction from Sakai et al. (2004) is negligible compared to the other systematic error sources. Together, these errors provide an irreducible, systematic uncertainty of 0.115 mag.

The combined uncertainty for the four measurements is $1 \sigma=0.10 \mathrm{mag}$ for the random component (now including the zero points of the $H S T$ cameras) and $1 \sigma=0.115 \mathrm{mag}$ for the systematic component, for a total of $0.15 \mathrm{mag}$, or $8 \%$ in the value of $H_{0}$. Weighting the average of the four calibrators (in Table 13) in proportion to the uncorrelated error components yields

$$
\begin{aligned}
H_{0} & =73 \pm 4 \text { (statistical) } \pm 5 \text { (systematic) } \\
& =73 \pm 6.4 \text { (total) } \mathrm{km} \mathrm{s}^{-1} \mathrm{Mpc}^{-1} .
\end{aligned}
$$

To test the sensitivity of our results to the adopted light-curve fitting method, here we also applied the $\Delta m_{15}(B)$ fitting method as calibrated by Suntzeff et al. (1999) and Phillips et al. (1999) and using the fitted peak apparent magnitudes and $\Delta m_{15}(B)$ parameters given in Table $15 .{ }^{11}$ The result was a value of $H_{0}=71 \mathrm{~km} \mathrm{~s}^{-1}$ $\mathrm{Mpc}^{-1}$ (with a similar error budget as the one in Table 14), in good agreement with the MLCS2k2 method. ${ }^{12}$ We have also attempted to reproduce the methodology of the STS group and by their methods find an average value of $H_{0}=69 \mathrm{~km} \mathrm{~s}^{-1} \mathrm{Mpc}^{-1}$ for SN 1994ae and SN 1998aq. ${ }^{13}$ Following the SKP methodology yields

11 For each of the $U B V R I$-band light curves, we fitted a closely matching lightcurve segment to the data in a small range near maximum brightness $(-10$ days $<$ $t<15$ days). The best-fit peak apparent magnitudes are then given in Table 15 . The high rate of data sampling allowed us to estimate the values of $\Delta m_{15}(B)$ directly as given in Table 15. Following Phillips et al. (1999), the observed value of $\Delta m_{15}(B)$ for SN 1994 ae should be reduced by $0.01 \mathrm{mag}$ (to $0.89 \mathrm{mag}$ ) to account for the light-curve narrowing effect of $E(B-V) \approx 0.1 \mathrm{mag}$.

12 From Phillips et al. (1999), we use eqs. (7) and (8), which express the unreddened pseudocolor $B_{\max }-V_{\max }$ and $V_{\max }-I_{\max }$ of an SN Ia; we find $E(B-V)=0.11 \pm 0.04 \mathrm{mag}$ and $E(V-I)=0.17 \pm 0.04 \mathrm{mag}$ for SN 1994ae, and $E(B-V)=-0.02 \pm 0.04 \mathrm{mag}$ and $E(V-I)=0.07 \pm 0.04 \mathrm{mag}$ for SN 1998aq. Using a standard extinction law (Cardelli et al. 1989), the average extinction for SN 1994ae and SN 1998aq is $A_{V}=0.26 \pm 0.10$ and $0.04 \pm 0.10 \mathrm{mag}$, respectively. Using the expression from Suntzeff et al. (1999), $\log H_{0}(V)=$ $0.2\left\{M_{\max }^{V}-0.672\left[\Delta m_{15}(B)-1.1\right]-0.633\left[\Delta m_{15}(B)-1.1\right]^{2}+28.590\right\}$, yields $H_{0}=70$ and $76 \mathrm{~km} \mathrm{~s}^{-1} \mathrm{Mpc}^{-1}$, respectively. For SN 1990N and SN 1981B using the parameters from Suntzeff et al. (1999) and our OGLE+10 $P$ - $L$ relationbased estimate of the Cepheid distances to the hosts yields $H_{0}=69$ and $70 \mathrm{~km} \mathrm{~s}^{-1}$ $\mathrm{Mpc}^{-1}$, respectively. The average value of the four ideal calibrators is thus $71 \mathrm{~km} \mathrm{~s}^{-1} \mathrm{Mpc}^{-1}$.

${ }^{13}$ We can try to estimate the values of $H_{0}$ that the STS collaboration would find from these two new calibrators (of course, we cannot be sure what they will find until they analyze the data presented here themselves). To reproduce the STS prescription, we take the $V$ at peak of 12.46 mag from Table 15 and subtract the Saha et al. (2001) Cepheid value of $\mu_{0}=31.72 \mathrm{mag}$ to yield $M_{V}^{0}\left(t_{0}\right)=$ -19.26 mag. Assuming that STS would find no significant corrections resulting from the low apparent reddening and typical light-curve shape seen for SN 1998aq, we find that $M_{V}^{0}\left(t_{0}\right)$ is fainter than that in Parodi et al. (2000) by 0.27 mag, yielding $H_{0}$ that is $13.5 \%$ larger than that of Parodi et al. (2000), or $H_{0}=69 \mathrm{~km} \mathrm{~s}^{-1} \mathrm{Mpc}^{-1}$. The difference between this and the value $H_{0}=60 \mathrm{~km} \mathrm{~s}^{-1} \mathrm{Mpc}^{-1}$ from STS for SN 1998aq results from the misestimate of the peak $V$ used by Saha et al. (2001), which was based on a rough, amateur observation at a single phase and apparently 0.18 mag too bright as compared with the complete photometric record presented here. For SN 1994ae, using the STS method value of $\mu_{w}=32.36 \mathrm{mag}$ (see $\S 2.3$ ) and the similar lack of significant corrections results in $M_{V}^{0}=13.08-$ $32.36=-19.28 \mathrm{mag}$ for a similar result of $H_{0}=68 \mathrm{~km} \mathrm{~s}^{-1} \mathrm{Mpc}^{-1}$. an average value of $H_{0}=75 \mathrm{~km} \mathrm{~s}^{-1} \mathrm{Mpc}^{-1}$. Although we conclude that the inferred value of $H_{0}$ from this set of SN Ia calibrators is not sensitive to the light-curve shape method, we invite the application of other fitting prescriptions to the data presented here. The striking reduction in the difference between the value of $H_{0}$ estimated by the prescriptions of different groups results directly from the improvements in data quality as enumerated in the next section.

\section{DISCUSSION}

The "era of precision cosmology" has been realized as an unprecedented time when it has become possible to constrain each of the cosmological parameters that characterize our universe to an uncertainty of less than $10 \%$. Arguably the most fundamental of the parameters and the one to which the greatest effort has been applied is the Hubble constant. Unfortunately, its estimation has remained highly controversial, suffering from direct propagation of errors along a long chain of "nuisance parameters" (e.g., the distance to the LMC). SNe Ia are the most precise, long-range distance indicator known, and their accurate calibration lies at the heart of estimating $H_{0}$. The two most active groups employing SNe Ia to estimate $H_{0}$, the Key Project and the STS collaboration, disagree by $20 \%$ in their latest (perhaps final?) attempts. We believe that the resolution of this difference is a prerequisite to adding $H_{0}$ to the ledger of the well-calibrated parameters.

In Table 16 we summarize the primary sources of differences between these two groups and our own analysis that lead to the $20 \%$ discrepancy in the value of $H_{0}$.

We conclude that much of the discrepancy is caused by the challenges of wringing measurements from low-quality or

TABLE 16

\begin{tabular}{|c|c|c|}
\hline Source & $\begin{array}{c}\Delta H_{0} \text { STS versus } \\
\text { This Paper }\end{array}$ & $\begin{array}{c}\Delta H_{0} \mathrm{STS} \\
\text { versus SKP }\end{array}$ \\
\hline Use of nonideal calibrators ${ }^{\mathrm{a}}$ & $\downarrow 7 \%$ & $\downarrow 1 \%$ \\
\hline Form of $P-L$ relation................... & $\downarrow 5 \%$ & $\downarrow 7 \%$ \\
\hline Measured extinction ${ }^{\mathrm{b}}$.................... & $\downarrow 5 \%$ & $\downarrow 5 \%$ \\
\hline Lack of metallicity correction ..................... & $\uparrow 4 \%$ & $\uparrow 4 \%$ \\
\hline WFPC2 "long versus short effect" ........... & $\downarrow 2.5 \%$ & $\downarrow 2.5 \%$ \\
\hline 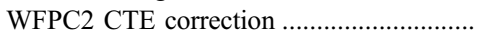 & $\downarrow 2 \%$ & $\downarrow 2 \%$ \\
\hline 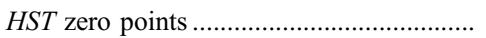 & $\downarrow 1.5 \%$ & $\downarrow 3 \%$ \\
\hline Use of pre-1980 SNe Ia, Hubble flow ${ }^{\mathrm{c}} \ldots . .$. & $\downarrow 1 \%$ & $\downarrow 1 \%$ \\
\hline Strength of luminosity correction ................ & $\downarrow 1 \%$ & $\downarrow 1 \%$ \\
\hline 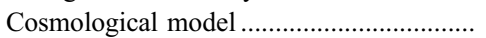 & $\downarrow 1 \%$ & $\downarrow 1 \%$ \\
\hline 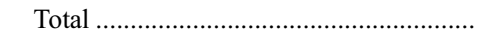 & $\downarrow 22 \%$ & $\downarrow 20 \%$ \\
\hline
\end{tabular}

Sources of Differences in Estimation of $H_{0}$ using SNe Ia

${ }^{\text {a }}$ Differences in $M_{V}^{0}$ from Saha et al. (2001) for SN 1981B and SN 1990N vs. the rest.

${ }^{b}$ STS use a redder intrinsic color resulting in less extinction, most affecting SNe Ia in Cepheid hosts; estimated from the difference in $A_{V}$ for SN 1981B + SN $1990 \mathrm{~N}$ and the Gold sample.

${ }^{c}$ Pre-1980 vs. post-1980 SN Ia average $M_{V}^{0}$ from Parodi et al. (2000) is 0.09 mag. Pre- $1980 \mathrm{SNe}$ Ia are $25 \%$ of sample. 
problematic data: photographic photometry, highly extinguished or extreme SNe Ia, poorly sampled light curves, and cameras with photometric anomalies such as the "long versus short effect" (Holtzman et al. 1995) and charge transfer efficiency (CTE; e.g., Whitmore et al. 1999). We estimate that together these effects account for fully $\sim 17 \%$ of the $22 \%$ difference in $H_{0}$ measurements in this paper and a similar fraction for the SKP (see Table 16). Debatable differences like the strength of the light-curve shapeluminosity relation impact $H_{0}$ by only $1 \%$ (for comparison see Saha et al. 2001). The most expedient way to overcome the challenges of poor data is to supercede the analysis with higher quality data. Using the ideal calibrators presented here, we conclude that the remaining differences in the two teams' approaches would appear to affect the Hubble constant by only $\sim 5 \%{ }^{14}$

\subsection{More Accurate $H_{0}$ through Better SN Ia Data}

The renaissance in the use of $\mathrm{SNe}$ Ia for precision cosmology has been fueled by highly precise and accurate photometry. Large samples ( $>10$ objects) of CCD photometry, frequently using galaxy subtraction techniques and consistent Landolt (1992) standards, have been published by the Calán/Tololo Survey (Hamuy et al. 1996b), the CfA Survey I (Riess et al. 1999a), and the CfA Survey II (Jha et al. 2005). These data sets have individual photometric measurements with absolute zero points known to better than 5\% and lead to Hubble diagrams with dispersions of less than $0.2 \mathrm{mag}$.

Photographic observations obtained at the Asiago Observatory (and elsewhere) of SNe Ia in the 1950s through the early 1980 s, while valuable for elucidating the general properties of $\mathrm{SNe}$ Ia, suffer from a significant lack of accuracy. Despite recent efforts to recalibrate their standard-star sequences digitally (Patat et al. 1997), these light curves still suffer from the original inaccuracy in their SN magnitude estimates. As described by Patat et al. (1997), "SN magnitudes, especially for objects lying on the luminous background of their parent galaxies, were often obtained by eye comparison," a statement that still describes the current state of photometry for these supernovae even after adjustments to the comparison stars. Patat et al. (1997) estimate that the errors from the by-eye estimates are typically $\sim 0.1$ mag (or 3-4 times this value after reddening correction), with noted discrepancies of up to 0.6 mag from expected behavior at late times due to "the poor contrast of the supernova against the parent galaxy background." The mean $B-V$ at peak of the sample has changed by $0.06 \mathrm{mag}$ (again, 3-4 times this for reddening-free distance estimates) due only to corrections to the comparison stars. A similar sized, systematic error in the mean may still plague the SN magnitudes based on the original by-eye estimations from the photographic plates. Additional errors may result from the inability to apply "S-corrections" (Suntzeff et al. 1999) to correct for the disparity between Johnson/Cousins passbands and photographic emulsion sensitivities. ${ }^{15}$

Although it is regrettable to discard data and further deplete the already sparse, published sample of nearby $\mathrm{SNe}$ Ia, it is difficult if not improbable to extract accurate estimates of distances, free of $\sim 5 \%-10 \%$ systematic errors, from such data. Despite he-

\footnotetext{
14 Most of this difference arises from the STS collaboration's use of the $P-L$ relation from the MF91 Cepheids, but the superior sampling of the OGLE Cepheids (Udalski et al. 1999) and photometric testing by Sebo et al. (2002) now argue in favor of an OGLE-based $P-L$ relation, closer to what has been adopted by SKP or by Thim et al. (2003).

15 Anecdotally, we note that one photographic observation in the $B$ band of SN 1994ae by Tsvetkov \& Pavlyuk (1997) was obtained contemporaneously with our observations on 1994 December 9, for which Tsvetkov \& Pavlyuk (1997) report the SN to be 0.25 mag brighter than our own CCD measurement.
}

roic efforts to recalibrate old photographically observed SNe Ia whose hosts are near enough to observe Cepheids with WFPC2 (e.g., Schaefer 1994, 1995, 1996), in the interest of accuracy and resolving the debate over the value of $H_{0}$ we discard (and advocate others to discard) the use of SN 1895B, SN 1937C, SN 1960F, and SN 1974G. Moreover, we think that it is unwise to use such objects to help define the Hubble flow or the luminosity versus light-curve shape relation as done by the STS collaboration (Parodi et al. 2000) and others (e.g., Rowan-Robinson 2002). The photometry of SN 1972E, obtained with a photoelectric photometer by Kirshner et al. (1973), is likely better than photographic photometry but still was not obtained in the same way as for the SNe Ia that now define the Hubble flow. As noted in Table 16, the use of pre-1980 SN Ia data (both in the Hubble flow and among calibrators) alone accounts for $8 \%$ of the change in $H_{0}$ from the STS group.

An additional challenge is posed by $\mathrm{SNe}$ Ia with high reddening [e.g., $E(B-V)>0.3 \mathrm{mag}$ ]. Although the color excess can be measured with similar precision as it is for $\mathrm{SNe}$ Ia having low reddening, inaccuracy may arise in applying an estimate of the total-to-differential absorption ratio $\left[R_{V}=A_{V} / E(B-V)\right]$ for the SN host. Galactic-type values are usually assumed, and tests show that these are accurate to $\sim 25 \%$ in the mean. (Likewise, empirical estimation, such as by Tripp \& Branch [1999] and Parodi et al. [2000], of the magnitude-color dependence is applicable in the mean.] However, a $25 \%$ uncertainty in $R_{V}$ will produce an error of $0.2-0.3 \mathrm{mag}$ in the distance modulus for $E(B-V) \approx 0.3$. Large observed extinction may also arise from nonequilibrium processes (e.g., stellar mass loss) and be characterized by unexpected values of $R_{V}$. Riess et al. (2004) and Tonry et al. (2003) relegated SNe Ia with large measured reddening to a "low-confidence" sample. SN 1989B and SN 1998bu each suffer from large $[E(B-V)>0.3 \mathrm{mag}]$ reddening and therefore are not ideal calibrators.

Differences in reddening correction methods may also lead to dispute in the value of $H_{0}$ if the average reddening of Hubble flow SNe Ia diverges from that of calibrators. In fact, this circumstance is likely because calibrators must be chosen from hosts with ongoing massive-star formation (i.e., Cepheids) and its accompanying dust. (This circumstance even occurs to some degree for SN $1990 \mathrm{~N}$ and SN 1981B, where the average $A_{V}$ for these two calibrators exceeds that of SN 1994ae and SN 1998aq by 0.2 mag.)

Also problematic for resolution of the $H_{0}$ debate are $\mathrm{SNe}$ Ia whose light-curve shapes or spectroscopic properties lie far from the mean or median of SNe Ia. The relatively large distance correction implied by the extreme nature of these $\mathrm{SNe}$ amplifies differences in the calibration of the correction techniques. (To those who derive a relatively "weak" correction, others appear to overcorrect these objects, and vice versa.) The second problem is that the relatively low frequency of extreme $\mathrm{SNe}$ Ia, especially in the Hubble flow, reduces the objects available to define the calibration of the correction techniques at the extreme. Because our goal in this study is to resolve the disagreement over the value of $H_{0}$ determined from $\mathrm{SNe}$ Ia, we avoid the use of such extreme or peculiar SNe Ia (and their added controversy) as SN 1991 T and SN 1999by.

What remains are the "ideal" calibrators, objects having typical light-curve shape, exquisite photometry, well-sampled light curves, and low reddening. This set includes SN 1990N, SN 1981B, SN 1994ae, and SN 1998aq. The accuracy and precision of these objects offer the means to reduce the long-standing disagreement over the Hubble constant determined from $\mathrm{SNe}$ Ia. We encourage the reanalysis of these objects and the similarly 
high-confidence SNe Ia in the Hubble flow by others to examine the size of the remaining differences.

\subsection{WFPC2 versus $A C S$}

In the HST Key Project's final report (F01), the uncertainty in the photometric calibration of WFPC 2 is listed as the second largest remaining source of error (after the distance to the LMC), contributing a systematic error of 0.07-0.09 mag (Gibson et al. 2000; F01). Cepheid calibrations with ACS on HST offer several advantages for reducing this source of uncertainty.

The estimated dimming of Cepheids observed by WFPC2 due to imperfect CTE reached 0.05 mag after 8 yr of radiation damage in orbit by the time NGC 3982 was observed (Whitmore et al. 1999; Dolphin 2000). However, significant disagreement exists in the application of CTE corrections, with the STS collaboration applying none to their DoPhot photometry (Saha et al. 2001) and Stetson et al. (1998) utilizing only a time-independent component in their CTE corrections. In contrast, ACS was a new, undamaged camera at the time of our observations with greater initial CTE than WFPC2. Calibrations by Riess \& Mack (2004) indicate that the typical corrections to Cepheid photometry of NGC 3370, obtained 1 yr after ACS installation, are no more than a few millimagnitudes, small enough to be insignificant and, more importantly, uncontroversial.

In addition, there has been disagreement over the existence of a 0.05 mag photometric anomaly on WFPC 2 known as the "long versus short effect." The nature of the effect, first posited by Holtzman et al. (1995), is a net decrease in the flux of short exposures relative to long exposures by $5 \%$, although more recent analysis by Whitmore et al. (1999) indicates that it may result from inclusion of nearby stars in the annuli used to quantify local background levels in dense calibration clusters. Generally, the STS collaboration has explicitly accounted for this effect while the SKP has not. For ACS photometry, no such anomaly is known to exist (indeed, the linearity of the ACS detectors has been shown to exquisite precision and accuracy; Gilliland et al. 2004).

Two more advantages of ACS over WFPC2 are its field of view and pixel sampling, both a factor of 2 greater for ACS. The larger field of view for ACS approximately doubles the number of Cepheids found in host galaxies of the typical angular size encountered in past work. The greater sampling (ACS samples the PSF near the Nyquist limit; WFPC2/WFC undersamples the PSF by a factor of 2) improves the precision of individual photometry measurements and can reduce the impact of crowding. Together, these improvements reduce the uncertainty for NGC 3370 (see Table 13) by 50\% over other WFPC2-based calibrations.

The calibrations of photometric zero points for each camera are expected to be of similar quality. Each depends on either knowledge of the wavelength-dependent throughput or empirically determined color terms. However, the uncertainties in the zero points of each camera are relatively independent, so additional calibrations from ACS should reduce the overall systematic zero-point uncertainty.

Yet, the biggest advantage of ACS is the extension in distance over WFPC2 to which Cepheids can be measured in an economical amount of observing time. This added distance increases the sparse sample of SNe Ia whose luminosities can be calibrated with HST and improves the quality of the choices.

We expect the largest source of uncertainty to now be (if not previously) the distance estimate to the LMC. However, further work on the geometric maser distance to NGC 4258 (Herrnstein et al. 1999) and forthcoming observations of its Cepheids with ACS may remove this uncertainty as well. An added advantage of NGC 4258 (besides its accurate distance) is the similarity of its metallicity to the hosts of the ideal SN Ia calibrators, reducing the reliance on the accuracy of the metallicity corrections.

Here we have realized a significant improvement by updating the calibration of the Hubble constant from SNe Ia by using recent $\mathrm{SNe}$ Ia and a new HST camera. To continue to make progress in this field, the trend of replacing problematic data with modern data should continue. As this occurs, we expect the controversy over the value of $H_{0}$ as determined from $\mathrm{SNe}$ Ia to gradually abate. If the disagreement over the value of $H_{0}$ by groups using the same technique and the same data is taken as a measure of the uncertainty of its value, we believe that uncertainty can be reduced by a factor of 2 or more in the near future.

We wish to thank the Hubble Heritage team for help in producing a beautiful image of NGC 3370. Financial support for this work was provided by NASA through program GO9353 from the Space Telescope Science Institute, which is operated by AURA, Inc., under NASA contract NAS 5-26555. Additional funding was provided by the National Science Foundation for support of supernova research at Harvard University (AST 02-05808) and at the University of California, Berkeley (AST 03-07894). The Berkeley Automatic Imaging Telescope at Leuschner Observatory was funded by the NSF (AST 89-57063) and the University of California. Some of the data presented herein were obtained at the W. M. Keck Observatory, which is operated as a scientific partnership among the California Institute of Technology, the University of California, and NASA; the Observatory was made possible by the generous financial support of the W. M. Keck Foundation. A. V. F. is grateful for a Miller Research Professorship at the University of California, Berkeley, during which part of this work was completed.
Ayani, K., \& Yamaoka, H. 1998, IAU Circ., 6878, 1

Bennett, C., et al. 2003, ApJS, 148, 1

Boisseau, J. R., \& Wheeler, J. C. 1991, AJ, 101, 1281

Branch, D., Fisher, A., \& Nugent, P. 1993, AJ, 106, 2383

Branch, D., et al. 2003, AJ, 126, 1489

Buta, R., \& Turner, A. 1983, PASP, 95, 72

Cardelli, J. A., Clayton, G. C., \& Mathis, J. S. 1989, ApJ, 345, 245

de Vaucouleurs, G., de Vaucouleurs, A., Corwin, H. G., Jr., Buta, R. J., Paturel,

G., \& Fouqué, R. 1991, Third Reference Catalogue of Bright Galaxies (New

York: Springer)

Dolphin, A. E. 2000, PASP, 112, 1383

Ferrarese, L., et al. 2000, PASP, 112, 177

Filippenko, A. V. 1997, ARA\&A, 35, 309

\section{REFERENCES}

Freedman, W., et al. 1994, ApJ, 427, 628 2001, ApJ, 553, 47 (F01)

Fruchter, A., \& Hook, R. 1997, Proc. SPIE, 3164, 120

Garcia, A. M. 1993, A\&AS, 100, 47

Gibson, B. K., et al. 2000, ApJ, 529, 723

Gilliland, R., et al. 2004, STScI ACS Instrument Science Rep. 2004-01

Hamuy, M., Phillips, M. M., Maza, J., Suntzeff, N. B., Schommer, R. A., \& Aviles, R. 1995, AJ, 109, 1 1996a, AJ, 112, 2398

1996b, AJ, 112, 2408

Hatano, K., Branch, D., \& Deaton, J. 1998, ApJ, 502, 177

Herrnstein, J. R., et al. 1999, Nature, 400, 539

Hill, R. J., et al., 1998, ApJ, 496, 648 
Ho, W. C. G., Van Dyk, S. D., Peng, C. Y., Filippenko, A. V., Leonard, D. C., Matheson, T., Treffers, R. R., \& Richmond, M. W. 2001, PASP, 113, 1349

Holtzman, J. A., Burrows, C. J., Casertano, S., Hester, J. J., Trauger, J. T., Watson, A. M., \& Worthey, G. 1995, PASP, 107, 1065

Hurst, G. M., Armstrong, M., \& Arbour, R. 1998, IAU Circ., 6875, 1

Iijima, T., Cappellaro, E., \& Turatto, M. 1994, IAU Circ., 6108, 1

Jha, S. 2002, Ph.D. thesis, Harvard Univ.

Jha, S., et al. 1999, ApJS, 125, 73 2005, AJ, in press

Kennicutt, R., Jr., et al. 1998, ApJ, 498, 181

Kirshner, R. P., Willner, S. P., Becklin, E. E., Neugebauer, G., \& Oke, J. B. 1973, ApJ, 180, L97

Landolt, A. 1992, AJ, 104, 340

Leibundgut, B. 2001, ARA\&A, 39, 67

Leonard, D. C., Kanbur, S. M., Ngeow, C. C., \& Tanvir, N. R. 2003, ApJ, 594, 247

Lira, P. 1996, Master's thesis, Univ. Chile

Lira, P., et al. 1998, AJ, 115, 234 (erratum 116, 1006)

Madore, B. F. 1982, ApJ, 253, 575

Madore, B. F., \& Freedman, W. L. 1991, PASP, 103, 933 (MF91)

Oke, J. B., et al. 1995, PASP, 107, 375

Osterbrock, D. E. 1989, Astrophysics of Gaseous Nebulae and Active Galactic

Nuclei (Mill Valley: Univ. Science Books)

Parodi, B. R., et al. 2000, ApJ, 540, 634

Patat, F., Barbon, R., Cappellaro, E., \& Turatto, M. 1997, A\&A, 317, 423

Perlmutter, S., et al. 1997, ApJ, 483, 565

$$
\text { 1999, ApJ, 517, } 565
$$

Phillips, M. M. 1993, ApJ, 413, L105

Phillips, M. M., et al. 1999, AJ, 118, 1766

Richmond, M. W., Treffers, R. R., \& Filippenko, A. V. 1993, PASP, 105, 1164

Riess, A. G., \& Mack, 2004, STScI ACS Instrument Science Rep. 2004-06

Riess, A. G., Press, W. H., \& Kirshner, R. P. 1995, ApJ, 438, L17 1996a, ApJ, 473, 88 1996b, ApJ, 473, 588

Riess, A. G., et al. 1998, AJ, 116, 1009 1999a, AJ, 117, 707

1999b, AJ, 118, 2675 2004, ApJ, 607, 665

Rowan-Robinson, M. 2002, MNRAS, 332, 352

Saha, A., et al. 1996, ApJ, 466, 55
Saha, A., et al. 1997, ApJ, 486, 1 2001, ApJ, 562, 314

Sakai, S., Ferrarese, L., Kennicutt, R. C., Jr., \& Saha, A. 2004, ApJ, 608, 42 Schaefer, B. E. 1994, ApJ, 426, 493 . 1995, ApJ, 447, L13 1996, ApJ, 460, L19

Sebo, K. M., et al. 2002, ApJS, 142, 71

Sirianni, M., et al. 2005, PASP, in press

Spergel, D. N., et al. 2003, ApJS, 148, 175

Stetson, P. B. 1987, PASP, 99, 191 1993, in Calibrating the Hubble Space Telescope, ed. J. C. Blades \&

S. J. Osmer (Baltimore: STScI), 89 2000, PASP, 112, 925

Stetson, P. B., \& Gibson, B. K. 2001, MNRAS, 328, L1

Stetson, P. B., et al. 1998, ApJ, 508, 491

Suntzeff, N., et al. 1999, AJ, 117, 1175

Tammann, G. A., \& Reindl, B. 2002, Ap\&SS, 280, 165

Tanvir, N. R. 1997, in The Extragalactic Distance Scale, ed. M. Livio (Cambridge: Cambridge Univ. Press), 91

Thim, F., Tammann, G. A., Saha, A., Dolphin, A., Sandage, A., Tolstoy, E., \& Labhardt, L. 2003, ApJ, 590, 256

Tonry, J. L., et al. 2003, ApJ, 594, 1

Treffers, R. R., Filippenko, A. V., Van Dyk, S. D., Paik, Y., \& Richmond, M. W. 1995, in ASP Conf. Ser. 79, Robotic Telescopes: Current Capabilities, Present Developments, and Future Prospects for Automatic Astronomy, ed. G. W. Henry \& J. A. Eaton (San Francisco: ASP), 86

Treffers, R. R., Richmond, M. W., \& Filippenko, A. V. 1992, in ASP Conf. Ser. 34, Robotic Telescopes in the 1990s, ed. A. V. Filippenko (San Francisco: ASP), 115

Tripp, R., \& Branch, D. 1999, ApJ, 525, 209

Tsvetkov, D. Yu., \& Pavlyuk, N. N. 1997, Astron. Lett., 23, 26

Udalski, A., Szymanski, M., Kubiak, M., Pietrzynski, G., Soszynski, I., Wozniak, P., \& Zebrun, K. 1999, Acta Astron., 49, 223

Van Dyk, S. D., Treffers, R. R., Richmond, M. W., Filippenko, A. V., \& Paik, Y. B. 1994, IAU Circ., 6105, 1

Welch, D. L., \& Stetson, P. B. 1993, AJ, 105, 1813

Whitmore, B., Heyer, I., \& Casertano, S. 1999, PASP, 111, 1559

Zaritsky, D., Kennicutt, R. C., Jr., \& Huchra, J. P. 1994, ApJ, 420, 87

Zehavi, I., Riess, A. G., Kirshner, R. P., \& Dekel, A. 1998, ApJ, 503, 483 